\title{
A COMPARISON OF THE SERIES OF FOURIER AND BIRKHOFF*
}

\author{
BY
}

M. H. STONE

In his thesis, Haar has made a comparative study of developments on $(0, \pi)$ in terms of the set $\sqrt{1 / \pi}, \sqrt{2 / \pi} \cos x, \sqrt{2 / \pi} \cos 2 x, \cdots$, and of a certain Sturm-Liouville set. He proves that the term-by-term difference of the formal series for any function summable with summable square converges uniformly to zero on the interval $(0, \pi) \dagger$. Recently, Walsh, by entirely different and elegant methods, deduced a similar theorem for the set $\sqrt{2 / \pi} \sin x$, $\sqrt{2 / \pi} \sin 2 x, \cdots$, and a second Sturm-Liouville set on the interval $(0$, $\pi) \ddagger$. These two papers naturally suggested the possibility of analogous theorems for the series which Birkhoff defined in a memoir on the expansion problems of linear homogeneous differential systems of the $n$th order, and which are generalisations of the Sturm-Liouville series.§ Tamarkin, in a paper written to supplement the one just cited, compared the general Birkhoff series on the one hand and Fourier series on the other.\| He discussed only Riemann integrable functions and did not investigate the actual term-by-term difference of the series under consideration. In a later work on expansion problems which came to our attention after the completion of the present paper, he obtained, by methods different from our own. certain of our theorems, which will be noted subsequently. I Insofar a: Tamarkin's treatise concerns us here, it consists in an extension of his previous results to the case of the summable function.

In the present paper we study the comparative properties not only of the Birkhoff and Fourier series for an arbitrary summable function, but also of the formal series obtained by deriving these term-by-term $k$ times.

* Presented to the Society, December 30, 1924; accepted in partial fulfillment of the requirements for the degree of Doctor of Philosophy at Harvard University; received by the editors of these Transactions in October, 1925.

† Haar, Mathematische Annalen, vol. 69 (1910), pp. 331-371.

$\ddagger$ Walsh, Annals of Mathematics, ser. 2, vol. 24, pp. 109-120.

\$ Birkhoff, these Transactions, vol. 9( 1908), pp. 373-395.

|| Tamarkin, Rendiconti del Circolo Matematico di Palermo, vol. 34 (1912), pp. 345-395.

IT Tamarkin, OnCertain General Problems in the Theory of Ordinary Linear Differential Equations and the Expansion of an Arbitrary Function in Series, Petrograd, 1917, in Russian: cited henceforth as D. E. 
While our main purpose is the investigation of these questions, we first make generalisations of the series defined in Birkhoff's memoir. These generalisations are not trivial, and furthermore occupy a central position in the theory of linear differential equations. For this reason we take the liberty of describing them here.

\section{EXISTENCE THEOREMS}

In the following pages we shall have constantly before us the differential equation

$$
u^{(n)}+\star+p_{2} u^{(n-2)}+\cdots+p_{n-1} u^{(1)}+\left(p_{n}+\lambda\right) u=0
$$

where $p_{2}, \cdots, p_{n}$ are real or complex summable functions of the real variable $x$ on the interval $(0,1)$, and $\lambda$ is a complex parameter. The usual existence theorems are obviously inapplicable to the present case. We employ a modification of the method of successive approximations to show the existence of "solutions" of such equations.* We first prove

Theorem I. If

(1) $p_{1}, \cdots, p_{n}, r$ are real or complex summable functions of the real variable $x, a \leqq x \leqq b$;

(2) $\lambda$ is a complex parameter restricted to the circle $|\lambda| \leqq \Lambda$;

(3) $x$ is any point of $(a, b)$;

(4) $c_{0}, \cdots, c_{n-1}$ are any real or complex constants;

then there exists a constant $\delta_{\Lambda}>0$, independent of $\lambda, x_{0}, c_{0}, \cdots, c_{n-1}$; and there exists a function $u(x, \lambda)$, defined for all $\lambda,|\lambda| \leqq \Lambda$, and for all $x$ on the interval common to $(a, b)$ and $\left(x_{0}-\delta_{\Lambda}, x_{0}+\delta_{\Lambda}\right)$, such that

(1) $u^{(k)}(x, \lambda)$ is continuous in $x$ and analytic in $\lambda, k=0, \cdots, n-1$, the differentiation being with respect to $x$;

(2) $u^{(k)}\left(x_{0}, \lambda\right)=c_{k}, k=0, \cdots, n-1$;

(3) $u^{(n)}(x, \lambda)$ is summable in $x$ for each value of $\lambda$;

(4) $u^{(n-1)}(x, \lambda)=c_{n-1}+\int_{x_{0}}^{x} u^{(n)}(x, \lambda) d x$;

(5) $u^{(n)}+p_{1} u^{(n-1)}+\cdots+p_{n-1} u^{(1)}+\left(p_{n}+\lambda\right)=r$, except possibly on a set of zero measure, $E_{\lambda}$, for each $\lambda$;

(6) $u(x, \lambda)$ is unique.

In the case $p_{1}=p_{2}=\cdots=p_{n}=0, \Lambda=0$, the equation reduces to $u^{(n)}=r$; and we can construct a function with the properties (1)-(5) of the theorem for the whole interval $(a, b)$. By direct verification we see that it is

$$
u=c_{0}+\frac{c_{1}\left(x-x_{0}\right)}{2}+\cdots+\frac{c_{n-1}\left(x-x_{0}\right)^{n-1}}{(n-1) !}+\int_{x_{0}}^{x} \frac{(x-\xi)^{n-1}}{(n-1) !} r(\xi) d \xi .
$$

* Tamarkin has obtained existence theorems here differently, D. E., Chapter I. 
Hence in the general case we attempt to solve the equation

$$
\begin{gathered}
u=c_{0}+\frac{c_{1}\left(x-x_{0}\right)}{2}+\cdots+\frac{c_{n-1}\left(x-x_{0}\right)^{n-1}}{(n-1) !}+\int_{x_{0}}^{x} \frac{(x-\xi)^{n-1}}{(n-1) !}[S(u)+r] d \xi, \\
S(u)=-p_{1} u^{(n-1)}-\cdots-p_{n-1} u^{(1)}-\left(p_{n}+\lambda\right) u .
\end{gathered}
$$

We first form the sequence of successive approximants $\left[u_{i}\right]$ where

$$
\begin{aligned}
u_{0} & =c_{0}+\frac{c_{1}\left(x-x_{0}\right)}{2}+\cdots+\frac{c_{n-1}\left(x-x_{0}\right)^{n-1}}{(n-1) !}, \\
u_{i+1} & =u_{0}+\int_{x_{0}}^{x} \frac{(x-\xi)^{n-1}}{(n-1) !}\left[S\left(u_{i}\right)+r(\xi)\right] d \xi .
\end{aligned}
$$

To consider the convergence of this sequence we construct a second sequence where

$$
\begin{aligned}
v_{0} & =u_{0}, \\
v_{1} & =u_{1}-u_{0}=\int_{x_{0}}^{x} \frac{(x-\xi)^{n-1}}{(n-1) !}\left[S\left(u_{0}\right)+r\right] d \xi \\
v_{i+1} & =u_{i+1}-u_{i}=\int_{x_{0}}^{x(x-\xi)^{n-1}}-S\left(v_{i}\right) d \xi \quad(i=1,2, \cdots) .
\end{aligned}
$$

Clearly, for $k=0,1,2, \cdots, n-1$ and $i=1,2, \cdots$, and for all $x, x_{0}, \xi$ on $(a, b)$ we have

and

$$
\left|v_{1}{ }^{(k)}\right| \leqq C, \quad\left|\frac{(x-\xi)^{(n-k-1)}}{(n-k-1) !}\right| \leqq L,
$$

$$
v_{i+1}^{(k)}=\int_{x_{0}}^{x} \frac{(x-\xi)^{n-k-1}}{(n-k-1) !} S\left(v_{i}\right) d \xi .
$$

Next, by a fundamental property of the Lebesgue integral, we can write

$$
\left|\int_{x_{0}}^{x}\left(\left|p_{1}\right|+\cdots+\left|p_{n}\right|+|\lambda|\right) d \xi\right| \leqq \frac{1}{2 L}
$$

for all $\lambda,|\lambda| \leqq \Lambda$, and for all $x,\left|x-x_{0}\right| \leqq \delta_{\Lambda}$, where $\delta_{\Lambda}>0$ does not depend on $x_{0}$. We now show that if the inequality $\left|v_{i}{ }^{(k)}\right| \leqq C / 2^{i-1}$ is true, $\left|x-x_{0}\right| \leqq \delta_{\Lambda}$, it is also true when $i$,is replaced by $i+1$; since it holds for $i=1$, it will be true for all $i$. We have

$$
\begin{array}{r}
\left|v_{i+1}^{(k)}\right| \leqq L\left|\int_{x_{0}}^{x}\left(\left|p_{1}\right|+\cdots+\left|p_{n}\right|+|\lambda|\right) d \xi\right| \frac{C}{2^{i-1}} \leqq \frac{C}{2^{i}}, \\
\left|x-x_{0}\right| \leqq \delta_{\Lambda} .
\end{array}
$$


Hence the infinite series $v_{0}^{(k)}+v_{1}^{(k)}+v_{2}^{(k)}+\cdots, k=0, \cdots, n-1$, converge uniformly, $\left|x-x_{0}\right| \leqq \delta_{\Lambda},|\lambda| \leqq \Lambda$. The individual terms are continuous in $x$ and polynomial in $\lambda$. We can thus be sure that the limit functions are continuous in $x$ and analytic in $\lambda$, and can write

$$
\lim _{i \rightarrow \infty} \sum_{\alpha=1}^{\alpha=i} v_{\alpha}=\lim _{i \rightarrow \infty} u_{i}^{(k)}=u^{(k)}(x, \lambda) .
$$

The functions $u^{(k)}(x, \lambda)$ therefore satisfy conclusion (1) of our theorem. On allowing $i$ to become infinite in the relation connecting $u_{i+1}$ and $u_{i}$, we find

$$
u(x, \lambda)=u_{0}(x)+\int_{x_{0}}^{x} \frac{(x-\xi)^{n-1}}{(n-1) !}[S(u)+r] d \xi, \quad\left|x-x_{0}\right| \leqq \delta_{\Lambda} .
$$

By direct computation we see that (2) is fulfilled. On deriving the identity $n$ times with respect to $x$ there results

$$
u^{(n)}(x, \lambda)=S(u)+r(x)
$$

except possibly on a set $E_{\lambda}$ of measure zero. Thus (3) and (5) are satisfied. Then we see that

$$
\begin{aligned}
u^{(n-1)}(x, \lambda) & =c_{n-1}+\int_{x_{0}}^{x}[S(u)+r] d \xi \\
& =c_{n-1}+\int_{x_{0}}^{x} u^{(n)}(x, \lambda) d x
\end{aligned}
$$

which is conclusion (4).

We now assume that on some interval containing $x$ there exists a second lunction $\bar{u}(x, \lambda),|\lambda| \leqq \Lambda$, with the properties

(1') $\quad \bar{u}^{(k)}(x, \lambda), k=0, \cdots, n-1$, is continuous in $x$ for each value of $\lambda$;

$\left(2^{\prime}\right) \quad \bar{u}^{(k)}\left(x_{0}, \lambda\right)=c_{k}, k=0, \cdots, n-1$;

$\left(3^{\prime}\right) \quad \bar{u}^{(n)}(x, \lambda)$ is summable in $x$ for each value of $\lambda$;

$\left(4^{\prime}\right) \quad \bar{u}^{(n-1)}(x, \lambda)=c_{n-1}+\int_{x_{0}}^{x} \bar{u}^{(n)}(x, \lambda) d x$;

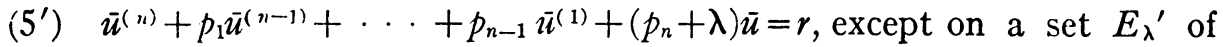
zero measure.

The difference $U=u-\bar{u}$ is then a function satisfying the conditions $\left(1^{\prime}\right)$ $-\left(5^{\prime}\right)$ with $c_{k}=0, k=0, \cdots, n-1$, and $r(x) \equiv 0$, the set $E_{\lambda}{ }^{\prime}$ being replaced by the set $E_{\lambda}{ }^{\prime \prime}=E_{\lambda}+E_{\lambda}{ }^{\prime}$. We suppose that at some point $\left(\bar{x}_{0}, \lambda_{0}\right)$ in the domain of definition of $U(x, \lambda)$ we have $U\left(\bar{x}_{0}, \lambda_{0}\right) \neq 0$.

By the part of the theorem already proved we now construct functions $U_{2}, \cdots, U_{n}$, satisfying the conditions (1)-(5) with $U_{i}^{(k)}\left(\bar{x}_{0}, \lambda\right)=\delta_{i}, k+1$, the well known Kronecker symbol, and $r(x) \equiv 0$. We then form 


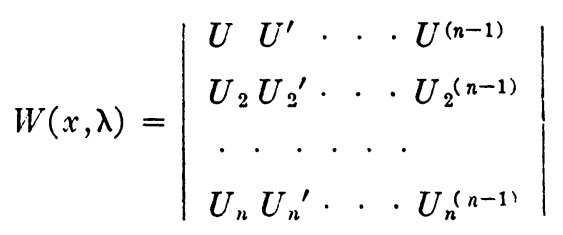

with $W\left(x_{0}, \lambda\right)=0, W\left(\bar{x}_{0}, \lambda\right)=U^{r}\left(\bar{x}_{0}, \lambda\right) \neq 0$. It is eavily seen by the use of (5) and $\left(5^{\prime}\right)$ that we have

$$
W^{\prime}(x, \lambda)=-p_{1}(x) W(x, \lambda)
$$

almost everywhere, while by (3) and $\left(3^{\prime}\right)$ it is clear that

$$
W(x, \lambda)=\int_{x_{0}}^{x} W^{\prime}(x, \lambda) d x .
$$

On the other hand the function

satisfies

$$
W_{0}(x)=e^{-\int_{x_{0}}^{x} P_{d} d x}
$$

$$
W_{0}^{\prime}(x)=-p_{1}(x) W_{0}(x)
$$

almost everywhere, and

$$
W_{0}(x)=1+\int_{x_{0}}^{x} W_{0}^{\prime}(x) d x .
$$

We then find that $\left(W / W_{0}\right)^{\prime}=0$ almost everywhere; and we can interrate this relation obtaining $W=c(\lambda) W_{0}$. Since $W\left(x_{0}, \lambda\right)=0$, it follows that $c(\lambda)=0, W(x, \lambda) \equiv 0$. This leads to the contradictory statement $W\left(\bar{x}_{0}, \lambda\right)$ $=U\left(\bar{x}_{0}, \lambda\right)=0$. We thus have $U(x, \lambda) \equiv 0$ over the domain for which it is defined; in other words, $u(x, \lambda)$ is unique, as asserted in the sixth point of the theorem.

It is now easy to demonstrate the general existence theorem, which is

THEOREM II. Under the hypotheses (1)-(4) of Theorem I ihere exists a unique function $u(x, \lambda), a \leqq x \leqq b$, such that

(1) $u^{(k)}(x, \lambda)$ is continuous in $x$ and entire in $\lambda, k=0, \cdots, n-1$;

(2) $u^{(k)}\left(x_{0}, \lambda\right)=c_{k}, k=0, \cdots, n-1$;

(3) $u^{(n)}(x, \lambda)$ is summable in $x$ for each value of $\lambda$;

(4) $u^{(n-1)}(x, \lambda)=c_{n-1}+\int_{x_{0}}^{x} u^{(n)}(x, \lambda) d x$;

(5) $u^{(n)}+p_{1} u^{(n-1)}+\cdots+p_{n-1} u^{(1)}+\left(p_{n}+\lambda\right) u=r$, except possibly on a set $\varepsilon$ of measure zero, independent of $\lambda$.

At $x_{0}$ we form the interval $I_{0}:\left|x-x_{0}\right| \leqq \delta_{\Lambda}$; at the right-hand end of $I_{0}$, $x_{0}+\delta_{\Lambda}$, we form the interval $I_{1}:\left|x-x_{0}-\delta_{\Lambda}\right| \leqq \delta_{\Lambda}$; and we continue, forming 
$I_{2}, \cdots, I_{N}$, where $N$ is large enough that $I_{N}$ contains $x=b$. Similarly, we form a chain of intervals $I_{-1}, \cdots, I_{-M}$ running to the left, with $M$ large enough that $I_{-}$contains $x=a$. Then in $I_{0}$ we use Theorem $\mathrm{I}$ to set up a function $u(x, \lambda)$ satisfying conditions $(1)-(6)$ of that theorem. In $I_{1}$ we apply Theorem I to define a function $u_{1}(x, \lambda)$ satisfying (1)-(6) in $I_{1}$, with $u_{1}^{(k)}\left(x_{0}+\delta_{\Lambda}, \lambda\right)=u^{(k)}\left(x_{0}+\delta_{\Lambda}, \lambda\right)$; by (6), $u$ and $u_{1}$ coincide, $x_{0} \leqq x \leqq x_{0}+\delta_{\Lambda}$, $|\lambda| \leqq \Lambda$. We then perform a similar set of operations in $I_{2}, \cdots, I_{N}, I_{-1}, \cdots$, $I_{-M}$. The result is the formation of the function $u(x, \lambda)$ whose existence is asserted in the present theorem.

For this function properties (1)-(4) are immediate. In order to prove (5) we start from the fact that for each $\lambda$ the differential equation is satisfied except on a set $E_{\lambda}$ of zero measure. We denote by $\lambda^{\prime}$ the points of the $\lambda$-plane with rational coördinates. We then define the set of zero measure

Writing

$$
\varepsilon_{1}=\sum_{\left(\lambda^{\prime}\right)} E_{\lambda}^{\prime} .
$$

$$
P(x)=\int_{x_{0}}^{x}\left(\left|p_{1}\right|+\cdots+\left|p_{n}\right|+1\right) d x,
$$

we denote by $\varepsilon_{2}$ the set of zero measure on which $P^{\prime}$ does not exist, is infinite or does not coincide with the integrand in the definition of $P$. We show that we can set $\varepsilon=\varepsilon_{1}+\varepsilon_{2}$. We let $x^{\prime}$ be any point of $(a, b)$ not in $\varepsilon, \lambda$ any point of the complex plane, and $\lambda^{\prime}$ a point sufficiently close to $\lambda$ that for all $x$ on $(a, b)$ and for $k=0, \cdots, n-1$, the inequalities

$$
\left|u^{(k)}(x, \lambda)-u^{(k)}\left(x, \lambda^{\prime}\right)\right|<\epsilon, \quad\left|\lambda u(x, \lambda)-\lambda^{\prime} u\left(x, \lambda^{\prime}\right)\right|<\epsilon
$$

are true. We find

$$
\begin{aligned}
& \left.\left.\underset{h \rightarrow 0}{\lim \sup } \mid \frac{1}{h} u^{(n-1)}(x, \lambda)\right]_{x=x^{\prime}}^{x=x^{\prime}+h}-\frac{1}{h} u^{(n-1)}\left(x, \lambda^{\prime}\right)\right]_{x=x^{\prime}}^{x=x^{\prime}+h} \mid \\
& \left.\left.=\limsup _{h \rightarrow 0} \mid \frac{1}{h} \int_{x^{\prime}}^{x^{\prime}+h}\left\{p_{1} u^{(n-1)}\right]_{\lambda}^{\lambda^{\prime}}+\cdots+p^{n} u\right]_{\lambda}^{\lambda^{\prime}}+\lambda^{\prime} u\left(x, \lambda^{\prime}\right)-\lambda u(x, \lambda)\right\} d x \mid \\
& \leqq \epsilon \lim _{h \rightarrow 0} \frac{P\left(x^{\prime}+h\right)-P\left(x^{\prime}\right)}{h}=\epsilon P^{\prime}\left(x^{\prime}\right) .
\end{aligned}
$$

Since

$$
\left.\lim _{h \rightarrow 0} \frac{1}{h} u^{(n-1)}\left(x, \lambda^{\prime}\right)\right]_{x=x^{\prime}}^{x=x^{\prime}+h}=-p_{1} u^{(n-1)}-\cdots-p_{n} u-\lambda^{\prime} u+r,
$$

and since $\epsilon$ can be taken arbitrarily small, we must have

$$
\left.\lim _{h \rightarrow \infty} \frac{1}{h} u^{(n-1)}(x, \lambda)\right]_{x=x^{\prime}}^{x=x^{\prime}+h}=-b_{1} u^{(n-1)}-\cdots-p_{n} u-\lambda u+r,
$$

as we were to show. 
All the usual discussions of linear dependence and independence of solutions, solutions satisfying given boundary conditions, Green's function, and so forth, can now be repeated for the general equation described in the theorem.* We may note that a consideration of the linear dependence of solutions would lead to a sharpening of Theorem II in that the set $\varepsilon$ could be replaced by a set $E$, independent of the particular solution.

We turn next to another type of theorem. While that which we shall now discuss differs but slightly from a less general theorem of Birkhoff in its proof, we take the opportunity of giving a complete demonstration since the details of the proof can be simplified for our purposes. $\dagger$

We denote by $S$ a sector of the type $l \pi / n \leqq \theta \leqq(l+1) \pi / n, \arg \rho=\theta$, where $l=0,1,2, \cdots, 2 n-1$. From the sector $S$ we define a region $T$ by an arbitrary translation taking the vertex into the point $-c$, where $c$ is a complex constant. The $n$ distinct roots of $\omega^{n}+1=0$ can be written in the order $\omega_{1}, \cdots, \omega_{n}$ such that for all $\rho$ on $T$

$$
R\left(\overline{\rho+c} \omega_{1}\right) \leqq R\left(\overline{\rho+c} \omega_{2}\right) \leqq \cdots \leqq R\left(\overline{\rho+c} \omega_{n}\right)
$$

where the notation $R($ ) means, as usual, "the real part of". $\ddagger$ We commence with two important lemmas. The first is

LEMMA I. On the region $T$ of the $\rho$-plane

$$
\begin{aligned}
\left|\frac{d^{k}}{d x^{k}} \sum_{\alpha=1}^{\alpha=i} \omega_{\alpha} e^{\rho \omega_{\alpha}(x-\xi)}\right| & \leqq C|\rho|^{k} i\left|e^{\rho \omega_{i}(x-\xi)}\right|, & & 0 \leqq \xi \leqq x \leqq 1, \\
\left|\frac{d^{k}}{d x^{k}} \sum_{\alpha=i+1}^{\alpha=n} \omega_{\alpha} e^{\rho \omega_{\alpha}(x-\xi)}\right| & \leqq C|\rho|^{k}(n-i)\left|e^{\rho \omega_{i}(x-\xi)}\right|, & & 0 \leqq x \leqq \xi \leqq 1,
\end{aligned}
$$

for $k=0, \cdots, n-1, i=1, \cdots, n$. The constant $C$ may be taken independent of $i$ and $k$.

We let $C$ be chosen so that

$$
\begin{aligned}
& \left|e^{c\left(\omega_{i}-\omega_{\alpha}\right)(x-\xi)}\right| \leqq C, \alpha=1, \cdots, i, i=1, \cdots, n, 0 \leqq \xi \leqq x \leqq 1 \\
& \left|e^{c\left(\omega_{i}-\omega_{\alpha}\right)(x-\xi)}\right| \leqq C, \alpha=i+1, \cdots, n, i=1, \cdots, n, 0 \leqq x \leqq \xi \leqq 1 .
\end{aligned}
$$

Then we have for $\alpha \leqq i$ and for $\rho$ on $T$

$$
R\left(\rho \omega_{\alpha}\right) \leqq R\left(\rho \omega_{i}+\overline{c \omega_{i}-\omega_{\alpha}}\right) .
$$

* For the usual existence theorems and subsequent developments, see Bôcher, Leçons sur les Methodes de Sturm, Paris, 1917.

$\dagger$ Birkhoff, these Transactions, vol. 9 (1908), pp. 219-231.

$\ddagger$ Birkhoff, these Transactions, vol. 9 (1908), p. 381. 
Thus, if $x \geqq \xi$,

$$
\begin{aligned}
\left|\frac{d^{k}}{d x^{k}} \sum_{\alpha=1}^{\alpha=i} \omega_{\alpha} e^{\rho \omega_{\alpha}(x-\xi)}\right| & \leqq|\rho|^{k} \sum_{\alpha=1}^{\alpha=i}\left|e^{\rho \omega_{\alpha}(x-\xi)}\right| \leqq|\rho|^{k} \sum_{\alpha=1}^{\alpha=i}\left|e^{\left(\rho \omega_{i}+c \overline{\omega_{i}-\omega_{\alpha}}\right)(x-\xi)}\right| \\
& \leqq C|\rho|^{k} i\left|e^{\rho \omega_{i}(x-\xi)}\right|, \quad 0 \leqq \xi \leqq x \leqq 1,
\end{aligned}
$$

as we were to show.

Again, starting from the inequality $R\left(\rho \omega_{\alpha}\right) \geqq R\left(\rho \omega_{i}+c \overline{\omega_{i}-\omega_{\alpha}}\right), \alpha \geqq i$, which is true on $T$, we derive the second inequality by analogous means.

In Lemma II we shall employ heavy-faced type to denote square matrices of $n^{2}$ elements. ${ }^{*}$ The statement of the lemma is as follows:

LEMMA II. If $\boldsymbol{\Omega}(\boldsymbol{x})$ has all its elements real or complex continuous functions of $x, 0 \leqq x \leqq 1$, and if $K(x, \xi, \rho)$ has all its elements of the form $p(\xi) k(x, \xi, \rho)$ where

(1) $p(\xi)$ is a real or complex summable function, $0 \leqq \xi \leqq 1$;

(2) $k(x, \xi, \rho)$ is continuous in $x$ and $\xi$ for $0 \leqq \xi<x \leqq 1$ and for $0 \leqq x<\xi \leqq 1$, and is analytic in $\rho,|\rho| \geqq R$ on $T$;

(3) $|k(x, \xi, \rho)| \leqq k, 0 \leqq x \leqq 1,0 \leqq \xi \leqq 1,|\rho| \geqq R$ on $T$;

then there exists a matrix $Z(x, \rho), 0 \leqq x \leqq 1,|\rho|$ sufficiently large on $T$, whose elements are continuous in $x$ and analytic in $\rho$, and which satisfies

$$
Z(x, \rho)=\Omega(x)+\frac{1}{\rho} \int_{0}^{1} Z(\xi, \rho) K(x, \xi, \rho) d \xi .
$$

Furthermore,

$$
\boldsymbol{Z}(x, \rho)=\boldsymbol{\Omega}(x)+\frac{1}{\rho} E(x, \rho) \text { where }|E(x, \rho)| \leqq M, 0 \leqq x \leqq 1,
$$

$\rho$ on $T$.

If $U$ denotes the matrix all of whose elements are unity, we can write

$$
|\mathbf{\Omega}| \leqq \omega U, \int_{0}^{1}|K(x, \xi, \rho)| d \xi \leqq p U
$$

We consider the infinite series

$$
\begin{aligned}
Z(x, \rho)= & \mathbf{\Omega}(x)+ \\
& \sum_{k=1}^{\infty} \frac{1}{\rho^{k}} \int_{0}^{1} \cdots \int_{0}^{1} \Omega\left(\xi_{k-1}\right) K\left(\xi_{k-2}, \xi_{k-1}, \rho\right) \cdots K(x, \xi, \rho) d \xi_{k-1} \cdots d \xi .
\end{aligned}
$$

- For further details of notation see Birkhoffa nd Langer, Proceedings of the Ameri can Academy of Arts and Sciences, vol. 58 (1923), pp. 52-54. 
We see that the general term is in absolute value less than or equal to

$$
\frac{1}{|\rho|^{k}} \omega p^{k} U^{k+1}=\frac{n^{k+1} \omega p^{k}}{|\rho|^{k}} U \text {. }
$$

Thus if we take $n p /|\rho| \leqq \theta<1$, the series converges absolutely and uniformly in $x$ and $\rho$. The individual terms are continuous in $x$ and analytic in $\rho$; hence this is true of the elements of $\boldsymbol{Z}$. By direct computation with this series we find that

$$
Z(x, \rho)=\Omega(x)+\frac{1}{\rho} \int_{0}^{1} Z(\xi, \rho) K(x, \xi, \rho) d \xi .
$$

We now let $m(\rho)$ be the maximum value taken on by the elements of $\boldsymbol{Z}(x, \rho)$ for $0 \leqq x \leqq 1$. Then we have $|\boldsymbol{Z}(x, \rho)| \leqq m(\rho) U$. From the integral equation

$$
|Z(x, \rho)| \leqq \omega U+\frac{1}{|\rho|} p m(\rho) U^{2}=\left(\omega+\frac{p n m(\rho)}{|\rho|}\right) U .
$$

All the elements of the matrix on the right are equal; there is always some element of the matrix on the left which for an appropriate value of $x$ takes on the value $m(\rho)$. Hence

$$
m(\rho) \leqq \omega+\frac{n p m(\rho)}{|\rho|}, m(\rho) \leqq \frac{\omega}{1-\frac{p n}{|\rho|}} \leqq m, \quad|\rho| \geqq R^{\prime} .
$$

Now we find the inequality

$$
|Z(x, \rho)-\Omega(x)| \leqq \frac{1}{|\rho|} m(\rho) p U^{2} \leqq \frac{n m p}{|\rho|} U
$$

from which the rest of the lemma follows.

We come now to

THEOREM III. If $p_{2}, \cdots, p_{n}$ are real or complex summable functions of the real variable $x, 0 \leqq x \leqq 1$, and if $\rho$ is a complex parameter, then the differential equation $u^{(n)}+\star+p_{2} u^{(n-2)}+\cdots+p_{n-1} u^{(1)}+\left(p_{n}+\rho^{n}\right) u=0$ has on any region $T$ of the complex $\rho$-plane, $n$ linearly independent solutions analytic in $\rho, u_{1}, \cdots, u_{n}$, expressible with their first $n-1$ derivatives in the form

$$
u_{i}{ }^{(k)}(x, \rho)=\rho^{k} e^{\rho \omega_{i} x}\left(\omega_{i}^{k}+\frac{E_{i k}(x, \rho)}{\rho}\right)(i=1, \cdots, n ; k=0, \cdots, n-1),
$$

where $\left|E_{i k}(x, \rho)\right| \leqq M$ for all $\rho$ on $T,|\rho| \geqq R$, and for all $x, 0 \leqq x \leqq 1$. 
We write the differential equation as

$$
u^{(n)}+\rho^{n} u=-p_{2} u^{(n-2)}-\cdots-p_{n} u=-S^{\prime}(u),
$$

and proceed to solve this equation as though the right-hand side were known. Thus

$$
u=c_{1} e^{\rho \omega_{1} x}+\cdots+c_{n} e^{\rho \omega_{n} x}+\int_{0}^{x} \frac{\omega_{1} e^{\rho \omega_{1}(x-\xi)}+\cdots+\omega_{n} e^{\rho \omega_{n}(x-\xi)}}{n \rho^{n-1}} S^{\prime}(u) d \xi,
$$

where $c_{1}, \cdots, c_{n}$ are arbitrary constants. We now write

$$
\begin{array}{lrl}
\bar{c}_{l}=c_{l} & (l=1, \cdots, i), \\
\bar{c}_{l}=c_{l}+\sum_{\alpha=i+1}^{\alpha=n} \int_{0}^{1} \frac{\omega_{\alpha} e^{-\rho \omega_{\alpha} \xi}}{n \rho^{n-1}} S^{\prime}(u) d \xi & (l=i+1, \cdots, n) .
\end{array}
$$

We find at once

$$
\begin{aligned}
u=\bar{c}_{1} e^{\rho \omega_{1} x}+\cdots+\bar{c}_{n} e^{\rho \omega_{n} x} & +\frac{1}{n \rho^{n-1}} \int_{0}^{x} \sum_{\alpha=1}^{\alpha=1} \omega_{\alpha} e^{\rho \omega_{\alpha}(x-\xi)} S^{\prime}(u) d \xi \\
& +\frac{1}{n \rho^{n-1}} \int_{1}^{x} \sum_{\alpha=i+1}^{\alpha=n} \omega_{\alpha} e^{\rho \omega_{\alpha}(x-\xi)} S^{\prime}(u) d \xi .
\end{aligned}
$$

Next we let $u_{i}$ correspond to $\bar{c}_{1}=\cdots=\bar{c}_{i-1}=\bar{c}_{i+1}=\cdots=\bar{c}_{n}=0, \bar{c}_{i}=1$. We then put $u_{i}{ }^{(k)}=\rho^{k} e^{\rho \omega i x} z_{i k}$ for $i=1, \cdots, n$ and $k=0, \cdots, n-1$. Then it is apparent that

$$
\begin{aligned}
\rho^{k} e^{\rho \omega_{i} x_{Z_{i k}}=\omega_{i}^{k} \rho^{k} e^{\rho \omega_{i} x}} & +\frac{1}{n \rho^{n-1}} \int_{0}^{x}\left\{\frac{d^{k}}{d x^{k}} \sum_{\alpha=1}^{\alpha=1} \omega_{\alpha} e^{\rho \omega_{\alpha}(x-\xi)}\right\} S^{\prime}(u) d \xi \\
& +\frac{1}{n \rho^{n-1}} \int_{1}^{x}\left\{\frac{d^{k}}{d x^{k}} \sum_{\alpha=1}^{\alpha=n} \omega_{\alpha} e^{\rho \omega_{\alpha}(x-\xi)}\right\} S^{\prime}(u) d \xi .
\end{aligned}
$$

On expressing $S^{\prime}(u)$ in terms of the $z_{i k}$ this equation becomes

$$
\begin{array}{r}
z_{i k}=\omega_{i}^{k}+\frac{1}{n \rho} \int_{0}^{x} e^{-\rho \omega_{0}(x-\xi)} \rho^{-k}\left\{\frac{d^{k}}{d x^{k}} \sum_{\alpha=1}^{\alpha=i} \omega_{\alpha} e^{\rho \omega_{\alpha}(x-\xi)}\right\}\left\{p_{2} z_{i, n-2}+\right. \\
\left.\cdots+\frac{p_{n}}{\rho^{n-2}} z_{i, 0}\right\} d \xi \\
+\frac{1}{n \rho} \int_{1}^{x} e^{-\rho \omega_{i}(x-\xi)} \rho^{-k}\left\{\frac{d^{k}}{d x^{k}} \sum_{\alpha=i+1}^{\alpha=1} \omega_{\alpha} e^{\rho \omega_{\alpha}(x-\xi)}\right\} \\
\cdots p_{2} z_{i, n-2}+ \\
\left.\cdots+\frac{p_{n}}{\rho^{n-2}} z_{i, 0}\right\} d \xi .
\end{array}
$$


By Lemma I we see that this set of equations is precisely of the sort discussed in Lemma II. Thus we obtain solutions $u_{1}, \cdots, u_{n}$ such that $u_{i}^{(k)}(x, \rho)$ is continuous in $x, 0 \leqq x \leqq 1$, and analytic in $\rho,|\rho| \geqq R$, on $T$, for $i=1, \cdots$, $n, k=0, \cdots, n-1$, while

$$
u_{i}^{(k)}(x, \rho)=\rho^{k} e^{\rho \omega_{i x}}\left\{\omega_{i}^{k}+\frac{E_{i k}(x, \rho)}{\rho}\right\}, \quad E_{i k} \mid \leqq M .
$$

The functions $u_{1}, \cdots, u_{n}$ are clearly linearly independent for large $|\rho|$, by their asymptotic form.

It remains for us to show that the $u_{1}, \cdots, u_{n}$ satisfy the differential equation. We know that there exist solutions $\bar{u}_{1}, \ldots, \bar{u}_{n}$ of the differential equation, linearly independent for all values of $\rho$; we need only apply Theorem II taking $\bar{u}_{i}{ }^{(k)}(0)=\delta_{i, k+1}, i=1, \cdots, n, k=0, \cdots, n-1$. By assigning appropriate values, analytic in $\rho$, to the coefficients $\bar{c}_{1}, \ldots, \bar{c}_{n}$ of the integral equation above, we obtain particular equations satisfied by $\bar{u}_{1}, \cdots, \bar{u}_{n}$. We show that any solution $u$ for a similar equation in which $\bar{c}_{1}, \cdots, \bar{c}_{n}$ are constants can be expressed linearly in terms of $\bar{u}_{1}, \cdots, \bar{u}_{n}$ and is consequently a solution of the differential equation. We write

$$
\begin{aligned}
U=u-c_{1} \bar{u}_{1}-\cdots-c_{n} \bar{u}_{n}, & \\
U=A_{1} e^{\rho \omega_{1} x}+\cdots+A_{n} e^{\rho \omega_{n} x} & +\frac{1}{n \rho^{n-1}} \int_{0}^{x} \sum_{\alpha=1}^{\alpha=i} \omega_{\alpha} e^{\rho \omega_{\alpha}(x-\xi)} S^{\prime}(U) d \xi \\
& +\frac{1}{n \rho^{n-1}} \int_{1}^{x} \sum_{\alpha=i+1}^{\alpha=n} \omega_{\alpha} e^{\rho \omega_{\alpha}(x-\xi)} S^{\prime}(U) d \xi .
\end{aligned}
$$

We see now that either (1) when the constants $\bar{c}_{1}, \cdots, \bar{c}_{n}$ are not all zero, we can determine $A_{1}, \cdots, A_{n}$ identically zero by an appropriate choice of $C_{1}, \cdots, C_{n}$; or (2) we can take $u=0$ and determine $C_{1}, \cdots$, $C_{n}$ not all zero so that $A_{1}, \cdots, A_{n}$ are identically zero. Either possibility leads us to study the equation last written down with the coefficients $A_{1}$, $\cdots, A_{n}$ taken equal to zero. On writing $U^{(k)}=\rho^{k} e^{\rho \omega_{i x}} Z_{k}$ we find

$$
\begin{array}{r}
Z_{k}=\frac{1}{n \rho^{n-1}} \int_{0}^{x} \rho^{-k} e^{-\rho \omega_{i}(x-\xi)}\left\{\frac{d^{k}}{d x^{k}} \sum_{\alpha=1}^{\alpha=i} \omega_{\alpha} e^{\rho \omega_{\alpha}(x-\xi)}\right\}\left\{p_{2} \rho^{n-2} Z_{n-2}+\right. \\
\left.\cdots+p_{n} Z_{0}\right\} d \xi \\
+\frac{1}{n \rho^{n-1}} \int_{1}^{x} \rho^{-k} e^{-\rho \omega_{i}(x-\xi)}\left\{\frac{d^{k}}{d x^{k}} \sum_{\alpha=i+1}^{\alpha=n} \omega_{\alpha} e^{\rho \omega_{\alpha}(x-\xi)}\right\}\left\{p_{2} \rho^{n-2} Z_{n-2}+\right. \\
\left.\cdots+p_{n} Z_{0}\right\} d \xi .
\end{array}
$$


If $m(\rho)$ is the maximum value taken on by $\left|Z_{k}(x, \rho)\right|, 0 \leqq x \leqq 1, k=0, \cdots$, $n-1$, Lemma I enables us to conclude that

$$
\begin{aligned}
\left|Z_{k}\right| \leqq & \frac{1}{n|\rho|} C i \int_{0}^{x}\left\{\left|p_{2}\right|+\cdots+\frac{\left|p_{n}\right|}{|\rho|^{n-2}}\right\} d \xi \cdot m(\rho) \\
& +\frac{1}{n|\rho|} C(n-i) \int_{x}^{1}\left\{\left|p_{2}\right|+\cdots+\frac{\left|p_{n}\right|}{|\rho|^{n-2}}\right\} d \xi \cdot m(\rho) .
\end{aligned}
$$

For some values of $x$ and $k$ we can always write $\left|Z_{k}\right|=m(\rho)$, so that

$$
m(\rho) \leqq m(\rho) \frac{C}{|\rho|} \int_{0}^{1}\left\{\left|p_{2}\right|+\cdots+\frac{\left|p_{n}\right|}{|\rho|^{n-2}}\right\} d \xi \leqq m(\rho) \frac{K}{|\rho|} .
$$

This is impossible for large values of $|\rho|$ unless $m(\rho)=0$. Thus $U \equiv 0$. Under the second of the two alternatives above we should have $C_{1} \bar{u}_{1}+\cdots+C_{n} \bar{u}_{n}$ identically zero without having $C_{1}, \cdots, C_{n}$ all identically zero. By hypothesis this is impossible. The first alternative alone is possible. Consequently we have $u \equiv C_{1} \bar{u}_{1}+\cdots+C_{n} \bar{u}_{n}$ as we wished to show. We may remark that, since the coefficients $\bar{c}_{1}, \cdots, \bar{c}_{n}$ associated with $u$ were assumed to be constants, we can assert that $C_{1}, \cdots, C_{n}$ are analytic for all $\rho$ of sufficiently great absolute value so that $u$ is also analytic in $\rho$. This completes the theorem.

We shall need in some of our work a slightly more detailed form of the theorem actually stated by Birkhoff.

THEOREM III'. If $p_{2}, \cdots, p_{n}$ are real or complex functions of the real variable $x, 0 \leqq x \leqq 1$, continuous together with their derivatives of all orders, and if $\rho$ is a complex parameter, then the differential equation

$$
u^{(n)}+\star+p_{2} u^{(n-2)}+\cdots+p_{n-1} u^{(1)}+\left(p_{n}+\rho^{n}\right) u=0
$$

has on any region $T$ of the complex $\rho$-plane $n$ linearly independent solutions analytic in $\rho, u_{1}, \cdots, u_{n}$, expressible with all their derivatives in the form

$$
\begin{aligned}
u_{i}^{(k)}(x, \rho)=\left(\rho \omega_{i}\right)^{k} e^{\rho \omega_{i} x}\{1+ & \left.\sum_{l=1}^{l=m} \frac{A_{l k}(x)}{\left(\rho \omega_{i}\right)^{l}}+\frac{E_{i k}(x, \rho)}{\rho^{m+1}}\right\} \\
& (i=1, \cdots, n ; k, m=0,1,2, \cdots),
\end{aligned}
$$

wnere $A_{l k}(x)$ is continuous together with its derivatives of all orders and $E_{i k}(x, \rho)$ is bounded for all $\rho$ on $T,|\rho| \geqq R$, and all $x$ on $(0,1)$. The functions $A_{\boldsymbol{u}}(x)$ may be taken independent of the particular region $T$.

To demonstrate this theorem we first determine a function

$$
\bar{u}_{1}(x, \rho)=e^{\rho \omega_{1} x}\left\{1+\sum_{l=1}^{l=m} \frac{A_{l 0}(x)}{\left(\rho \omega_{1}\right)^{l}}\right\}
$$


with the property that when substituted in the differential expression which is the left-hand member in our differential equation it gives rise to a series in powers of $\rho$ beginning with a term in $\left(\rho \omega_{1}\right)^{n-m-2}$ at most. Since the substitution of $\rho \omega_{i} / \omega_{1}$ for $\rho$ in the differential expression does not change it, the functions $\bar{u}_{i}=\bar{u}_{1}\left(x, \rho \omega_{i} / \omega_{1}\right)$ have the same property. Taking $\bar{u}_{1}, \cdots, \bar{u}_{n}$ as approximants to $u_{1}, \cdots, u_{n}$ we then apply the reasoning of Birkhoff's paper to establish the theorem for $k=0, \cdots, n-1$. For $k=n$ we substitute the asymptotic forms thus obtained in the differential equation; for $k=n+1$ we differentiate the equation once with respect to $x$ and substitute the forms previously determined; continuing thus, we establish the theorem for all values of $k$ by induction. Since the same functions $\bar{u}$, numbered in accordance with the ordering of the constants $\omega$, can be employed for any other region $T$, the functions $A_{l k}(x)$ are independent of the particular region.

We may notice that, if we determine beforehand the integer $n$ and the greatest value of $k$ which we wish to employ, the functions $p_{2}, \cdots, p_{n}$ do not need to be taken as continuous with continuous derivatives of all orders in applications of this theorem. For the sake of simplifying a discussion sufficiently complicated in other ways we make this more restrictive assumption.

\section{The general PROBlem}

The general problem which we shall discuss arises from a linear homogeneous differential system of the $n$th order:

$$
\begin{aligned}
u^{(n)}+\star+p_{2} u^{(n-2)}+\cdots+\left(p_{n}+\lambda\right) u & =0, \quad 0 \leqq x \leqq 1, \\
W_{1}(u)=0, \quad \cdot \quad \cdot \quad W_{n}(u) & =0,
\end{aligned}
$$

where $W_{1}(u), \cdots, W_{n}(u)$ are linearly independent homogeneous linear forms in $u(0), \cdots, u^{(n-1)}(0), u(1), \cdots, u^{(n-1)}(1)$ with real or complex constant coefficients. There is no loss of generality in restricting attention to the unit interval. As we have already pointed out in $\S I$ there is associated with this system a Green's function except when the boundary conditions are identically satisfied by some solution of the differential equation; this Green's function will have the essential properties of the Green's function defined in the case of an equation with continuous coefficients. We now make the assumption that the Green's function $G(x, y ; \lambda)$ has infinitely many poles in the $\lambda$-plane, $\lambda_{1}, \lambda_{2}, \cdots$, which can be arranged so that $\left|\lambda_{1}\right| \leqq\left|\lambda_{2}\right|$ $\leqq \cdots, \lim _{\nu \rightarrow \infty}\left|\lambda_{\nu}\right|=\infty$. We let $R_{\nu}(x, y)$ be the residue at $\lambda=\lambda_{\nu}$; considered as a function of $x$, this residue satisfies the differential system for $\lambda=\lambda_{\nu}$. We next define a system of circles $C_{1}, C_{2}, \cdots$, with centers at $\lambda=0$ and radii $\Lambda_{1}, \Lambda_{2}, \cdots$ forming a monotone sequence with limit $+\infty$. We may suppose 
further that the region between two consecutive circles of the system contains at least one pole of $G$ and as few others as possible. No pole shall lie on any of the circles $C$. For any function $f(x)$ summable on $(0,1)$, and for that branch of the function $\left[1-\left(\lambda^{4} / \Lambda_{\nu}{ }^{4}\right)\right]^{k+l}$ reducing to 1 at the origin, we consider the behavior of the integrals

$$
\begin{gathered}
\frac{1}{2 \pi i} \frac{\partial^{k}}{\partial x^{k}} \int_{0}^{1} f(y) \int_{C_{\nu}}\left(1-\frac{\lambda^{4}}{\Lambda_{\nu}^{4}}\right)^{k+l} G(x, y ; \lambda) d \lambda d y, \quad l \geqq 0, \\
\frac{1}{2 \pi i} \int_{0}^{1} f(y) \int_{C_{\nu}} G(y, x ; \lambda) d \lambda d y,
\end{gathered}
$$

as $\nu \rightarrow \infty$. By the theory of residues these expressions are respectively

$$
\begin{aligned}
& \sum_{\alpha=1}^{\alpha=n_{y}}\left(1-\frac{\lambda_{\alpha}^{4}}{\Lambda_{\nu}^{4}}\right)^{k+l} \int_{0}^{1} f(y) \frac{\partial^{k}}{\partial x^{k}} R_{\alpha}(x, y) d y, \\
& \sum_{\alpha=1}^{\alpha=n_{y}} \int_{0}^{1} f(y) R_{\alpha}(y, x) d y,
\end{aligned}
$$

where $n_{\nu}$ is the number of poles of the Green's function in the circle $C_{\boldsymbol{v}}$.

To compare these expressions, suitably restricted in the case $k \neq 0$, with corresponding expressions in the theory of Fourier series we construct a differential system of order $n$ for which the integrals and series just described become Fourier series. We then study the difference of the corresponding expressions formed for the two differential systems.

We shall, in the present paper, restrict ourselves to those differential systems for which the boundary conditions $W_{1}, \cdots, W_{n}$, after being reduced to their normal form, are regular according to Birkhoff's definition.* The differential system from which we obtain the Fourier series will be of this type. At a later time we shall come back to the irregular cases, $n=2$.

Our fundamental tool for this investigation is a theorem due to Lebesgue. $\dagger$ It is sufficient for our purposes to quote it, with minor modifications.

THEOREM IV. Let there be given a function $\phi(x, y, \nu)$ defined for $x$ and $y$ on the interval $(a, b)$ and for real positive values of $\nu$ belonging to a set $N$ one of whose limit points is $+\infty$; and let $\phi$ be summable in $y$ for each pair of values $(x, \nu)$. Then a sufficient condition that, as $\nu$ becomes infinite in any manner in $N$,

$$
\lim _{\nu \rightarrow \infty} \int_{a}^{b} f(y) \phi(x, y, \nu) d y=0
$$

* For the terms normal and regular, see Birkhoff, these T r a n s a c t i o n s, vol. 9 (1908), pp. 382-383.

† Lebesgue, Annales de Toulouse, ser. 3, vol. 1 (1909), pp. 52-55. 
uniformly for all $x$ belonging to a set $E$ on $(a, b), f(x)$ being any summable function, is that

(1) $|\phi(x, y, \nu)| \leqq M$ for all $\nu$ in $N$ and for all $x$ in $E$, except for values of $y$ on a set $E^{\prime}$ of zero measure;

(2) $\lim _{\nu \rightarrow \infty} \int_{\alpha}^{\beta} \phi(x, y, \nu) d y=0$ uniformly for $x$ in $E, a \leqq \alpha<\beta \leqq b$.

Lebesgue also shows these conditions necessary, but we shall not use this fact.

\section{FourIER SERIES}

We find in the following theorem a generalisation of facts well known in the cases $n=1$ and $n=2$ :

Theorem V. The expansion problem associated with the differential system

$$
\begin{aligned}
& u^{(n)}+\lambda u=0, \\
& u(0)-u(1)=0, \\
& \cdot \cdot \cdot \cdot \cdot \cdot \cdot \\
& u^{(n-1)}(0)-u^{(n-1)}(1)=0,
\end{aligned}
$$

in which the boundary conditions are regular, gives rise to Fourier series.

The regularity of the boundary conditions is a matter of direct computation which we omit. For the case where $n$ is even this has been carried through elsewhere.*

We first determine the values of $\lambda$ for which the differential system has solutions not identically zero. When $\lambda=0$, it is clear that the only solution of the system is the solution $u=$ constant. When $\lambda \neq 0$ we make the substitution $\lambda=\rho^{n}$. Choosing $n$ linearly independent solutions of the differential equation, $u_{1}=e^{\rho \omega_{1} x}, \cdots, u_{n}=e^{\rho \omega_{n} x}$, we find all the other characteristic values of $\lambda=\rho^{n}$ from the roots of the equation

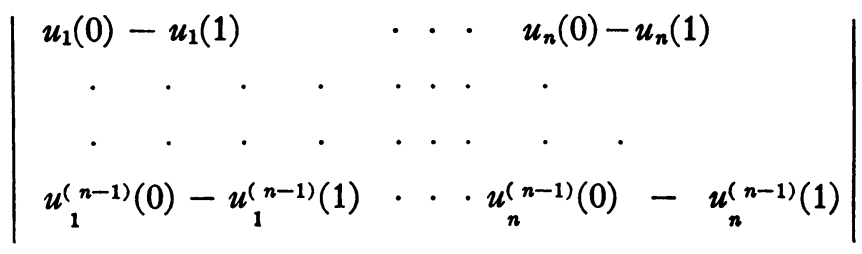

$$
\begin{aligned}
& \equiv \Omega_{n} \rho^{n(n-1) / 2}\left(1-e^{\rho \omega_{1}}\right) \cdot \cdots\left(1-e^{\rho \omega_{n}}\right)
\end{aligned}
$$

where $\Omega_{n}$ is a constant not zero. The roots of this equation other than $\rho=0$ are $\rho=2 \nu \pi i / \omega_{k}, k=1, \cdots, n, \nu= \pm 1, \pm 2, \cdots$ The corresponding values of $\lambda$ are $\lambda=-(2 \nu \pi i)^{n}, \nu= \pm 1, \pm 2, \cdots$.

* Tamarkin, Rendiconti del Circolo Matematico di Palermo, vol. 34 (1912), p. 359. 
Hence the Green's function $G(x, y ; \lambda)$ exists for this differential system.

We consider separately the two cases $n=2 \mu-1, n=2 \mu$.

Case I. $n=2 \mu-1$. Corresponding to $\lambda=\lambda_{\nu}=-(2 \nu \pi i)^{n}, G(x, y, \lambda)$ has a residue $R_{\nu}(x, y)$; and the differential system a solution $e^{2 \pi i x}, \nu=0, \pm 1$, ... We use these facts to obtain a development of $R_{\nu}(x, y)$, considered as a function of $y$, in Fourier series. By a fundamental property of Green's function we have

$$
\left(\lambda-\lambda_{\nu^{\prime}}\right) \int_{0}^{1} e^{2 \nu^{\prime} \pi i y} G(x, y ; \lambda) d y=e^{2 \nu^{\prime} \pi i x} .
$$

We divide this equation by $2 \pi i\left(\lambda-\lambda_{\nu^{\prime}}\right)$ and integrate both sides of the resulting equality with respect to $\lambda$ over a small circle surrounding the point $\lambda=\lambda_{\nu}$. We obtain

$$
\int_{0}^{1} e^{2 \nu^{\prime} x^{\prime} i \nu} R_{\nu}(x, y) d y=\left\{\begin{array}{ll}
e^{2 \nu x i x}, & \nu^{\prime}=\nu \\
0, & \nu^{\prime} \neq \nu
\end{array}\right\} .
$$

If we form the corresponding equations for $-\nu^{\prime}$, add them to those just written down and divide by two, we find

$$
\int_{0}^{1} \cos 2 \nu^{\prime} \pi y R_{\nu}(x, y) d y=\left\{\begin{array}{ll}
\frac{1}{2} e^{2 \nu \pi i x}, & \nu^{\prime}= \pm \nu \\
0 & , \nu^{\prime} \neq \pm \nu
\end{array}\right\} .
$$

In the same way

$$
\int_{0}^{1} \sin 2 \nu^{\prime} \pi y R_{\nu}(x, y) d y=\left\{\begin{array}{cc}
-\frac{i}{2} e^{2 \nu \pi i x}, & \nu^{\prime}=+\nu \\
+\frac{i}{2} e^{2 \nu x i x}, & \nu^{\prime}=-\nu \\
0 & , \nu^{\prime} \neq \pm \nu
\end{array}\right\} .
$$

Hence we see that $R_{\nu}(x, y)=e^{2 v i(x-y)}, \nu=0, \pm 1, \pm 2, \cdots$. If now we let $C_{0}$ be a circle with center at the origin in the $\lambda$-plane, including no other pole of $G(x, y ; \lambda), C_{1}$ a concentric circle including also $\lambda_{1}, \lambda_{-1}$, and so on, we have to consider the expressions

$$
\begin{gathered}
\frac{1}{2 \pi i} \frac{\partial^{k}}{\partial x^{k}} \int_{0}^{1} f(y) \int_{C_{\nu}}\left(1-\frac{\lambda^{4}}{\Lambda_{\nu}^{4}}\right)^{k+l} G(x, y ; \lambda) d \lambda d y, \\
\frac{1}{2 \pi i} \int_{0}^{1} f(y) \int_{C_{\nu}} G(y, x ; \lambda) d \lambda d y .
\end{gathered}
$$


On writing $\Lambda_{\nu}=\left(2 \pi \omega_{\nu}\right)^{n}$ and denoting by $\left[\omega_{\nu}\right]$ the greatest integer less than $\omega_{\nu}$, we see that the first integral reduces to

$$
\int_{0}^{1} f(y) d y+2 \sum_{\alpha=1}^{\alpha=\left[\omega_{\nu}\right]}\left(1-\frac{\alpha^{4 n}}{\omega_{\nu}^{4 n}}\right)^{k+l} \int_{0}^{1} f(y) \frac{\partial^{k}}{\partial x^{k}} \cos 2 \alpha \pi(x-y) d y,
$$

and the second to a similar series. We recognize the Fourier series for the interval $(0,1)$.

Case II. $n=2 \mu$. For $\lambda=\lambda_{\nu}=-(2 \nu \pi i)^{n}, \nu=0,1,2, \cdots$, the Green's function has residues $R_{\nu}(x, y)$; and the differential system has solutions $\cos 2 \nu \pi x, \sin 2 \nu \pi x$. From the identities

$$
\begin{aligned}
& \left(\lambda-\lambda_{\nu^{\prime}}\right) \int_{0}^{1} \cos 2 \nu^{\prime} \pi y G(x, y ; \lambda) d y=\cos 2 \nu^{\prime} \pi y, \\
& \left(\lambda-\lambda_{\nu^{\prime}}\right) \int_{0}^{1} \sin 2 \nu^{\prime} \pi y G(x, y ; \lambda) d y=\sin 2 \nu^{\prime} \pi x,
\end{aligned}
$$

we obtain, as in Case $\mathrm{I}$, the Fourier development of $R_{\nu}(x, y)$ considered as a function of $y$. It turns out that

$$
R_{0}(x, y)=1, \quad R_{\nu}(x, y)=2 \cos 2 \nu \pi(x-y), \quad \nu=1,2, \cdots .
$$

As in Case I, the expansion problems lead us directly to Fourier series for the interval $(0,1)$.

To make clear the nature of the expressions involving factors of the form $\left[1-\left(\alpha^{4 n} / \omega^{4 n}\right)\right]^{k+l}$ we give references to the literature from which the following theorem may be proved:

Theorem VI. Let $u_{0}(x)+u_{1}(x)+u_{2}(x)+\cdots$ be an infinite series whose terms are real functions of the real variable $x, a \leqq x \leqq b$. Then the expressions

$$
S_{\Omega}^{\delta, \Delta}(x) \equiv \sum_{\alpha=0}^{[\omega]}\left(1-\frac{\alpha^{\Delta}}{\Omega}\right)^{\delta} u_{\alpha}(x)
$$

where $\Omega=\omega^{\Delta}, \Delta>0, \delta \geqq 0$, are the Riesz typical means of the first kind of order $\delta$ and type $\alpha^{\Delta}$ for the infinite series in question. If $\lim _{\Omega \rightarrow \infty} S_{\Omega}^{\delta, \Delta}$ exists and is equal to $U(x)$, the infinite series is said to be summable $\left(\alpha^{\Delta}, \delta\right)$ at the point $x$ to the value $U(x)$. Summability $\left(\alpha^{\Delta}, \delta\right)$ implies summability $\left(\alpha^{\Delta^{\prime}}, \delta\right)$ to the same value; if the summability $\left(\alpha^{\Delta}, \delta\right)$ is uniform for a certain range of values of $x$, then the summability $\left(\alpha^{\Delta^{\prime}}, \delta\right)$ is also uniform for that range. Likewise, summability $\left(\alpha^{\Delta}, \delta\right)$ implies summability $\left(\alpha^{\Delta}, \delta^{\prime}\right)$ to the same value if $\delta^{\prime} \geqq \delta$, with similar remarks on uniform summability. The means $S_{\Omega}^{\delta, 1}(x)$ are equivalent to the Cesàro means for integral $\delta$ and their generalisations for arbitrary $\delta$; that is to say, summability $(\alpha, \delta)$ implies summability $C(\delta)$ to the same value, and conversely, with remarks on uniform summability like those previously made. 
Riesz's typical means are discussed in some detail in a joint work of Hardy and Riesz,* where further references are given. $\dagger$ The series there considered are series of constants; but the extension of the methods employed to questions of uniform summability when series of functions are involved requires merely appropriate modification of Lemmas $4,5,7,8$. Theorem 16 gives the relation between summability $\left(\alpha^{\Delta}, \delta\right)$ and summability $\left(\alpha^{\Delta}, \delta^{\prime}\right)$ when $\delta^{\prime} \geqq \delta$. The relation between summability $\left(\alpha^{\Delta}, \delta\right)$ and summability $\left(\alpha^{\Delta^{\prime}}, \delta\right)$ is a special case of the theorem stated in the second foot-note on page 33; a proof of this fact could be modeled on that of Theorem 17. The comparison of summability $(\alpha, \delta)$ with summability $C(\delta)$ follows a paper of Riesz. $\ddagger$ We add the

Corollary I. Summability $\left(\alpha^{\Delta}, \delta\right)$ and summability $C(\delta)$ are completely equivalent with respect to summability at a point and uniform summability over a set of points; each implies the other.

The importance of this corollary is due to the fact that the known results concerning the Cesàro summability of Fourier series and its term-by-term derived series can be carried over to summability by the Riesz means, which are better suited to the form of Fourier series which we shall discuss under Theorem V. We shall then be able to carry these results over to the general Birkhoff series by the intervention of this corollary and Theorem V.

\section{BIRKHOFF SERIES FOR $n=2 \mu-1$}

Distinct differences between the case when $n$ is odd and the case when $n$ is even make it necessary to consider each case separately. The methods employed in the two cases are virtually the same; we therefore give a complete study of the case $n=2 \mu-1$ and then describe the necessary changes in mode of attack and in statement of theorems in the case $n=2 \mu$.

By our hypotheses, the differential system considered is

$$
\begin{gathered}
u^{(n)}+\star+p_{2} u^{(n-2)}+\cdots+\left(p_{n}+\lambda\right) u=0, \\
W_{1}(u)=0, \cdots, W_{n}(u)=0, n=2 \mu-1,
\end{gathered}
$$

* Hardy and Marcel Riesz, The General Theory of Dirichlet's Series (Cambridge Tracts, No. 18, 1915), Chapters IV and V.

† See especially Marcel Riesz, Sur les séries de Dirichlet et les séries entières, Comptes Rendus, vol. 149 (1909), pp. 909-912.

$\ddagger$ Marcel Riesz, Une méthode de sommation équivalente à la méthode des moyens arithmétiques, Comptes Rendus, vol. 152 (1911), pp. 1651-1654. 
where

$$
\begin{aligned}
& W_{i}(u) \equiv \alpha_{i} u^{\left(k_{i}\right)}(0)+\beta_{i} u^{\left(k_{i}\right)}(1)+\cdots, \\
& n-1 \geqq k_{1} \geqq k_{2} \geqq \cdots \geqq k_{n}, \quad k_{i} \neq k_{i+2},
\end{aligned}
$$

and $\alpha_{1}, \cdots, \alpha_{n}, \beta_{1}, \cdots, \beta_{n}, k_{1}, \cdots, k_{n}$ are subjected to certain conditions in accordance with the definition of regularity.* On making the substitution $\lambda=\rho^{n}$ we are led to consider instead of the entire $\lambda$-plane a corresponding sector of the $\rho$-plane, which we shall take as composed of two adjacent sectors $S: R\left(\rho \omega_{1}\right) \leqq R\left(\rho \omega_{2}\right) \leqq \cdots \leqq R\left(\rho \omega_{n}\right)$, arising, of course, from distinct orderings of the constants $\omega$; this region we call $\Sigma$. The sector $S$ is a region $T$ of $\S I$ for $c=0$. We recall now the facts concerning the asymptotic distribution of the values of $\rho$ giving rise to the characteristic values of $\lambda$. On an arbitrary sector $S$ these values of $\rho$ lie asymptotically near points equally spaced at distance $2 \pi$ along a line parallel to the bisecting ray of $S$; there is one and only one value of $\rho$ near each of these points when $|\rho|$ is large; and the corresponding values of $\lambda$ are simple characteristic values of the differential system. $\dagger$

We wish to compare any two given differential systems of order $n=2 \mu-1$. From the $\rho$-plane we remove all the points which give characteristic values of one system or the other of the two which we are comparing; we do this by removing the interiors of circles $\sigma$ of arbitrarily small radius $\epsilon$, one described about each such point as center. The portions of $S, \Sigma$ which remain we denote by $S^{\prime}, \Sigma^{\prime}$. We then denote by $\Gamma$ any circular arc on $\Sigma^{\prime}$ with center at the origin terminated by the rectilinear portions of the boundary of $\Sigma^{\prime}$. The image of $\Gamma$ in the $\lambda$-plane is a circle $C$; the totality of such circles $C$ forms an infinite set of concentric annular regions, none of which contains a characteristic value. From the behavior of the characteristic values for large $|\lambda|$ we see that the large circles of the set $C_{1}, C_{2}, C_{3}, \ldots$ as described in $\S I I$ can be selected as circles $C$; this is true simultaneously for the two differential systems we are considering.

The region $S$ is such that on it $\ddagger$

$$
\begin{aligned}
& R\left(\rho \omega_{1}\right) \leqq R\left(\rho \omega_{2}\right) \leqq \cdots \leqq R\left(\rho \omega_{\mu-1}\right) \leqq-\beta<0 ; \\
& R\left(\rho \omega_{\mu}\right)=0 \text { on the bisecting ray of } S ; \\
& R\left(\rho \omega_{n}\right) \geqq \cdots \geqq R\left(\rho \omega_{\mu+1}\right) \geqq \beta>0 .
\end{aligned}
$$

* Birkhoff, these Transactions, vol. 9 (1908), p. 383.

$\dagger$ Birkhoff, these Transactions, vol. 9 (1908), pp. 383-386.

‡ Birkhoff, these Transactions, vol. 9 (1908), p. 384. 
We therefore denote by $S_{1}$ that part of $S$ for which $R\left(\rho \omega_{\mu}\right) \leqq 0$; the part for which $R\left(\rho \omega_{\mu}\right) \geqq 0$ by $S_{2}$. The notations $S_{1}{ }^{\prime}, S_{2}{ }^{\prime}$ can be introduced according to the principle previously employed. The portions of $\Gamma$ on $S^{\prime}, S_{1}{ }^{\prime}, S_{2}{ }^{\prime}$, we shall call $\gamma, \gamma_{1}, \gamma_{2}$ respectively. The common radius of these arcs we denote by $R$.

We now prove several preparatory lemmas.

LEMMA III. If $m(x, y, \rho)$ is a function of $x, y$, and $\rho$ bounded for $a \leqq x \leqq b$, $a \leqq y \leqq b$, and for all $\rho$ on $S^{\prime}$ such that $\theta^{\prime} \leqq \arg \rho \leqq \theta^{\prime \prime} ;$ and if $\alpha$ is any arc of $\gamma$ between $\theta^{\prime}$ and $\theta^{\prime \prime}$, then the integral $\int_{\alpha} m(x, y, \rho) d \rho / \rho$ is a bounded function of $x, a \leqq x \leqq b, y, a \leqq y \leqq b$, and $R$.

We have

$$
\left|\int_{\alpha} m \frac{d \rho}{\rho}\right| \leqq M \int_{\alpha} d \theta \leqq M\left(\theta^{\prime \prime}-\theta^{\prime}\right) .
$$

LEMMA IV. If $m(x, \rho)$ is a function of $x$ and $\rho$ bounded for $a \leqq x \leqq b$, $0<a<b<1$, and for all $\rho$ on $S^{\prime}$ such that $\theta^{\prime} \leqq \arg \rho \leqq \theta^{\prime \prime}$, then

$$
\begin{array}{ll}
\lim _{R \rightarrow \infty} \int_{\alpha} \rho^{k} e^{\rho \omega_{i} x} m(x, \rho) d \rho=0 & (i=1, \cdots, \mu-1), \\
\lim _{R \rightarrow \infty} \int_{\alpha} \rho^{k} e^{\rho \omega_{i}(x-1)} m(x, \rho) d \rho=0 & (i=\mu+1, \cdots, n),
\end{array}
$$

uniformly, $0<a \leqq x \leqq b<1$, for any positive $k$.

We let $\delta$ be the lesser of $a, 1-b$. Then

$$
\left|\int_{\alpha} \rho^{k} e^{\rho \omega_{i} x} m d \rho\right| \leqq M R^{k+1} e^{-R \beta \delta} \int_{\alpha} d \theta \leqq M R^{k+1} e^{-R \beta \delta}\left(\theta^{\prime \prime}-\theta^{\prime}\right) \rightarrow 0
$$

when $i=1, \cdots, \mu-1$; and

$$
\left|\int_{\alpha} \rho^{k} e^{\omega_{i}(x-1)} m d \rho\right| \leqq M\left(\theta^{\prime \prime}-\theta^{\prime}\right) R^{k+1} e^{-R \beta \delta} \rightarrow 0
$$

when $i=\mu+1, \cdots, n$.

LEMMA V. If $m(x, \rho)$ is a function of $x$ and $\rho$ bounded for $0<a \leqq x \leqq b<1$, and for all $\rho$ on $S_{1}^{\prime}$, then

$$
\int_{\alpha} \rho^{k}\left(1-\frac{\rho^{4 n}}{R^{4 n}}\right)^{k+l} e^{\rho \omega \mu} x^{\rho} m(x, \rho) d \rho
$$

for any $k \geqq 0,(1)$ is uniformly bounded for all $\alpha$ on $\gamma_{1}$ and all $x$ on $(a, b)$ if $l \geqq 0$, and (2) approaches zero uniformly as $R \rightarrow \infty$ if $l>0$. If $m(x, \rho)$ is a function 
of $x$ and $\rho$ bounded for $x$ on $(a, b)$ and for all $\rho$ on $S_{2}^{\prime}$ similar statements are true of the integral

$$
\int_{\alpha} \rho^{k}\left(1-\frac{\rho^{4 n}}{R^{4 n}}\right)^{k+l} e^{\rho \omega_{\mu}(x-1)} m(x, \rho) d \rho .
$$

In the case of the first integral we introduce an angle $\phi$ measured from the bisecting ray of $S$ as initial direction, with the positive sense in $S_{1}$. We then see that we can write

$$
\begin{aligned}
& \rho \omega_{\mu}=i R e^{i \phi}, \\
& 0 \leqq \phi / 2 \leqq \sin \phi, \quad 0 \leqq \phi \leqq \frac{\pi}{2 n}, \\
& 0 \leqq\left|1-e^{4 n i \phi}\right|^{k+l} \leqq N \phi^{k+l}, \quad 0 \leqq \phi \leqq \frac{\pi}{2 n} .
\end{aligned}
$$

We find

$$
\begin{aligned}
& \left|\int_{\alpha} \rho^{k}\left(1-\frac{\rho^{4 n}}{R^{4 n}}\right)^{k+l} e^{\rho \omega \mu} x m d \rho\right| \\
& =\left|\int_{\phi^{\prime}}^{\phi^{\prime \prime}} R^{k+1}\left(1-e^{4 n i \phi}\right)^{k+l} e^{(k+1) i \phi} e^{-R x \sin \phi} e^{i R x \cos \phi} m d \phi\right| \\
& \leqq M \int_{0}^{\pi / 2 n} R^{k+1}\left|1-e^{4 n i \phi}\right|^{k+l} e^{-R \delta \phi / 2} d \phi \leqq M N \int_{0}^{\pi / 2 n} R^{k+1} \phi^{k+l} e^{-R \delta \phi / 2} d \phi \\
& =\frac{M N}{R^{l}} \int_{0}^{\pi R / 2 n} \xi^{k+l} e^{-\delta \xi / 2} d \xi \leqq \frac{M N}{R^{l}} \int_{0}^{\infty} \xi^{k+l} e^{-\delta \xi / 2} d \xi=\frac{K}{R^{l}} .
\end{aligned}
$$

This establishes the first result. The second integral can be treated in precisely similar fashion.

LEMma VI. If $m(x, y, \rho)$ is bounded $0 \leqq x \leqq 1,0 \leqq y \leqq 1$, for all $\rho$ on $S$, then

$$
\int_{\alpha}^{x} \int_{\gamma_{1}} e^{\rho \omega_{i}(x-y)} \frac{m(x, y, \rho)}{\rho} d \rho d y
$$

approaches zero uniformly, $i=1, \cdots, \mu, 0 \leqq \alpha \leqq x \leqq 1$, and $i=\mu+1, \cdots, n$, $0 \leqq x \leqq \alpha \leqq 1 ;$ and

$$
\int_{\alpha}^{x} \int_{\gamma_{2}} e^{\rho \omega_{i}(x-y)} \frac{m(x, y, \rho)}{\rho} d \rho d y
$$

approaches zero uniformly, $i=1, \cdots, \mu-1,0 \leqq \alpha \leqq x \leqq 1$, and $i=\mu, \cdots, n$, $0 \leqq x \leqq \alpha \leqq 1$. 
We have when $i=1, \cdots, \mu-1,0 \leqq \alpha \leqq x \leqq 1$,

$$
\begin{gathered}
\left|\int_{\alpha}^{x} \int_{\gamma_{1}} e^{\rho \omega_{i}(x-y)} \frac{m}{\rho} d \rho d y\right| \leqq \int_{\alpha}^{x} \int_{0}^{\pi / 2 n} e^{-R \beta(x-y)} M d \phi d y \\
=\frac{\pi M}{2 n} \frac{1-e^{-R \beta(x-\alpha)}}{R \beta} \leqq \frac{\pi M}{2 R \beta n} \rightarrow 0 .
\end{gathered}
$$

Similar reasoning applies when $i=\mu+1, \cdots, n, 0 \leqq x \leqq \alpha \leqq 1$. The integrals over $\gamma_{2}, i \neq \mu$, are discussed in the same way.

When $i=\mu$ the treatment is slightly different. For $0 \leqq \alpha \leqq x \leqq 1$

$$
\begin{aligned}
& \left|\int_{\alpha}^{x} \int_{\gamma_{1}} e^{\rho \omega_{\mu}(x-y)} \frac{m}{\rho} d \rho d y\right| \leqq \int_{\alpha}^{x} \int_{0}^{\pi / 2 n} e^{-R \varphi(x-y) / 2} M d \phi d y \\
& \quad=M \int_{\alpha}^{x} \frac{1-e^{-\pi R(x-y) / 4 n}}{R(x-y)} d y=\frac{M}{R} \int_{\alpha}^{R(x-\alpha)} \frac{1-e^{-\pi \xi / 4 n}}{\xi} d \xi \\
& \quad \leqq \frac{M}{R} \int_{0}^{R} \frac{1-e^{-\pi \xi / 4 n}}{\xi} d \xi=\frac{M}{R} O(\log R) \rightarrow 0 .
\end{aligned}
$$

By analogous means we obtain the corresponding result for the integral over $\boldsymbol{\gamma}_{2}$.

We can now demonstrate

THEOREM VII. If $G$ and $\bar{G}$ are the Green's functions for any two regular differential systems of order $n=2 \mu-1$, then for any region $S^{\prime}$ the integral

$$
\int_{\gamma} n \rho^{n-1}\left(1-\frac{\rho^{4 n}}{R^{4 n}}\right)^{l}\left\{G\left(x, y ; \rho^{n}\right)-\bar{G}\left(x, y ; \rho^{n}\right)\right\} d \rho
$$

is bounded for all $\gamma$ on $S^{\prime}, 0 \leqq y \leqq 1,0<a \leqq x \leqq b<1$, for $l \geqq 0$.

We first recall the explicit form of the Green's function, which we know to exist in the present case. We have

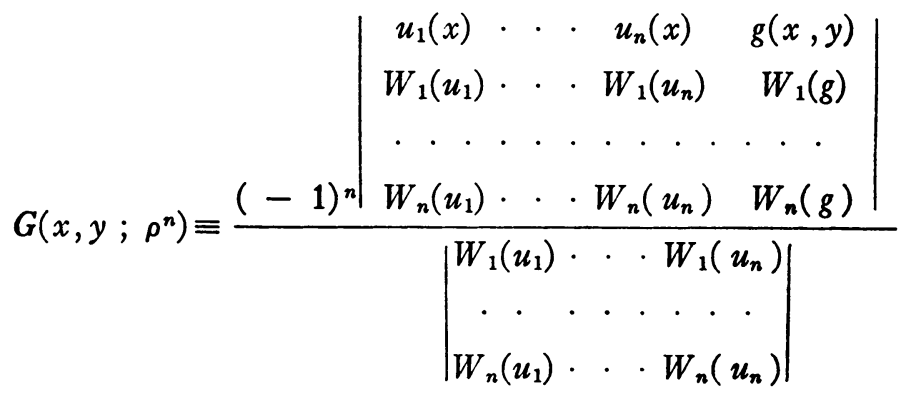


where $u_{1}, \cdots, u_{n}$ are $n$ linearly independent solutions of the differential equation and

$$
g(x, y) \equiv\left\{\frac{1}{2} \sum_{i=1}^{i=n} u_{i}(x) v_{i}(y) ;-\frac{1}{2} \sum_{i=1}^{i=n} u_{i}(x) v_{i}(y)\right\}
$$

the notation $\{A ; B\}$ indicating that $A$ is to be taken if $x \geqq y, B$ if $x \leqq y$, and the functions $v_{1}, \cdots, v_{n}$ being defined by the identity in $\tau_{1}, \cdots, \tau_{n}$

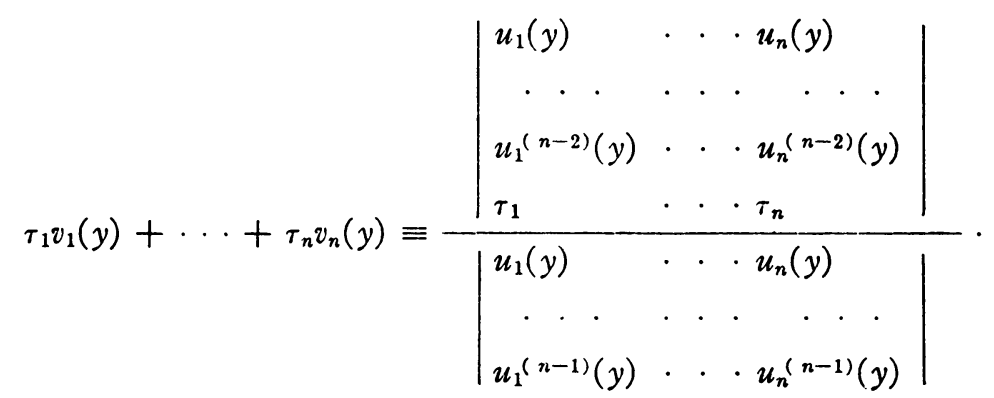

We shall take $u_{1}, \cdots, u_{n}$ as the functions defined in Theorem III for the sector $S$. It is then possible to determine the asymptotic form of the Green's function on $S_{1}{ }^{\prime}$ and $S_{2}{ }^{\prime}$ in sufficient detail that the present theorem is apparent from the lemmas just established.

Case I. $\rho$ on $S_{1}{ }^{\prime}$. We multiply the first $\mu$ columns in the numerator of $G$ by $\frac{1}{2} v_{1}(y), \cdots, \frac{1}{2} v_{\mu}(y)$, respectively, the next $\mu-1$ columns by $-\frac{1}{2} v_{\mu+1}(y)$, $\cdots,-\frac{1}{2} v_{n}(y)$ and add to the lasi. We then take the first term in the new last column outside the determinant. The result of substituting the asymp totic forms of $u_{1}, \cdots, u_{n}, v_{1}, \cdots, v_{n}$ in the expression thus obtained is*

$$
n \rho^{n-1} G=\left\{-\sum_{i=1}^{i=\mu} e^{\rho \omega_{i}(x-y)}\left[\omega_{i}\right] ;+\sum_{i=\mu+1}^{i=n} e^{\rho \omega_{i}(x-y)}\left[\omega_{i}\right]\right\}+\frac{\Delta_{1}}{\left[\theta_{0}\right]+e^{\rho \omega_{i}}\left[\theta_{1}\right]}
$$

where $\Delta_{1}$ is a determinant of order $n+1$, in which the element $a_{h j}, h=0$, $\cdots, n, j=1, \cdots, n+1$, may be described as follows:

$$
\begin{array}{r}
a_{0 j}=e^{\rho \mu \nu j x}[1], j=1, \cdots, \mu ; a_{0, j}=e^{\rho \omega j(x-1)}[1], j=\mu+1, \cdots, n ; \\
a_{0, n+1}=0 ;
\end{array}
$$

and for $h=1, \cdots, n$

$$
\begin{aligned}
a_{h i}=\left[\alpha_{h} \omega_{j}{ }^{k}\right], j=1, \cdots, \mu-1 ; a_{h \mu} & =\left[\alpha_{h} \omega_{\mu}{ }^{k}\right] \\
a_{h i}=\left[\beta_{h} \omega_{j}{ }^{k}\right], j=\mu+1, \cdots, n ; a_{h, n+1} & =\sum_{i=1}^{i=\mu \mu}\left[\beta_{h} \omega_{\mu}{ }^{k \omega_{h}}\right] ; \\
& -\sum_{i=\mu+1}^{i=n} e^{-\rho \omega_{i} \nu}\left[\alpha_{h} \omega_{i}{ }^{k}{ }^{h+1}\right]
\end{aligned}
$$

- Birkhoff, these Transactions, vol. 9 (1908) pp. 389-395. 
The notation [ ] is used to indicate the leading term of the asymptotic form in question. The exponential terms in the determinant $\Delta_{1}$ are bounded for all $x$ and $y$ on $(0,1)$ and for all $\rho$ on $S_{1}$; and $1 /\left\{\left[\theta_{0}\right]+e^{\rho \omega_{\mu}}\left[\theta_{1}\right]\right\}$, in which $\theta_{0} \theta_{1} \neq 0$ by the regularity of the boundary conditions, is bounded for all $\rho$ on $S_{1}{ }^{\prime}{ }^{*}$ Hence we are able to write

$$
\begin{aligned}
\left(1-\frac{\rho^{4 n}}{R^{4 n}}\right)^{l}\left(n \rho^{n-1} G-\left\{-\sum_{i=1}^{i=\mu} e^{\rho \omega_{i}(x-y)} \omega_{i} ;+\sum_{i=\mu+1}^{i=n} e^{\rho \omega_{i}(x-y)} \omega_{i}\right\}\right) \\
=\left\{-\sum_{i=1}^{i=\mu} e^{\rho \omega_{i}(x-y)} \frac{m_{i}(x, y, \rho)}{\rho} ;+\sum_{i=\mu+1}^{i=n} e^{\rho \omega_{i}(x-y)} \frac{m_{i}(x, y, \rho)}{\rho}\right\} \\
+\sum_{i=1}^{i=\mu} e^{\rho \omega_{i} x} M_{i}(x, y, \rho)+\sum_{i=\mu+1}^{i=n} e^{\rho \omega_{i}(x-1)} M_{i}(x, y, \rho)
\end{aligned}
$$

where $m$ and $M$ denote functions bounded for all $x$ and $y$ on $(0,1)$ and for all $\rho$ on $S_{1}{ }^{\prime}$. Thus, by Lemmas III, IV, V we see that

$$
\int_{\gamma_{1}}\left(1-\frac{\rho^{4 n}}{R^{4 n}}\right)^{l}\left(n \rho^{n-1} G-\left\{-\sum_{i=1}^{i=\mu} e^{\rho \omega_{i}(x-y)} \omega_{i} ;+\sum_{i=\mu+1}^{i=n} e^{\rho \omega_{i}(x-y)} \omega_{i}\right\} d \rho\right.
$$

is bounded $0 \leqq y \leqq 1,0<a \leqq x \leqq b<1$, for all $\gamma_{1}$ on $S_{1}{ }^{\prime}$. The application of Lemma III, it should be observed, is made to the bracket term on the right, which is clearly of the form $m / \rho$ for all $x$ and $y$ on $(0,1)$ and all $\rho$ on $S_{1}$.

Of course, in the result just obtained we may replace $G$ by $\bar{G}$; a combinationof the facts thus established shows that the integral

$$
\int_{\gamma_{1}} n \rho^{n-1}\left(1-\frac{\rho^{4 n}}{R^{4 n}}\right)^{l}(G-\bar{G}) d \rho
$$

is bounded $0 \leqq y \leqq 1,0<a \leqq x \leqq b<1$, for all $\gamma_{1}$ on $S_{1}{ }^{\prime}, l \geqq 0$.

Case II. $\rho$ on $S_{2}{ }^{\prime}$. We now multiply the first $\mu-1$ columns in the numerator determinant of $G$ by $\frac{1}{2} v_{1}(y), \cdots, \frac{1}{2} v_{\mu-1}(y)$ respectively, the next $\mu$ columns by $-\frac{1}{2} v_{\mu}(y), \cdots,-\frac{1}{2} v_{n}(y)$, and add to the last. We then continue as in Case I, finding

$$
n \rho^{n-1} G=\left\{-\sum_{i=1}^{i=\mu-1} e^{\rho \omega_{i}(x-\nu)}\left[\omega_{i}\right] ;+\sum_{i=\mu}^{i=n} e^{\rho \omega_{i}(x-y)}\left[\omega_{i}\right]\right\}+\frac{\Delta_{2}}{\left[\theta_{0}\right] e^{-\rho \omega_{\mu}}+\left[\theta_{1}\right]}
$$

where $\Delta_{2}$ differs from $\Delta_{1}$ in Case I in having the definition of $a_{h \mu}$ replaced by

$$
a_{0, \mu}=e^{\rho \omega_{\mu}(x-1)}[1] ; a_{h \mu}=e^{-\rho \omega_{\mu}}\left[\alpha_{h} \omega_{\mu}{ }^{k} h\right]+\left[\beta_{h} \omega_{\mu}{ }^{k}\right], h=1, \cdots, n,
$$

* Birkhoff, Rendiconti del Circolo Matematico di Palermo, vol. 36 (1913), pp. 120-121. 
and in having the sums in the definition of $a_{h, n+1}, h>0$, extended from $i=1$ to $i=\mu-1$ and from $i=\mu$ to $i=n$ respectively. The exponential terms in the determinant $\Delta_{2}$ are bounded for all $x$ and $y$ on $(0,1)$ and for all $\rho$ on $S_{2}{ }^{\prime}$; and $1 /\left\{\left[\theta_{0}\right] e^{-\rho \omega_{\mu}}+\left[\theta_{1}\right]\right\}$ is bounded for all $\rho$ on $S_{1}{ }^{\prime}$. The reasoning now proceeds as in Case $\mathrm{I}$. Thus we see that the integral

$$
\int_{\gamma_{2}} n \rho^{n-1}\left(1-\frac{\rho^{4 n}}{R^{4 n}}\right)^{l}(G-\bar{G}) d \rho
$$

is bounded $0 \leqq y \leqq 1,0<a \leqq x \leqq b<1$, for all $\gamma_{2}$ on $S_{2}{ }^{\prime}, l \geqq 0$.

By combining the facts in Cases I and II we obtain the theorem as stated for the integral over $\gamma$ on $S^{\prime}$. Since $S^{\prime}$ is arbitrary the result holds also for the arc $\Gamma$ on $\Sigma^{\prime}$ and can be interpreted in terms of the circles $C$ in the $\lambda$-plane.

We next demonstrate

TheOREM VIII. Under the hypotheses of Theorem VII, the integral

$$
\int_{\alpha}^{\beta} \int_{\gamma} n \rho^{n-1}\left(1-\frac{\rho^{4 n}}{R^{4 n}}\right)^{l}\left(G\left(x, y ; \rho^{n}\right)-\bar{G}\left(x, y ; \rho^{n}\right)\right) d \rho d y,
$$

where $l \geqq 0$ and $0 \leqq \alpha<\beta \leqq 1$, has the limit zero as $R \rightarrow \infty$, uniformly $0<a \leqq x$ $\leqq b<1$.

We split the integral over $(\alpha, \beta)$ into the sum of the integrals over $(\alpha, x)$ and $(x, \beta)$; since the last two integrals are of the same form we consider but one of them.

Case I. $\rho$ on $\gamma_{1}$. We employ the asymptotic form of $n \rho^{n-1} G$ obtained in the preceding theorem. In the determinant $\Delta_{1}$ the variable $y$ occurs only in the last column so that the integration with respect to $y$ can be performed directly in the determinant. We see at once that

$$
\begin{aligned}
\int_{x}^{\alpha} e^{\rho \omega_{i} y}\left(1+\frac{m}{\rho}\right) d y & =\frac{e^{\rho \omega_{i}(1-x)}-e^{\rho \omega_{i}(1-\alpha)}}{\rho \omega_{i}} \\
& +\int_{x}^{\alpha} e^{\rho \omega_{i}(1-y)} \frac{m}{\rho} d y=\frac{m(x, \alpha, \rho)}{R}
\end{aligned}
$$

for all $x$ and $\alpha$ on $(0,1)$ and for $\rho$ on $\gamma_{1}, i=1, \cdots, \mu$; and that

$$
\int_{x}^{\alpha} e^{-\rho \omega_{i} y}\left(1+\frac{m}{\rho}\right) d y=\frac{m(x, \alpha, \rho)}{R}
$$

for all $x$ and $\alpha$ on $(0,1)$ and for $\rho$ on $\gamma_{1}, i=\mu+1, \cdots, n$. Hence we see that 


$$
\begin{gathered}
\int_{x}^{\alpha} \int_{\gamma_{1}}\left(1-\frac{\rho^{4 n}}{R^{4 n}}\right)^{l}\left(n \rho^{n-1} G-\left\{-\sum_{i=1}^{i=\mu} e^{\rho \omega_{i}(x-y)} \omega_{i} ;+\sum_{i=\mu+1}^{i=n} e^{\rho \omega_{i}(x-y)} \omega_{i}\right\}\right) d \rho d y \\
=\int_{x}^{\alpha} \int_{\gamma_{1}}\left\{-\sum_{i=1}^{i=\mu} e^{\rho \omega_{i}(x-y)} \frac{m_{i}(x, y, \rho)}{\rho} ;+\sum_{i=\mu+1}^{i=n} e^{\rho \omega_{i}(x-y)} \frac{m_{i}(x, y, \rho)}{\rho}\right\} d \rho d y \\
\quad+\frac{1}{R} \int_{\gamma_{1}}\left(\sum_{i=1}^{i=\mu} e^{\rho \omega_{i} x} m_{n+i}(x, \alpha, \rho)+\sum_{i=\mu+1}^{i=n} e^{\rho \omega_{i}(x-1)} m_{n+i}(x, \alpha, \rho)\right) d \rho .
\end{gathered}
$$

The two expressions on the right of this equation approach zero uniformly as $R \rightarrow \infty, 0<a \leqq x \leqq b<1$, by Lemmas IV, V, VI.

We can replace $G$ by $\bar{G}$ in this result; and we may write $\beta$ instead of $\alpha$. Thus it follows that

$$
\lim _{R \rightarrow \infty} \int_{\alpha}^{\beta} \int_{\gamma_{1}} n \rho^{n-1}\left(1-\frac{\rho^{4 n}}{R^{4 n}}\right)^{l}\left(G\left(x, y ; \rho^{n}\right)-\bar{G}\left(x, y ; \rho^{n}\right)\right) d \rho d y=0
$$

uniformly $0<a \leqq x \leqq b<1$.

Case II. $\rho$ on $\gamma_{2}$. We must use here the asymptotic form of $n \rho^{n-1} G$ employed in Case II of the preceding theorem. We separate the integral over $(\alpha, \beta)$ into two parts as in Case I, and then perform the integration with respect to $y$ in the last column of the determinant $\Delta_{2}$. On expanding the determinant we have

$$
\begin{gathered}
\int_{x}^{\alpha} \int_{\gamma_{2}}\left(1-\frac{\rho^{4 n}}{R^{4 n}}\right)^{l}\left(n \rho^{n-1} G-\left\{-\sum_{i=1}^{i=\mu-1} e^{\rho \omega_{i}(x-y)} \omega_{i} ;+\sum_{i=\mu}^{i=n} e^{\rho \omega_{i}(x-y)} \omega_{i}\right\}\right) d \rho d y \\
=\int_{x}^{\alpha} \int_{\gamma_{2}}\left\{-\sum_{i=1}^{i=\mu-1} e^{\rho \omega_{i}(x-y)} \frac{m_{i}(x, y, \rho)}{\rho} ;+\sum_{i=\mu}^{i=n} e^{\rho \omega_{i}(x-y)} \frac{m_{i}(x, y, \rho)}{\rho}\right\} d \rho d y \\
+\frac{1}{R} \int_{\gamma_{2}}\left(\sum_{i=1}^{i=\mu-1} e^{\rho \omega_{i} x} m_{n+i}(x, \alpha, \rho)+\sum_{i=\mu}^{i=n} e^{\rho \omega_{i}(x-1)} m_{n+i}(x, \alpha, \rho)\right) d \rho .
\end{gathered}
$$

By Lemmas IV, V, VI, the expressions on the right approach zero uniformly as $R \rightarrow \infty, 0<a \leqq x \leqq b<1$. Thus, as in Case $\mathrm{I}$,

$$
\lim _{R \rightarrow \infty} \int_{\alpha}^{\beta} \int_{\gamma_{2}} n \rho^{n-1}\left(1-\frac{\rho^{4 n}}{R^{4 n}}\right)^{l}(G-\bar{G}) d \rho d y=0
$$

uniformly, $0<a \leqq x \leqq b<1$.

From Cases I and II we conclude the truth of the present theorem. By the arbitrariness of the sector $S^{\prime}$ the theorem remains true if the integration with respect to $\rho$ takes place over $\Gamma$. 
From the two theorems just established there follows

THEOREM IX. If $f(x)$ is any summable function defined on $(0,1)$ and if $l \geqq 0$, then

$$
\begin{aligned}
& \lim _{R \rightarrow \infty} \int_{0}^{1} f(y) \int_{\gamma} n \rho^{n-1}\left(1-\frac{\rho^{4 n}}{R^{4 n}}\right)^{l}\left(G\left(x, y ; \rho^{n}\right)-\bar{G}\left(x, y ; \rho^{n}\right)\right) d \rho d y=0, \\
& \lim _{\Lambda \rightarrow \infty} \int_{0}^{1} f(y) \int_{C}\left(1-\frac{\lambda^{4}}{\Lambda^{4}}\right)^{l}(G(x, y ; \lambda)-\bar{G}(x, y ; \lambda)) d \lambda d y=0,
\end{aligned}
$$

uniformly, $0<a \leqq x \leqq b<1$.

We see at once that Theorems VII and VIII together give us the sufficient conditions of Theorem IV and therefore establish the present theorem with regard to the first integral. Since the sector $S^{\prime}$ is arbitrary the arc $\gamma$ may be replaced by $\Gamma$ in the first integral; and then a return to the variable $\lambda$ gives the second integral.

Theorem IX clearly yields a large amount of information concerning the behavior of the expansions arising from two distinct regular differential systems of order $n=2 \mu-1$. There is one point, however, which we desire to examine before stating our final theorems on the comparison of these expansions. If we let $N_{\mathbf{\Lambda}}, \bar{N}_{\Delta}$ be the number of residues of the two Green's functions $G$ and $\bar{G}$ respectively arising from poles in the circle $C$ of radius $\Lambda$, we clearly have $\left|N_{\Delta}-\bar{N}_{\Delta}\right| \leqq K$ independent of $\Lambda$, though it is not necessary that $N_{\Delta}=\bar{N}_{\Delta}$. Thus the expansions determined by the integrals over $C$ are not compared term-by-term in Theorem IX in general. In order to compare the sums given by the first $N$ residues in the two cases we clearly need to obtain a generalization of the theorem of Riemann-Lebesgue, familiar in the theory of Fourier series: we need to show that the sum of any limited number of terms of the expansion becomes arbitrarily small when each term of the sum arises from a pole of $G$ sufficiently removed from the origin. This is the aim of the theorems which succeed.

ThEOREM X. The residues of the Green's function for a regular differential system of order $n=2 \mu-1$ are a set of functions of $x$ and $y$ uniformly bounded for all $x$ and $y$ on $(0,1)$.

On the region $S^{\prime}, n \rho^{n-1} G\left(x, y ; \rho^{n}\right)$ is uniformly bounded for all $x$ and $y$ on $(0,1)$, as we səe from the asymptotic forms discussed in Theorem VII. Now we can compute any desired residue of the Green's function by evaluating the integral 


$$
R_{\nu}(x, y)=\frac{1}{2 \pi i} \int_{\sigma} n \rho^{n-1} G\left(x, y ; \rho^{n}\right) d \rho
$$

taken over the circumference of the circle $\sigma$ of radius $\epsilon$ described about the corresponding pole of $G$ in the region $\Sigma$; the circles $\sigma$ remote from the origin lie entirely interior to a region $S$. Thus we find $\left|R_{\nabla}(x, y)\right| \leqq M \epsilon$ for all $x$ and $y$ on $(0,1), \nu=1,2, \cdots$.

TheOREM XI. Under the hypotheses of Theorem X, the integral

$$
\frac{1}{2 \pi} \int_{\alpha}^{\beta} \int_{\sigma} n \rho^{n-1} G\left(x, y ; \rho^{n}\right) d \rho d y=\int_{\alpha}^{\beta} R_{\nu}(x, y) d y
$$

approaches zero uniformly, $0 \leqq x \leqq 1$, as the center of the circle $\sigma$ recedes indefinitely from the origin. We suppose $0 \leqq \alpha<\beta \leqq 1$.

As in Theorem VIII we divide the integral over $(\alpha, \beta)$ into integrals over $(\alpha, x)$ and $(x, \beta)$, of which we consider only one in detail. We then show that on $S^{\prime}$ we have

It follows that

$$
\int_{x}^{\alpha} n \rho^{n-1} G\left(x, y ; \rho^{n}\right) d y=\frac{m(x, \alpha, \rho)}{\rho} \text {. }
$$

$$
\left|\int_{\sigma} \int_{\alpha}^{\beta} n \rho^{n-1} G\left(x, y ; \rho^{n}\right) d y\right| \leqq \frac{2 \pi M \epsilon}{R^{\prime}}
$$

where $R^{\prime}$ is the shortest distance from the origin to the circle $\sigma$.

Case I. $\rho$ on $S_{1}{ }^{\prime}$. We refer once again to the asymptotic form of $n \rho^{n-1} G\left(x, y ; \rho^{n}\right)$ given in Case I of Theorem VII. We perform the integration with respect to $y$ directly in the determinant $\Delta_{1}$ as in Theorem VIII and find that the contribution of this term is of the form $m(x, \alpha, \rho) / \rho$, just as before. It remains to treat the bracket terms in the asymptotic form. We have

$$
\int_{x}^{\alpha} e^{\rho \omega_{i}(x-y)}\left(\omega_{i}+\frac{m_{i}}{\rho}\right) d y=\frac{1-e^{\rho \omega_{i}(x-\alpha)}}{\rho}+\int_{x}^{\alpha} e^{\rho \omega_{i}(x-y)} \frac{m_{i}}{\rho} d y=\frac{m_{i}(x, \alpha, \rho)}{\rho}
$$

for $\rho$ on $S_{1}{ }^{\prime}, 0 \leqq \alpha \leqq x \leqq 1, i=1, \cdots, \mu$; and

$$
\int_{x}^{\alpha} e^{\rho \omega_{i}(x-y)}\left(\omega_{i}+\frac{m_{i}}{\rho}\right) d y=\frac{m_{i}(x, \alpha, \rho)}{\rho}
$$

for $\rho$ on $S_{1}{ }^{\prime}, 0 \leqq x \leqq \alpha \leqq 1, i=\mu+1, \cdots, n$. Hence, the bracket terms also contribute an expression of the form $m(x, \alpha, \rho) / \rho, \rho$ on $S_{1}{ }^{\prime}$, for all $x$ and $\alpha$ on $(0,1)$. Consequently on $S_{1}{ }^{\prime}$ we have

$$
\int_{x}^{\alpha} n \rho^{n-1} G\left(x, y ; \rho^{n}\right) d y=\frac{m(x, \alpha, \rho)}{\rho} \text {. }
$$


Case II. $\rho$ on $S_{2}{ }^{\prime}$. The procedure is the same as in Case I except for the difference of the two sectors with regard to the constant $\omega_{\mu}$.

As we have already pointed out, the theorem flows at once from the results of Cases I and II.

We now obtain the generalised theorem of Riemann-Lebesgue:

Theorem XII. If the hypotheses of Theorem $\mathrm{X}$ are fulfilled, anc if $f(x)$ is any summable function on $(0,1)$, then

$$
\frac{1}{2 \pi i} \int_{0}^{1} f(y) \int_{\sigma} n \rho^{n-1} G\left(x, y ; \rho^{n}\right) d \rho d y
$$

approaches zero uniformly, $0 \leqq x \leqq 1$, as the center of the circle $\sigma$ recedes indefinitely from the origin. In other words, if $R_{\nu}(x, y)$ is the residue of $G(x, y ; \lambda)$ corresponding to the pole $\lambda=\lambda_{r}$, then

$$
\lim _{\nu \rightarrow \infty} \int_{0}^{1} f(y) R_{\nu}(x, y) d y=0
$$

uniformly, $0 \leqq x \leqq 1$.

The theorem is established at once by comparing Theorems IV, X, XI.

We can now make the following fundamental assertion:*

Theorem XIII. On any closed interval $(a, b)$ completely interior to $(0,1)$ the term-by-term difference of the expansions formed for any summable function $f(x)$ in connection with two regular differential systems of order $n=2 \mu-1$ converges uniformly to zero. These series we shall call Birkhoff series of order $n=2 \mu-1$; and we may restate the preceding result, saying that any two Birkhoff series of order $n=2 \mu-1$ are equivalent on $(a, b)$. In particular, Birkhoff series of order $n=2 \mu-1$ are equivalent to Fourier series on $(a, b)$.

The material on which the proof rests is to be found in Theorems $V$, IX, XII. If we take $l=0$ in Theorem IX we find that

$$
\frac{1}{2 \pi i} \int_{0}^{1} f(y) \int_{C}(G(x, y ; \lambda)-\bar{G}(x, y ; \lambda)) d \lambda d y
$$

gives the difference of $N_{\Delta}$ terms of one series and $\bar{N}_{\Delta}$ of the other, and approaches zero uniformly on $(a, b)$ as $\Lambda \rightarrow \infty$. By Theorem XII we can re-

* Tamarkin, D. E., Chapter V, has shown the truth of the last statement here, without actually discussing the term-by-term difference of the two series; his statement of the result is found in Lemma 6 , where we have $p=1, \omega_{k}(x)=1 / n, q=2 \pi,\left|c_{k}\right|=1$. 
move a limited number of terms from each series without affecting the uniform convergence of their difference to zero, provided that the terms removed correspond to poles of the Green's functions which recede indefinitely from the origin. Because of the regular distribution of the poles of the two Green's functions, we can always do this in such wise that we obtain the term-byterm difference of the first $N$ terms of the two series, which therefore converges uniformly to zero on $(a, b)$ as $N$ becomes infinite, passing through all positive integral values. Theorem $\mathrm{V}$ tells us that the Fourier series is a special case of the Birkhoff series of order $n=2 \mu-1$, and therefore justifies the last statement of the theorem.

The consequences of this theorem are so important that it is worthwhile to describe them in detail. The theorem means essentially that on any interval $(a, b)$ interior to $(0,1)$ any two Birkhoff series of order $n=2 \mu-1$ have exactly the same behavior and both behave like Fourier series. In particular, properties of Fourier series such as uniform and non-uniform convergence, divergence, oscillation, uniform and non-uniform summability, are carried over directly to Birkhoff series. The various tests for the convergence and summability of Fourier series apply also to Birkhoff series. We conclude the existence of continuous functions whose Birkhoff series converge non-uniformly or diverge. Gibbs' phenomenon is common to Fourier series and Birkhoff series. The theorems of Fejér-Chapman and Lebesgue-Hardy are true for Birkhoff series. Another important fact which we may observe is that the behavior of Birkhoff series at any interior point of $(0,1)$ depends only upon the nature of the expanded function in the neighborhood of that point.

It is also in place to note that, for the class of all summable functions, this result is the strongest that can be obtained. The equivalence cannot be extended to the entire interval $(0,1)$ as can be shown by an example.*

In the preceding paragraphs we mentioned the theorems of FejerChapman and Lebesgue-Hardy, which are concerned with the Cesàro summability of Fourier series. It is not without interest to apply our methods to prove these theorems. To accomplish this we need a lemma whose truth is suggested by facts from the theory of Bessel functions.

LEMma VII. The function

$$
\Phi(\alpha)=\int_{-1}^{+1}\left(1-\phi^{4}\right)^{l} e^{-i \alpha \phi} d \phi=2 \int_{0}^{1}\left(1-\phi^{4}\right)^{l} \cos \alpha \phi d \phi, \quad l \geqq 0,
$$

\footnotetext{
* Stone, these Transactions, vol. 26 (1924), pp. 335-355, § II.
} 
satisfies the inequalities

$$
|\Phi(\alpha)| \leqq K, \quad 0 \leqq \alpha \leqq 1 ; \quad|\Phi(\alpha)| \leqq K \alpha^{-l-1}, \quad \alpha \geqq 1,
$$

where $K$ is a suitably chosen positive constant.

The first inequality is obvious; the second we demonstrate by contour integration in the complex $\phi$-plane. We may describe the contour as the boundary of the simply-connected closed region obtained from the rectangle with vertices $-1,+1,+1-\sigma i,-1-\sigma i, \sigma>0$, by the introduction of a cut along the negative axis of imaginaries from $-i$ to $-\sigma i$. In this region the function $\left(1-\phi^{4}\right)^{l} e^{-i \alpha \phi}$ is analytic, except at $-1,-i,+1$, and continuous; at the origin it has the value 1. By Cauchy's theorem we have

$$
\begin{gathered}
\left(\int_{-1}^{+1}+\int_{+1}^{+1-\sigma i}+\int_{1-\sigma i}^{-\sigma i}+\int_{-\sigma i}^{-i}+\int_{-i}^{-\sigma i}+\int_{-\sigma i}^{-1-\sigma i}\right. \\
\left.+\int_{-1-\sigma i}^{-1}\right)\left(1-\phi^{4}\right)^{l} e^{-i \alpha \varphi} d \phi=0 .
\end{gathered}
$$

On writing $\phi=1-i t / \alpha$ we find

$$
\begin{aligned}
\left|\int_{1}^{1-\sigma i}\left(1-\phi^{4}\right)^{l} e^{-i \alpha \phi} d \phi\right| & =\left|\frac{1}{\alpha} \int_{0}^{\sigma \alpha}\left(1-(1-i t / \alpha)^{4}\right)^{l} e^{-i \alpha-t} d t\right| \\
& \leqq \alpha^{-l-1} \int_{0}^{\infty} t^{l}\left(4+6 t+4 t^{2}+t^{3}\right)^{l} e^{-t} d t \leqq K \alpha^{-l-1} / 4,
\end{aligned}
$$

for $\alpha \geqq 1, \sigma>1$. Similarly, if we set $\phi=t-\sigma i$ we obtain

$$
\begin{aligned}
\left|\int_{1-\sigma i}^{-\sigma i}\left(1-\phi^{4}\right)^{l} e^{-i \alpha \phi} d \phi\right| & =\left|\int_{0}^{1}\left(1-(t-\sigma i)^{4}\right)^{l} e^{-\sigma \alpha} e^{-i \alpha t} d t\right| \\
& \leqq e^{-\sigma}\left(1+(1+\sigma)^{4}\right)^{l} \rightarrow 0
\end{aligned}
$$

as $\sigma \rightarrow \infty$, for $\alpha \geqq 1$. Likewise by the substitution $\phi=-i-i t / \alpha$ we see that

$$
\begin{aligned}
\left|\int_{-\sigma i}^{-i}\left(1-\phi^{4}\right)^{l} e^{-i \alpha \phi} d \phi\right| & =\left|\frac{1}{\alpha} \int_{0}^{(\sigma-1) \alpha}\left(1-(1+t / \alpha)^{4}\right)^{l} e^{-\alpha-t} d t\right| \\
& \leqq \alpha^{-l-1} \int_{0}^{\infty} t^{l}\left(4+6 t+4 t^{2}+t^{3}\right)^{l} e^{-t} d t \leqq K \alpha^{-l-1} / 4,
\end{aligned}
$$

for $\alpha \geqq 1, \sigma>1$. Hence, on allowing $\sigma$ to become infinite in these three terms and on treating the last three in the same manner, we find

$$
|\Phi(\alpha)| \leqq K \alpha^{-l-1}, \quad \alpha \geqq 1,
$$

as we were to show. 
We can now establish

TheOREM XIV. The Fourier series for an arbitrary summable function is summable $(C, l), l>0$, to that function almost everywhere.* If the function is continuous, then the summability is uniform, $0<a \leqq x \leqq b<1 . \dagger$

Taking the differential system $u^{\prime}+\lambda u=0, u(0)-u(1)=0$ we are to show that if $f(x)$ is summable

$$
\lim _{\eta \rightarrow \infty} \frac{1}{2 \pi i} \int_{0}^{1} \int_{C_{\nu}} f(y)\left(1-\frac{\lambda^{4}}{\Lambda_{v}^{4}}\right)^{l} G(x, y ; \lambda) d \lambda d y=f(x)
$$

almost everywhere on $(0,1)$. We adopt the notations

$$
F(z)=f(z+x)+f(-z+x)-2 f(x), \mathcal{F}(z)=\int_{0}^{z}|F(z)| d z .
$$

We choose any value of $x$ for which

$$
\lim _{z \rightarrow 0} F(z) / z=0, \quad 0<x<1 .
$$

By a theorem of Lebesgue these values of $x$ form a set of measure one.f Theorems IV, VII, VIII show us that the only significant contributions of the integral are

$$
\begin{aligned}
& \frac{1}{2 \pi i} \int_{0}^{1} \int_{\gamma_{1}} f(y)\left(1-\frac{\lambda^{4}}{\Lambda_{\nu}^{4}}\right)^{l}\left\{e^{\lambda(y-x)} ; 0\right\} d \lambda d y \\
& +\frac{1}{2 \pi i} \int_{0}^{1} \int_{\gamma_{2}} f(y)\left(1-\frac{\lambda^{4}}{\Lambda_{\nu}^{4}}\right)^{l}\left\{0 ; e^{\lambda(y-x)}\right\} d \lambda d y \\
& =\frac{1}{2 \pi} \int_{0}^{x} \int_{-\pi / 2}^{+\pi / 2} f(y)\left(1-e^{4 i \theta}\right)^{l_{\nu} \Lambda_{\nu} e^{\Lambda_{\nu}} e^{i \theta}(y-x)} e^{i \theta} d \theta d y \\
& \quad-\frac{1}{2 \pi} \int_{x}^{1} \int_{\pi / 2}^{3 \pi / 2} f(y)\left(1-e^{4 i \theta}\right)^{l_{\nu}} \Lambda_{\nu} e^{\Lambda_{\nu} e^{i \theta}(y-x)} e^{i \theta} d \theta d y,
\end{aligned}
$$

where we have recalled the fact that $\lambda=\Lambda_{\nu} e^{i \theta}$ on $\gamma$. Henceforth we shall let $\Lambda$ be a continuous variable. In the first integral we set $\phi=-i e^{i \theta}, z=x-y$, and in the second $\phi=-i e^{i \theta}, z=y-x$. There results

$$
\frac{1}{2 \pi} \int_{0}^{x} f(-z+x) \Lambda \Phi(\Lambda z) d z+\frac{1}{2 \pi} \int_{0}^{1-x} f(z+x) \Lambda \Phi(\Lambda z) d z .
$$

\footnotetext{
* Hardy, Proceedings of the London Mathematical Society, ser. 2, vol. 12 (1912), pp. 365-376.

† Chapman, Proceedings of the London Mathematical Society, ser. 2, vol. 9 (1911), pp. 369-409.

‡ Lebesgue, Leçons sur les Séries Trigonométriques, Paris, 1905, \$50, p. 96.
} 
If we take $A \leqq x, 1-x$ we have as $\Lambda \rightarrow \infty$

$$
\begin{gathered}
\left|\int_{A}^{x} f(-z+x) \Lambda \Phi(\Lambda z) d z\right| \leqq K \Lambda^{-l} A^{-l-1} \int_{A}^{x}|f(-z+x)| d z \rightarrow 0, \\
\left|\int_{A}^{1-x} f(z+x) \Lambda \Phi(\Lambda z) d z\right| \leqq K \Lambda^{-l} A^{-l-1} \int_{A}^{1-x}|f(z+x)| d z \rightarrow 0 .
\end{gathered}
$$

Furthermore, we see that

$$
\frac{1}{2 \pi} \int_{0}^{A} \Lambda \Phi(\Lambda z) d z=\frac{1}{\pi} \int_{0}^{1} \int_{0}^{A}\left(1-\phi^{4}\right)^{l} \Lambda \cos \Lambda z \phi d z d \phi=\frac{1}{\pi} \int_{0}^{1}\left(1-\phi^{4}\right)^{i} \frac{\sin \Lambda A \phi}{\phi} d \phi .
$$

The last integral, essentially the well known Dirichlet integral, has the limit $\frac{1}{2}$. To prove our theorem, then, it is sufficient to show that

$$
\lim _{\Lambda \rightarrow \infty} \frac{1}{2 \pi} \int_{0}^{\Lambda} F(z) \Lambda \Phi(\Lambda z) d z=0 .
$$

We can establish this by following Hardy's discussion of a precisely similar integral. In his treatment, the essential properties of the function corresponding to our function $\Phi$ are those which we enumerated in Lemma VII. This theorem was demonstrated by Lebesgue in the case $l=1$, and by Hardy in the more general form. It is not difficult to see that when $0<a \leqq x \leqq b<1$ and $f(x)$ is continuous, the steps of the proof can be carried through in the same manner, with the additional result that the convergence is uniform in $x$ because of the fact that

$$
\lim _{z \rightarrow 0} F(z) / z=0 \text { uniformly, } 0<a \leqq x \leqq b<1 .
$$

This result, due to Fejér for $l=1$, was established in the general case by Chapman.

In our preceding theorems we have compared series only on an interval $(a, b)$ completely interior to the interval $(0,1)$ on which they are defined. If we wish to consider the entire interval $(0,1)$ we find it possible to do so when we compare the series arising from differential systems sufficiently alike. It is sufficient to restrict the boundary conditions so that $\alpha_{1}=\bar{\alpha}_{1}, \cdots$, $\alpha_{n}=\bar{\alpha}_{n}, \beta_{1}=\bar{\beta}_{1}, \cdots, \beta_{n}=\bar{\beta}_{n}, k_{1}=\bar{k}_{1}, \cdots, k_{n}=\bar{k}_{n}$, as we shall see presently. We first introduce new lemmas which serve as useful tools in studying the questions thus raised.

LEMaAa VIII. On any region $S^{\prime}$

$$
\frac{\left[\theta_{0}\right]+e^{\rho \omega_{\mu}}\left[\theta_{1}\right]}{\left[\bar{\theta}_{0}\right]+e^{\rho \omega_{\mu}}\left[\bar{\theta}_{1}\right]}=[1] \text { if } \theta_{0}=\bar{\theta}_{0}, \theta_{1}=\bar{\theta}_{1} \text {. }
$$


On $S_{1}{ }^{\prime}$ we see that

$$
\left(\left[\theta_{0}\right]+e^{\rho \omega_{\mu}}\left[\theta_{1}\right]\right)-\left(\left[\bar{\theta}_{0}\right]+e^{\rho \omega_{\mu}}\left[\bar{\theta}_{1}\right]\right)=[0]+e^{\rho \omega_{\mu}}[0] g=[0],
$$

whence

$$
\frac{\left[\theta_{0}\right]+e^{\rho \omega_{\mu}}\left[\theta_{1}\right]}{\left[\bar{\theta}_{0}\right]+e^{\rho \omega_{\mu}}\left[\bar{\theta}_{1}\right]}=1+\frac{[0]}{\left[\bar{\theta}_{0}\right]+e^{\rho \omega_{\mu}}\left[\bar{\theta}_{1}\right]}=[1] .
$$

Similarly, on $S_{2}{ }^{\prime}$, we find

$$
\frac{\left[\theta_{0}\right]+e^{\rho \omega_{\mu}}\left[\theta_{1}\right]}{\left[\bar{\theta}_{0}\right]+e^{\rho \omega_{\mu}}\left[\bar{\theta}_{1}\right]}=\frac{\left[\theta_{0}\right] e^{-\rho \omega_{\mu}}+\left[\theta_{1}\right]}{\left[\bar{\theta}_{0}\right] e^{-\rho \omega_{\mu}}+\left[\bar{\theta}_{1}\right]}=[1] .
$$

LEMMA IX. The integrals

$$
\begin{array}{lr}
\int_{\gamma_{1}} \int_{\alpha}^{x} e^{\rho \omega_{i}(1-y)} \frac{m(x, y, \rho)}{\rho} d y d \rho & (i=1, \cdots, \mu), \\
\int_{\gamma_{1}} \int_{\alpha}^{x} e^{-\rho \omega_{i} y} \frac{m(x, y, \rho)}{\rho} d y d \rho & (i=\mu+1, \cdots, n), \\
\int_{\gamma_{2}} \int_{\alpha}^{x} e^{\rho \omega_{i}(1-y)} \frac{m(x, y, \rho)}{\rho} d y d \rho & (i=1, \cdots, \mu-1), \\
\int_{\gamma_{2}} \int_{\alpha}^{x} e^{-\rho \omega_{i} y} \frac{m(x, y, \rho)}{\rho} d y d \rho & (i=\mu, \cdots, n),
\end{array}
$$

where $|m(x, y, \rho)| \leqq M$ for all $x$ and $y$ on $(0,1)$ and for all $\rho$ of sufficiently large absolute value on $S_{1}{ }^{\prime}$ or $S_{2}{ }^{\prime}$, as the case may be, converge uniformly to zero as $R \rightarrow \infty, 0 \leqq x \leqq 1,0 \leqq \alpha \leqq 1$.

Leaving aside for the moment the integrals for which $i=\mu$, we may take as typical the following procedure:

$$
\begin{aligned}
\left|\int_{\gamma_{1}} \int_{\alpha}^{x} e^{\rho \omega_{i}(1-y)} \frac{m}{\rho} d y d \rho\right| & \leqq \frac{\pi M}{2 n}\left|\int_{\alpha}^{x} e^{-R \beta(1-y)} d y\right| \\
& =\frac{\pi M}{2 \beta n R}\left|e^{-R \beta(1-x)}-e^{-R \beta(1-\alpha)}\right| \leqq \frac{\pi M}{\beta n R} \rightarrow 0 .
\end{aligned}
$$

In the two integrals where $i=\mu$, we operate as follows:

$$
\begin{aligned}
&\left|\int_{\gamma_{1}} \int_{\alpha}^{x} e^{\rho \omega_{\mu}(1-y)} \frac{m}{\rho} d y d \rho\right| \leqq M\left|\int_{\alpha}^{x} \int_{0}^{\pi / 2 n} e^{-R \phi(1-y) / 2} d \phi d y\right| \\
&=M\left|\int_{\alpha}^{x} \frac{1-e^{-x R(1-y) / 4 n}}{R(1-y)} d y\right| \leqq \frac{M}{R}\left|\int_{(1-\alpha) R}^{(1-x) R} \frac{1-e^{-\pi \xi / 4 n}}{\xi} d \xi\right| \\
& \leqq \frac{2 M}{R} \int_{0}^{R} \frac{1-e^{-x \xi / 4 n}}{\xi} d \xi \rightarrow 0 .
\end{aligned}
$$

The second integral with $i=\mu$ is discussed in an analogous manner. 
We can now prove

THEOREM XV. If $G$ and $\bar{G}$ are the Green's functions for any two regular differential systems of order $n=2 \mu-1$ for which $\alpha_{1}=\bar{\alpha}_{1}, \cdots, \alpha_{n}=\bar{\alpha}_{n}, \beta_{1}=\bar{\beta}_{1}$, $\cdots, \beta_{n}=\bar{\beta}_{n}, k_{1}=\bar{k}_{1}, \cdots, k_{n}=\bar{k}_{n}$, then for any region $S^{\prime}$ the integral

$$
\int_{\gamma} n \rho^{n-1}\left(G\left(x, y ; \rho^{n}\right)-\bar{G}\left(x, y ; \rho^{n}\right)\right) d \rho
$$

is bounded for all $\gamma$ and for all $x$ and $y$ on $(0,1)$.

We must consider separately the integrals over $\boldsymbol{\gamma}_{1}$ and $\boldsymbol{\gamma}_{2}$.

Case I. $\rho$ on $S_{1}{ }^{\prime}$. We refer to the asymptotic forms of $n \rho^{n-1} G$ and $n \rho^{n-1} \bar{G}$ given in Theorem VII. If in the form for $n \rho^{n-1} G$ we expand the determinant $\Delta_{1}$ we are led to a sum of terms each of which is the product of a certain number of exponential functions, bounded for all $\rho$ on $S_{1}$ and for all $x$ and $y$ on $(0,1)$, multiplied by an expression of the type $[A] /\left\{\left[\theta_{0}\right]\right.$ $\left.+e^{\rho \omega_{\mu}}\left[\theta_{1}\right]\right\}$. In $n \rho^{n-1} \bar{G}$ the term containing the corresponding exponentials has the coefficient $[\bar{A}] /\left\{\left[\bar{\theta}_{0}\right]+e^{\rho \omega_{\mu}}\left[\bar{\theta}_{1}\right]\right\}$ where by hypothesis $A=\bar{A}, \theta_{0}=\bar{\theta}_{0}$, $\theta_{1}=\bar{\theta}_{1}$. If we subtract the corresponding terms, the resulting coefficient becomes, by Lemma VIII,

$$
\frac{[A]}{\left[\theta_{0}\right]+e^{\rho \omega_{\mu}}\left[\theta_{1}\right]}-\frac{[\bar{A}]}{\left[\bar{\theta}_{0}\right]+e^{\rho \omega_{\mu}}\left[\bar{\theta}_{1}\right]}=\frac{[A]-[\bar{A}]}{\left[\theta_{0}\right]+e^{\rho \omega_{\mu}}\left[\theta_{1}\right]}=\frac{[0]}{\left[\theta_{0}\right]+e^{\rho \omega_{\mu}}\left[\theta_{1}\right]}=[0]
$$

for all $\rho$ on $S_{1}{ }^{\prime}$ and all $x$ and $y$ on $(0,1)$. Now each product of exponentials from the determinants $\Delta_{1}, \bar{\Delta}_{1}$ contains exactly one of the functions

$$
e^{\rho \omega_{1}(1-y)}, \cdots, e^{\rho \omega_{\mu}(1-y)}, e^{-\rho \omega_{\mu}+1} y, \cdots, e^{-\rho \omega_{n} y} .
$$

If we group the terms in the difference $n \rho^{n-1}(G-\bar{G})$ according to the exponentials in $y$ which they contain, we see that we can write

$$
\begin{aligned}
n \rho^{n-1}(G-\bar{G})= & \left.-\sum_{i=1}^{i=\mu} e^{\rho \omega_{i}(x-y)} \frac{m_{i}(x, y, \rho)}{\rho} ;+\sum_{i=\mu+1}^{i=n} e^{\rho \omega_{i}(x-y)} \frac{m_{i}(x, y, \rho)}{\rho}\right\} \\
& +\sum_{i=1}^{i=\mu} e^{\rho \omega_{i}(1-y)} \frac{m_{n+i}(x, y, \rho)}{\rho}+\sum_{i=\mu+1}^{i=n} e^{-\rho \omega_{i} y} \frac{m_{n+i}(x, y, \rho)}{\rho} \\
= & \frac{m(x, y, \rho)}{\rho},
\end{aligned}
$$

where the functions $m$ are bounded for all $\rho$ on $S_{1}{ }^{\prime}$ and all $x$ and $y$ on $(0,1)$. The application of Lemma III to the last expression proves that

$$
\int_{\gamma_{1}} n \rho^{n-1}\left(G\left(x, y ; \rho^{n}\right)-\bar{G}\left(x, y ; \rho^{n}\right)\right) d \rho
$$

is bounded for all $\gamma_{1}$ on $S_{1}{ }^{\prime}$ and for all $x$ and $y$ on $(0,1)$. 
Case II. $\rho$ on $S_{2}^{\prime}$. We now find for the difference $n \rho^{n-1}(G-\bar{G})$ an expression like that of Case I, different only in having the sums extended from $i=1$ to $i=\mu-1$ and from $i=\mu$ and to $i=n$ respectively. The desired result for the integral over $\gamma_{2}$ is then established at once; and the theorem follows at once.

TheOREM XVI. Under the hypotheses of Theorem XV,

$$
\lim _{R \rightarrow \infty} \int_{\alpha}^{\beta} \int_{\gamma} n \rho^{n-1}\left(G\left(x, y ; \rho^{n}\right)-\bar{G}\left(x, y ; \rho^{n}\right)\right) d \rho d y=0
$$

uniformly for all $x$ on $(0,1)$.

We take the integral over $(\alpha, \beta)$ as the sum of integrals over $(\alpha, x)$ and $(x, \beta)$. Of these we consider but one.

Case I. $\rho$ on $S_{1}{ }^{\prime}$. From the expressions obtained in Theorem $\mathrm{XV}$ we see that

$$
\begin{aligned}
\int_{\alpha}^{x} \int_{\gamma_{1}} n \rho^{n-1}(G-\bar{G}) d \rho d y & \\
= & \int_{\alpha}^{x} \int_{\gamma_{1}}\left\{-\sum_{i=1}^{i=\mu} e^{\rho \omega_{i}(x-y)} \frac{m_{i}}{\rho} ;+\sum_{i=\mu+1}^{i=n} e^{\rho \omega_{i}(x-y)} \frac{m_{i}}{\rho}\right\} d \rho d y \\
& \quad+\int_{\alpha}^{x} \int_{\gamma_{1}}\left(\sum_{i=1}^{i=\mu} e^{\rho \omega_{i}(1-y)} \frac{m_{n+i}}{\rho}+\sum_{i=\mu+1}^{i=n} e^{-\rho \omega_{i} y} \frac{m_{n+i}}{\rho}\right) d \rho d y \rightarrow 0,
\end{aligned}
$$

uniformly, $0 \leqq x \leqq 1,0 \leqq \alpha \leqq 1$, as $R \rightarrow \infty$, by Lemmas VI and IX.

Case II. $\rho$ on $S_{2}{ }^{\prime}$. We use the work of Theorem XV and Lemmas VI and IX exactly as in the preceding case. The double integral over $\gamma$ and $(\alpha, \beta)$ therefore behaves as described.

Theorem XVII. Under the hypotheses of Theorem XV,

$$
\begin{aligned}
& \lim _{R \rightarrow \infty} \int_{0}^{1} f(y) \int_{\gamma} n \rho^{n-1}\left(G\left(x, y ; \rho^{n}\right)-\bar{G}\left(x, y ; \rho^{n}\right)\right) d \rho d y=0, \\
& \lim _{\Delta \rightarrow \infty} \int_{0}^{1} f(y) \int_{C}(G(x, y ; \lambda)-\bar{G}(x, y ; \lambda)) d \lambda d y=0
\end{aligned}
$$

uniformly for all $x$ on $(0,1)$, for any summable function $f(x)$.

We combine Theorems IV, XV, XVI to obtain the limit of the first integral. If we take arcs $\gamma$ on two adjacent sectors $S^{\prime}$ and transfer the results obtained for the integrals over them to the $\lambda$-plane, we find the second limit. 
As Theorems IX and XII resulted in the first part of Theorem XIII, so Theorems XII and XVII lead to

ThEOREM XVIII. The term-by-term difference of two Birkhoff series of order $n=2 \mu-1$ for any summable function $f(x)$ converges uniformly to zero, $0 \leqq x \leqq 1$, provided that the boundary conditions of the two differential systems defining the series can be reduced to normal forms for which $\alpha_{1}=\bar{\alpha}_{1}, \ldots$, $\alpha_{n}=\bar{\alpha}_{n}, \beta_{1}=\bar{\beta}_{1}, \cdots, \beta_{n}=\bar{\beta}_{n}, k_{1}=\bar{k}_{1}, \cdots, k_{n}=\bar{k}_{n}$. Such Birkhoff series are equivalent on $(0,1)$. The differential system

$$
\begin{aligned}
& u^{(n)}+\lambda u=0, \quad n=2 \mu-1, \\
& \alpha_{1} u^{\left(k_{1}\right)}(0)+\beta_{1} u^{\left(k_{1}\right)}(1)=0, \quad n-1 \geqq k_{1} \geqq k_{2} \geqq \cdots \geqq k_{n}, k_{i+2}>k_{i}, \\
& \alpha_{n} u^{\left(k_{n}\right)}(0)+\beta_{n} u^{\left(k_{n}\right)}(1)=0,
\end{aligned}
$$

where $\alpha, \beta, k$ satisfy the conditions for regularity, can therefore be regarded as typical of regular differential systems of order $n=2 \mu-1$ in the consideration of expansion problems with respect to the class of all summable functions.

This theorem is the generalization to the case $n=2 \mu-1$ of the leading results of Haar and Walsh mentioned in the introductory paragraph. We shall apply it to the discussion of Birkhoff series at $x=0$ and $x=1$.

We shall introduce two determinants $\mathscr{D}_{1}(x, y, \rho)$ and $\mathscr{D}_{2}(x, y, \rho)$ which can be obtained from the two determinants $\Delta_{1}$ and $\Delta_{2}$ described under Theorem VII by replacing each term of the form $[A]$ by the dominant constant $A$, and deleting the terms in $e^{\rho \omega_{\mu}}$. We can then prove an interesting intermediate theorem.

TheOREM XIX. If $f(x)$ is any summable function on $(0,1)$ and if $G$ is the Green's function for a regular differential system of order $n=2 \mu-1$, then

$$
\begin{aligned}
\lim _{R \rightarrow \infty} \int_{0}^{1} f(y) \int_{\gamma_{1}}\left(n \rho^{n-1} G\left(x, y ; \rho^{n}\right)\right. & -\left\{-\sum_{i=1}^{i=\mu} e^{\rho \omega_{i}(x-y)} \omega_{i} ;+\sum_{i=\mu+1}^{i=n} e^{\rho \omega_{i}(x-y)} \omega_{i}\right\} \\
& \left.-\frac{1}{\theta_{0}} \mathcal{D}_{1}(x, y, \rho)\right) d \rho d y=0, \\
\lim _{R \rightarrow \infty} \int_{0}^{1} f(y) \int_{\gamma_{2}}\left(n \rho^{n-1} G\left(x, y ; \rho^{n}\right)-\right. & \left\{-\sum_{i=1}^{i=\mu-1} e^{\rho \omega_{i}(x-y)} \omega_{i} ;+\sum_{i=\mu}^{i=n} e^{\rho \omega_{i}(x-y)} \omega_{i}\right\} \\
- & \left.\frac{1}{\theta_{1}} \mathcal{D}_{2}(x, y, \rho)\right) d \rho d y=0,
\end{aligned}
$$

uniformly, $0 \leqq x \leqq 1$. 
By Theorem XVIII, it is sufficient to consider the typical differential system there described.

First integral. On substituting $u_{i}=e^{\rho \omega_{i} x}, v_{i}=-\omega_{i} e^{-\rho \omega_{i} y} / n \rho^{n-1}$ in the explicit formula for the Green's function, we obtain for $\rho$ on $S_{1}{ }^{\prime}$ the asymptotic form given in Theorem VII, where now any expression $[A]$ can be written as the sum of the constant $A$ and a number of exponential terms formed by taking products of $e^{\rho \omega_{1}}, \cdots, e^{\rho \omega_{\mu-1}}, e^{\rho \omega_{\mu}+1}, \cdots, e^{\rho \omega_{n}}$. The expressions $[A]$ in the bracket terms and in the first row of the determinant $\Delta_{1}$ reduce to the constant $A$. Since we can put

$$
\begin{aligned}
e^{\rho \omega_{i}}=e^{\rho \omega_{\mu}} e^{\rho \omega_{i}-\rho \omega_{\mu}},\left|e^{\rho \omega_{i}-\rho \omega_{\mu}}\right|=e^{R\left(\rho \omega_{i}\right)-R\left(\rho \omega_{\mu}\right)} \leqq 1 \quad(i=1, \cdots, \mu-1), \\
e^{-\rho \omega_{i}}=e^{\rho \omega_{\mu}} e^{-\rho \omega_{i}-\rho \omega_{\mu}},\left|e^{-\rho \omega_{i}-\rho \omega_{\mu}}\right|=e^{-R\left(\rho \omega_{i}\right)-R\left(\rho \omega_{\mu}\right)} \leqq 1 \quad(i=\mu+1, \cdots, n),
\end{aligned}
$$

for all $\rho$ on $S_{1}{ }^{\prime}$, we find $[A]=A+e^{\rho \omega_{\mu}} m(\rho)$. If now we expand the determinant $\Delta_{1}$ we see that

$$
\Delta_{1}=\mathcal{D}_{1}(x, y, \rho)+e^{\rho \omega_{\mu}} \sum_{i=1}^{i=\mu} e^{\rho \omega_{i}(1-y)} m_{i}(x, \rho)+e^{\rho \omega_{\mu}} \sum_{i=\mu+1}^{i=n} e^{-\rho \omega_{i} y} m_{i}(x, \rho) .
$$

Slight manipulation shows that

$$
\frac{1}{\left[\theta_{0}\right]+e^{\rho \omega \mu}\left[\theta_{1}\right]}=\frac{1}{\theta_{0}}-\frac{e^{\rho \omega_{\mu m}}(\rho)}{\left[\theta_{0}\right]+e^{\rho \omega \mu}\left[\theta_{1}\right]}=\frac{1}{\theta_{0}}-e^{\rho \omega_{\mu}} M(\rho)
$$

for all $\rho$ on $S_{1}{ }^{\prime}$. Therefore we find

$$
\begin{aligned}
& n \rho^{n-1} G-\left\{-\sum_{i=1}^{i=\mu} e^{\rho \omega_{i}(x-y)} \omega_{i} ;+\sum_{i=\mu+1}^{i=n} e^{\rho \omega_{i}(x-y)} \omega_{i}\right\}-\frac{1}{\theta_{0}} \mathcal{D}_{1} \\
& =e^{\rho \omega_{\mu}}\left(\sum_{i=1}^{i=\mu} c^{\rho \omega_{i}(1-y)} M_{i}(x, \rho)+\sum_{i=\mu+1}^{i=n} e^{-\rho \omega_{i} y} M_{i}(x, \rho)\right)=e^{\rho \omega_{\mu}} M(x, y, \rho)
\end{aligned}
$$

where the functions $M$ are uniformly bounded for all $x$ and $y$ on $(0,1)$ and for all $\rho$ on $S_{1}{ }^{\prime}$. The first integral can now be brought under Theorem IV. For

$$
\left|\int_{\gamma_{1}} e^{\rho \omega_{\mu}} M(x, y, \rho) d \rho\right| \leqq M \int_{0}^{\pi / 2 n} e^{-R \phi / 2} R d \phi=2 M\left(1-e^{-\pi R / 4 n}\right) \leqq 2 M,
$$

while

$$
\begin{gathered}
\left|\int_{\alpha}^{\beta} \int_{\gamma_{1}} e^{\rho \omega_{\mu}}\left(\sum_{i=1}^{i=\mu} e^{\rho \omega_{i}(1-y)} M_{i}(x, \rho)+\sum_{i=\mu+1}^{i=n} e^{-\rho \omega_{i} \nu} M_{i}(x, \rho)\right) d \rho d y\right| \\
=\left|\int_{\gamma_{1}} e^{\rho \omega_{\mu}}\left(\sum_{i=1}^{i=\mu} M_{i} \frac{e^{\rho \omega_{i}(1-\alpha)}-e^{\rho \omega_{i}(1-\beta)}}{\omega_{i}}+\sum_{i=\mu+1}^{i=n} M_{i} \frac{e^{-\rho \omega_{i} \alpha}-e^{-\rho \omega_{i} \beta}}{\omega_{i}}\right) \frac{d \rho}{\rho}\right| \\
\leqq M^{\prime} \int_{0}^{\pi / 2 n} e^{-R \phi / 2} d \phi \rightarrow 0, \quad 0 \leqq x \leqq 1
\end{gathered}
$$

The theorem is therefore established for the first integral. 
Second integral. By proceeding as in the case of the first integral, we show that the integrand in the second has entirely similar properties, due account being taken of the behavior of $\rho \omega_{\mu}$ on $S_{2}{ }^{\prime}$. The theorem is then easily proved for this integral.

In these facts, then, we see the expansion problem set by Birkhoff stripped of all asymptotic encumbrances and expressed explicitly as a problem involving only the elementary functions in linear combination.

On setting $x=0, x=1$, in the integrals of the preceding theorem we can effect still further reductions. We denote by $D_{1}{ }^{0}, D_{1}{ }^{1}, D_{2}{ }^{0}, D_{2}{ }^{1}$ determinants obtained from $\mathscr{D}_{1}(0, y, \rho), \mathscr{D}_{1}(1, y, \rho), \mathscr{D}_{2}(0, y, \rho), \mathscr{D}_{2}(1, y, \rho)$ respectively by appropriate modifications of the first rows; more exactly, in the last four determinants those terms of the first rows explicitly involving the variable $\rho$ as an exponential are replaced by zeros.

ThEOREM XX. The limits described in Theorem XIX are still valid when $x$ is set equal to 0 or 1 and the determinants $D$ are replaced by the corresponding determinants $D$. For example,

$$
\lim _{R \rightarrow \infty} \int_{0}^{1} f(y) \int_{\gamma_{1}}\left(n \rho^{n-1} G\left(0, y ; \rho^{n}\right)-\sum_{i=\mu+1}^{i=n} e^{-\rho \omega_{i} y_{\omega_{i}}}-\frac{1}{\theta_{0}} D_{1}^{0}(y, \rho)\right) d \rho d y=0 .
$$

By Theorem XIX it is sufficient to show that

$$
\lim _{R \rightarrow \infty} \int_{0}^{1} f(y) \int_{\gamma_{1}}\left(\mathcal{D}_{i}(k, y, \rho)-D_{i}^{k}(y, \rho)\right) d \rho d y=0
$$

for $i=1,2$ and $k=0,1$. Since we have, by reasoning like that used in the proof of Theorem XIX,

$$
\mathcal{D}_{1}(k, y, \rho)-D_{1}^{k}(y, \rho)=e^{\rho \mu_{\mu} m_{k}}(y, \rho)
$$

where the functions $m$ are uniformly bounded, $0 \leqq y \leqq 1$, for $\rho$ on $S_{1}, k=0$, 1 ; and since we can obtain similar expressions for the difference $\mathcal{D}_{2}(k, y, \rho)$ $-D_{2}{ }^{k}(y, \rho)$ for $k=0,1$, we are able to complete the proof exactly as in Theorem XIX.

To apply Theorem XX to the study of the convergence of Birkhoff series at $x=0$ and $x=1$ we demonstrate the three lemmas which follow. These lemmas are generalisations of standard theorems concerning the Dirichlet integral.

LEMмA X. If $f(x)$ is summable on $(0,1)$ and if $\lambda_{1}=e^{i \theta_{1}}$, and $\lambda=e_{2}{ }^{i \theta_{2}}$, where $\theta_{1}$ and $\theta_{2}$ are two distinct constants on the range $\left(-\frac{1}{2} \pi, \frac{1}{2} \pi\right)$, then

$$
\lim _{R \rightarrow \infty} \int_{e}^{1} f(y) \frac{e^{-R \lambda_{1} y}-e^{-R \lambda_{2} y}}{y} d y=0, \quad \epsilon>0 .
$$


Since the first factor of the integrand, $f(y) / y$, is summable on $(\epsilon, 1)$ and since the second, $e^{-R \lambda_{1} y}-e^{-R \lambda_{y} y}$, satisfies the sufficient conditions of Theorem IV, as may easily be shown, the lemma is apparent.

LEMMA XI. If $\phi(x)$ is summable on $(0,1)$ and of bounded variation on $(0, \epsilon)$, then

$$
\lim _{R \rightarrow \infty} \int_{0}^{1} \phi(y) \frac{e^{-R \lambda_{1} y}-e^{-R \lambda_{2} y}}{y} d y=\phi(+0)\left(\theta_{2}-\theta_{1}\right) i .
$$

We show that the function $\left(e^{-R \lambda_{1} y}-e^{-R \lambda_{y} y}\right) / y$ has two properties which are the essential elements in well known proofs of this lemma in the case of the Dirichlet integral.

In the first place, we show that

$$
\lim _{R \rightarrow \infty} \int_{0}^{1} \frac{e^{-R \lambda_{1} y}-e^{-R \lambda_{2} y}}{y} d y=\left(\theta_{2}-\theta_{1}\right) i
$$

In the plane of the complex variable $t$ we determine a contour consisting of the rectilinear segments $\left(r \lambda_{1}, R \lambda_{1}\right)$ and $\left(r \lambda_{2}, R \lambda_{2}\right)$ and two circular arcs $c$ and $C$ of radii $r$ and $R$ respectively described about the origin and lying on the right half-plane. Then

$$
\lim _{R \rightarrow \infty} \int_{0}^{1}\left(e^{-R \lambda_{1} y}-e^{-R \lambda_{2} y}\right) \frac{d y}{y}=\lim _{R \rightarrow \infty} \lim _{r \rightarrow 0}\left(\int_{r \lambda_{1}}^{R \lambda_{1}} \frac{e^{-t}}{t} d t-\int_{r \lambda_{1}}^{R \lambda_{2}} \frac{e^{-t}}{t} d t\right) .
$$

By using the contour described and applying Cauchy's theorem, we can replace the last limit by

$$
\lim _{r \rightarrow \infty} \int_{c} \frac{e^{-t}}{t} d t-\lim _{R \rightarrow \infty} \int_{C} \frac{e^{-t}}{t} d t=\left(\theta_{2}-\theta_{1}\right) i-\lim _{R \rightarrow \infty} \int_{C} \frac{e^{-t}}{t} d t=\left(\theta_{2}-\theta_{1}\right) i .
$$

The second property needed is that the integral

$$
\int_{a}^{b} \frac{e^{-R \lambda_{1} y}-e^{-R \lambda_{2} y}}{y} d y
$$

is a uniformly bounded function of $R$ for all $a$ and $b$ on $(0,1)$, no matter how $a$ and $b$ may vary with $R$. Clearly it is sufficient to show this for the special case $a=0$. On writing $B=R b$, we find

$$
\int_{0}^{b} \frac{e^{-R \lambda_{1} y}-e^{-R \lambda_{2} y}}{y} d y=\left(\theta_{2}-\theta_{1}\right) i-\int_{B \lambda_{1}}^{B \lambda_{2}} \frac{e^{-t} d t}{t},
$$

whence the result is apparent. 
It is now a simple matter to demonstrate the lemma.*

LEMma XII. If $\phi(x)$ is summable on $(0,1)$ and if

$$
\Phi(x)=\frac{1}{x} \int_{0}^{x} \phi(x) d x
$$

is of bounded variation on $(0, \epsilon)$, then

$$
\lim _{R \rightarrow \infty} \int_{0}^{1} \phi(y) \frac{e^{-R \lambda_{1} y}-e^{-R \lambda_{2} y}}{y} d y=\Phi(+0)\left(\theta_{2}-\theta_{1}\right) i .
$$

The proof can be carried out along the lines indicated by de la Vallee Poussin in a paper in which he established this result for the Dirichlet integral. $\dagger$ As he points out, this lemma includes Lemma XII as a special case.

We next prove

ThEOREM XXI. If $f(x)$ is summable on $(0,1)$, then the convergence at $x=0$ or at $x=1$ of the Birkhoff series of order $n=2 \mu-1$ for this function is independent of the nature of $f(x)$ outside arbitrarily small neighborhoods of the points $x=0$ and $x=1$.

By Theorem XX the convergence of the series in question depends upon the behavior of certain integrals which we can write out explicitly and reduce to the type discussed in Lemmas X-XII. These integrals arise from the last columns of the determinants $D$. We use $\lambda_{1}, \lambda_{2}, \theta_{1}, \theta_{2}$ to denote any constants satisfying the relations laid down in Lemma X. Then, by fundamental properties of $\rho \omega_{1}, \cdots, \rho \omega_{n}$ on $S_{1}, S_{2}$, we find that the integrals in question can be put in the forms

$$
\begin{aligned}
\int_{0}^{1} f(y) \int_{\gamma_{1}} \omega_{k} e^{\rho \omega_{k}(1-y)} d \rho d y & =\int_{0}^{1} f(1-y) \int_{\gamma_{1}} \omega_{k} e^{\rho \omega_{k} \nu} d \rho d y \\
& =\int_{0}^{1} f(1-y) \frac{e^{-R \lambda_{1} y}-e^{-R \lambda_{2} y}}{y} d y
\end{aligned}
$$

where $\theta_{2}-\theta_{1}=-\pi / 2 n, k=1, \cdots, \mu$;

$$
\int_{0}^{1} f(y) \int_{\gamma_{1}} \omega_{k} e^{-\rho \omega_{k} y} d \rho d y=\int_{0}^{1} f(y) \frac{e^{-R \lambda_{1} y}-e^{-R \lambda_{2} y}}{y} d y
$$

* Hobson, The Theory of Functions of a Real Variable, Cambridge, 1907; ed. 1, $\$ 450$.

† de la Vallee Poussin, Rendiconti del Circolo Matematico di Palermo, vol. 31 (1911), pp. 296-299. 
where $\theta_{2}-\theta_{1}=+\pi / 2 n, k=\mu+1, \cdots, n$;

$$
\int_{0}^{1} f(y) \int_{\gamma_{2}} \omega_{k} e^{\rho \omega_{k}(1-y)} d \rho d y=\int_{0}^{1} f(1-y) \frac{e^{-R \lambda_{1} y}-e^{-R \lambda_{2} y}}{y} d y
$$

where $\theta_{2}-\theta_{1}=-\pi / 2 n, k=1, \cdots, \mu-1$;

$$
\int_{0}^{1} f(y) \int_{\gamma_{2}} \omega_{k} e^{-\rho \omega_{k} y} d \rho d y=\int_{0}^{1} f(y) \frac{e^{-R \lambda_{1} y}-e^{-R \lambda_{2} y}}{y} d y
$$

where $\theta_{2}-\theta_{1}=+\pi / 2 n, k=\mu, \cdots, n$. It is immediately evident from Lemma $\mathbf{X}$ that the behavior of these integrals, and therefore of the series for $f(x)$ at $x=0$ or at $x=1$, is independent of the nature of $f(x)$ except near these points.

The use of the integrals computed in the preceding paragraph and of Lemma XII enables us to state

TheOREM XXII. If $\phi(x)$ is summable on $(0,1)$, and if

$$
\Phi_{1}(x)=\frac{1}{x} \int_{0}^{x} \phi(x) d x, \quad \Phi_{2}(x)=\frac{1}{x} \int_{0}^{x} \phi(1-x) d x
$$

are of bounded variation near $x=0$, then

$$
\lim _{R \rightarrow \infty} \frac{1}{2 \pi \sqrt{-1}} \int_{0}^{1} \phi(y) \int_{\gamma_{i}} n \rho^{n-1} G\left(k, y ; \rho^{n}\right) d \rho d y=A_{i k} \Phi_{1}(+0)+B_{i k} \Phi_{2}(+0)
$$

where $A_{i k}$ and $B_{i k}$ are constants depending only upon the boundary conditions, for $i=1,2, k=0,1$. For example,

$$
\begin{aligned}
& A_{10} \Phi_{1}(+0)+B_{10} \Phi_{2}(+0) \equiv \frac{\mu-1}{4 n} \Phi_{1}(+0)
\end{aligned}
$$

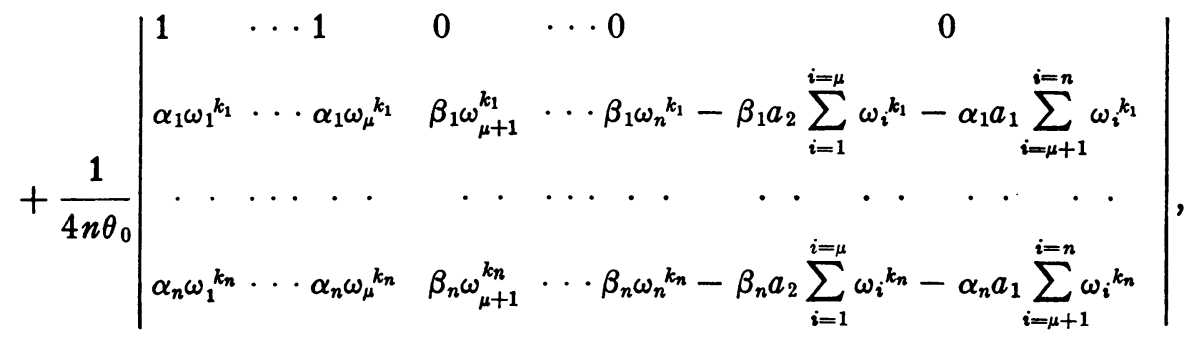

where $a_{1}=\Phi_{1}(+0), a_{2}=\Phi_{2}(+0)$. The other constants may be explicitly determined in the same manner by reference to Theorem $\mathrm{XX}$ and the forms of the determinants $D$. 
This theorem is a generalization of the result announced by Birkhoff for the case $n=2 \mu^{*}$.

We shall make no further applications of Theorem XX. In Theorem IV the sufficient conditions given are also necessary; by combining them with Theorem XX we find necessary conditions that

$$
\lim _{\Lambda \rightarrow \infty} \int_{0}^{1} \int_{C} f(y)(G(x, y ; \lambda)-\bar{G}(x, y ; \lambda)) d \lambda d y=0
$$

uniformly, $0 \leqq x \leqq 1$. It seems as though these conditions in their simplest form should involve only the constants $\alpha, \bar{\alpha}, \beta, \bar{\beta}, k, \bar{k}$, but we have been unable to ascertain the accuracy of this conjecture.

\section{ThE ADJOINT SERIES FOR $n=2 \mu-1$}

We shall next take up the expansion problem which comes to us from the integral

$$
\frac{1}{2 \pi i} \int_{0}^{1} f(y) \int_{C_{\nu}} G(y, x ; \lambda) d \lambda d y,
$$

where $G(x, y ; \lambda)$ is the Green's function associated with a regular differential system of order $n=2 \mu-1$. If this differential system admits an adjoint, in which the parameter appears as $-\lambda$ instead of $+\lambda$, the Green's function for this adjoint system is precisely $G(y, x ; \lambda)$. Since the adjoint system is essentially a regular differential system of order $n=2 \mu-1$ of the type discussed in $\S I V$, the work of that section can be applied directly. Since the differential systems which we are discussing are too general to admit adjoints except in restricted cases, we are faced with a new expansion problem. The series obtained we call the adjoint series of order $n=2 \mu-1$, for obvious reasons. Fortunately it is possible to throw the problem into a form which presents such similarities to that discussed in §IV that we do not need to do more than paraphrase the principal theorems demonstrated hitherto.

In fact, if we make the substitutions $\bar{x}=1-x, \bar{y}=1-y, \bar{f}(\bar{y})=f(1-\bar{y})$, we find at once

$$
\int_{0}^{1} f(y) \int_{C_{v}} G(y, x ; \lambda) d \lambda d y=\int_{0}^{1} \bar{f}(\bar{y}) \int_{C_{\nu}} G(1-\bar{y}, 1-\bar{x} ; \lambda) d \lambda d \bar{y} .
$$

Then, by interchanging $x$ and $y$ in the asymptotic forms of Theorem VII and subsequently making the substitutions described, we obtain asymptotic

* Birkhoff, Rendiconti del Circolo Matematico di Palermo, vol. 36 (1913), pp. 125-126. 
forms for $n \rho^{n-1} G\left(1-\bar{y}, 1-\bar{x} ; \rho^{n}\right)$ which are to all intents and purposes the same as those studied in the preceding section. The fact that in the determinants involved the terms in $x$ and $y$ occur in the last column and the first row respectively instead of vice versa is entirely unessential. Thus for the difference $G(1-\bar{y}, 1-\bar{x} ; \lambda)-\bar{G}(1-\bar{y}, 1-\bar{x} ; \lambda)$ where $G(x, y ; \lambda)$ and $\bar{G}(x, y ; \lambda)$ are the Green's functions for two regular differential systems of order $n=2 \mu-1$ we can state theorems analogous to Theorems IX and XVII. For each Green's function there is a theorem parallel to Theorem XII. Then we find the analogues of Theorems XIII and XVIII. Theorems XIX, $\mathrm{XX}, \mathrm{XXI}$ and XXII likewise have images in the present case, not only by reasoning parallel to that of $\S \mathrm{IV}$, but also by the fact that the Green's function can at this stage be regarded as arising from the typical differential system of Theorem XVIII, which admits an adjoint falling under §IV. When these theorems have been phrased in terms of the variables $\bar{x}$ and $\bar{y}$, it is easy to return to the variables $x$ and $y$.

Thus we have

TheOrem XXIII. If $f(x)$ is summable on $(0,1)$

$$
\lim _{\Lambda \rightarrow \infty} \int_{0}^{1} f(y) \int_{C}(G(y, x ; \lambda)-\bar{G}(y, x ; \lambda)) d \lambda d y=0
$$

uniformly, $0<a \leqq x \leqq b<1$.

TheOREM XXIV. If $f(x)$ is summable on $(0,1)$ and if $R_{\nu}(y, x)$ is the residue of $G(y, x ; \lambda)$ at $\lambda=\lambda_{\text {, }}$, then

$$
\lim _{n \rightarrow \infty} \int_{0}^{1} f(y) R_{\nabla}(y, x) d y=0
$$

uniformly, $0 \leqq x \leqq 1$.

THEOREM XXV. On any closed interval $(a, b)$ completely interior to $(0,1)$ the term-by-term difference of the adjoint series formed for any summable function $f(x)$ in connection with two regular differential systems of order $n=2 \mu-1$ converges uniformly to zero. We say that these adjoint series are equivalent on $(a, b)$; in particular, they are equivalent to Fourier series on $(a, b)$

The last remark results from the fact that the Fourier series obtained as special Birkhoff series in $\S$ III are not altered by an interchange of $x$ and $y$. 
TheOREM XXVI. The Green's functions associated with two regular differential systems of order $n=2 \mu-1$ for which $\alpha_{1}=\bar{\alpha}_{1}, \cdots, \alpha_{n}=\bar{\alpha}_{n}, \beta_{1}=\bar{\beta}_{1}$, $\cdots, \beta_{n}=\bar{\beta}_{n}, k_{1}=\bar{k}_{1}, \cdots, k_{n}=\bar{k}_{n}$, are such that for any summable function $f(x)$

$$
\lim _{\Delta \rightarrow \infty} \int_{0}^{1} f(y) \int_{C}(G(y, x ; \lambda)-\bar{G}(y, x ; \lambda)) d \lambda d y=0
$$

uniformly, $0 \leqq x \leqq 1$.

TheOReM XXVII. On the interval $(0,1)$ the term-by-term difference of the adjoint series formed for any summable function $f(x)$ in connection with two regular differential systems of the form described in Theorem XXVI converges uniformly to zero. Such adjoint series are equivalent on $(0,1)$. The adjoint series arising from the regular differential system

$$
\begin{array}{cl}
u^{(n)}+\lambda u=0, & n=2 \mu-1, \\
\alpha_{1} u^{\left(k_{1}\right)}(0)+\beta_{1} u^{\left(k_{1}\right)}(1)=0, & n-1 \geqq k_{1} \geqq k_{2} \geqq \cdots \geqq k_{n}, k_{i+2}>k_{i}, \\
\cdot \cdot \cdot \cdot \cdot \cdot \cdot & \cdot \cdot \cdot \\
\alpha_{n} u^{\left(k_{n}\right)}(0)+\beta_{n} u^{\left(k_{n}\right)}(1)=0 &
\end{array}
$$

may be regarded as typical, so far as the class of all summable functions is concerned.

Theorem XXVIII. If $f(x)$ is summable on $(0,1)$ then

$$
\begin{gathered}
\lim _{R \rightarrow \infty} \int_{0}^{1} f(y) \int_{\gamma_{1}}\left(n \rho^{n-1} G\left(y, x ; \rho^{n}\right)-\left\{\sum_{i=\mu+1}^{i=n} e^{\rho \omega_{i}(y-x)} \omega_{i} ;-\sum_{i=1}^{i-\mu} e^{\rho \omega_{i}(y-x)} \omega_{i}\right\}\right. \\
\left.-\frac{1}{\theta_{0}} \mathscr{D}_{1}(y, x, \rho)\right) d \rho d y=0, \\
\lim _{R \rightarrow \infty} \int_{0}^{1} f(y) \int_{\gamma_{2}}\left(n \rho^{n-1} G\left(y, x ; \rho^{n}\right)-\left\{\sum_{i=\mu}^{i=n} e^{\rho \omega_{i}(y-x)} \omega_{i} ;-\sum_{i=1}^{i-\mu-1} e^{\rho \omega_{i}(y-z)} \omega_{i}\right\}\right. \\
\left.-\frac{1}{\theta_{1}} \mathcal{D}_{2}(y, x, \rho)\right) d \rho d y=0,
\end{gathered}
$$

uniformly, $0 \leqq x \leqq 1$.

We now obtain from the four determinants $\mathscr{D}_{1}(y, 0, \rho), \Phi_{1}(y, 1, \rho)$, $\mathscr{D}_{2}(y, 0, \rho), \mathscr{D}_{2}(y, 1, \rho)$ respectively four new determinants $\mathfrak{D}_{1}^{0}, \mathfrak{D}_{1}^{1}, \mathfrak{D}_{2}{ }^{0}, \mathfrak{D}_{2}{ }^{1}$ analogous to the determinants $D$ introduced in Theorem XX. In any determinant $D$ the terms in the last column explicitly involving the variable $\rho$ are replaced by zero to give the corresponding determinant $\mathfrak{D}$. 
TheOrem XXIX. If $f(x)$ is summable on $(0,1)$ the limits described in Theorem XXVIII are valid when $x$ is set equal to 0 or 1 and the determinants $\Phi$ are replaced by the corresponding determinants $\mathfrak{D}$. For example,

$$
\lim _{R \rightarrow \infty} \int_{0}^{1} f(y) \int_{\gamma_{1}}\left(n \rho^{n-1} G\left(y, 0 ; \rho^{n}\right)+\sum_{i=1}^{i=\mu} e^{\rho \omega i} \omega_{\omega_{i}}-\frac{1}{\theta_{0}} \mathfrak{D}_{1}{ }^{0}(y, \rho)\right) d \rho d y=0 .
$$

ThEOREM XXX. If $f(x)$ is summable on $(0,1)$, then the convergence at $x=0$ or at $x=1$ of the formal adjoint series of order $n=2 \mu-1$ for this function is independent of the nature of $f(x)$ outside arbitrarily small neighborhoods of the points $x=0$ and $x=1$.

Theorem XXXI. If $\phi(x)$ is summable on $(0,1)$ and if

$$
\Phi_{1}(x)=\frac{1}{x} \int_{0}^{x} \phi(x) d x, \quad \Phi_{2}(x)=\frac{1}{x} \int_{0}^{x} \phi(1-x) d x
$$

are of bounded variation near $x=0$, then

$$
\lim _{R \rightarrow \infty} \frac{1}{2 \pi \sqrt{-1}} \int_{0}^{1} \phi(y) \int_{\gamma_{i}}^{1} n \rho^{n-1} G\left(y, k ; \rho^{n}\right) d \rho d y=\mathfrak{A}_{i k} \Phi_{1}(+0)+\mathfrak{B}_{i k} \Phi_{2}(+0)
$$

where the constants $\mathfrak{A}$ and $\mathfrak{B}$ depend only on the boundary conditions, $i=1,2$, $k=0,1$. In particular,

$$
\begin{aligned}
& \mathfrak{A}_{10} \Phi_{1}(+0)+\mathfrak{B}_{10} \Phi_{2}(+0) \equiv \frac{\mu}{4 n} \Phi_{1}(+0)
\end{aligned}
$$

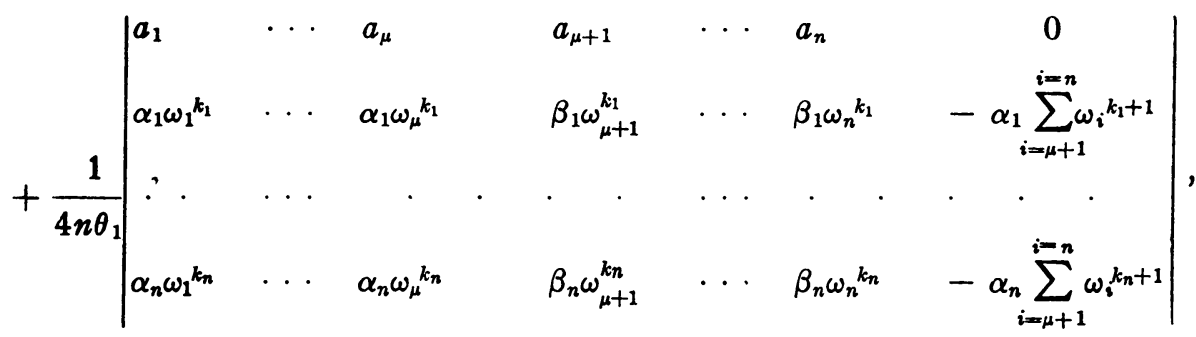

where $a_{i}=-\Phi_{1}(+0) / \omega_{i}, i=1,2, \cdots, \mu$, and $a_{i}=\Phi_{2}(+0) / \omega_{i}, i=\mu+1, \cdots, n$. The other constants may be expressed similarly by reference to Theorem XXIX and the explicit forms of the determinants $D$.

This completes our study of the adjoint series of order $n=2 \mu-1$.

VI. The DERIVEd SERIES OF BIRKHOFF, $n=2 \mu-1$

It is now convenient to restrict our attention to the regular differential system 


$$
\begin{aligned}
& u^{(n)}+\star+p_{2} u^{(n-2)}+\cdots+\left(p_{n}+\lambda\right) u=0, \\
& W_{1}(u)=0, \cdots, W_{n}(u)=0,
\end{aligned}
$$

where $p_{2}, \cdots, p_{n}$ are real or complex functions of the real variable $x$, continuous together with their derivatives of all orders on $(0,1)$. The derived series of Birkhoff are then expressed as the integral

$$
\frac{1}{2 \pi i} \frac{\partial^{k}}{\partial x^{k}} \int_{0}^{1} f(y) \int_{C_{\nu}} G(x, y ; \lambda) d \lambda d y \quad(k=1,2, \cdots)
$$

while a method of summing these series is given by

$$
\frac{1}{2 \pi i} \frac{\partial^{k}}{\partial x^{k}} \int_{0}^{1} f(y) \int_{C_{\nu}}\left(1-\frac{\lambda^{4}}{\Lambda_{\nu}^{4}}\right)^{k+l} G(x, y ; \lambda) d \lambda d y, \quad l \geqq 0 .
$$

If we wish to consider the series only for $k=1,2, \cdots, K$, the functions $p_{2}, \cdots, p_{n}$ do not have to be so heavily restricted; but the complications in statement thus introduced do not yield a proportionate increase in interest.

Lemma XIII. Under the present hypotheses

$$
\begin{aligned}
& \frac{\partial^{k}}{\partial x^{k}} \int_{0}^{1} f(y) \int_{C_{\nu}}\left(1-\frac{\lambda^{4}}{\Lambda_{\nu}^{4}}\right)^{k+l} G(x, y ; \lambda) d \lambda d y \\
& \quad=\int_{0}^{x} f(y) \int_{C_{\nu}}\left(1-\frac{\lambda^{4}}{\Lambda_{\nu}^{4}}\right)^{k+l} \frac{\partial^{k}}{\partial x^{k}} G(x, y ; \lambda) d \lambda d y \\
& \quad+\int_{x}^{1} f(y) \int_{C_{\nu}}\left(1-\frac{\lambda^{4}}{\Lambda_{\nu}^{4}}\right)^{k+l} \frac{\partial^{k}}{\partial x^{k}} G(x, y ; \lambda) d \lambda d y
\end{aligned}
$$

Since

$$
\int_{C_{\nu}}\left(1-\frac{\lambda^{4}}{\Lambda_{\nu}^{4}}\right)^{k+l} G(x, y ; \lambda) d \lambda
$$

is continuous together with its partial derivatives of all orders with respect to $x$ for all $x$ and $y$ on $(0,1)$ we have

$$
\frac{\partial^{k}}{\partial x^{k}} \int_{0}^{1} f(y) \int_{C_{\nu}}\left(1-\frac{\lambda^{4}}{\Lambda_{\nu}^{4}}\right)^{k+l} G d \lambda d y=\int_{0}^{1} f(y) \frac{\partial^{k}}{\partial x^{k}} \int_{C_{\nu}}\left(1-\frac{\lambda^{4}}{\Lambda_{\nu}^{4}}\right)^{k+l} G d \lambda d y .
$$

Now on the ranges $0 \leqq y<x, 0 \leqq x \leqq 1$, and $x<y \leqq 1,0 \leqq x \leqq 1, G(x, y ; \lambda)$ is continuous together with its partial derivatives of all orders with respect to $x$. Hence, on each of these ranges

$$
\frac{\partial^{k}}{\partial x^{k}} \int_{C_{\nu}}\left(1-\frac{\lambda^{4}}{\Lambda_{\nu}^{4}}\right)^{k+l} G d \lambda d y=\int_{C_{\nu}}\left(1-\frac{\lambda^{4}}{\Lambda_{\nu}^{4}}\right)^{k+l} \frac{\partial^{k}}{\partial x^{k}} G d \lambda d y .
$$


The lemma follows at once.

LEMaA XIV. The functions $v_{1}(y), \cdots, v_{n}(y)$ defined by the identity

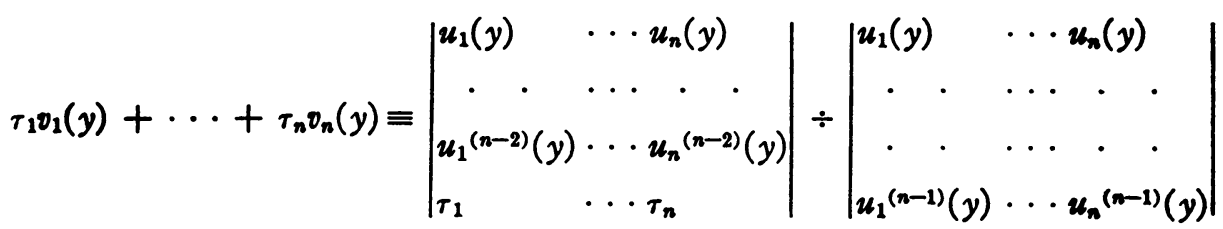
have for a given sector $S$ the asymptotic forms

$$
v_{i}(y) \equiv \frac{-\omega_{i}}{n \rho^{n-1}} e^{-\rho \omega_{i y}}\left(1+\sum_{l=1}^{l-m} \frac{B_{l}(y)}{\left(\rho \omega_{i}\right)^{l}}+\frac{E_{i}(y, \rho)}{\rho^{m+1}}\right),
$$

$i=1, \cdots, n$, where the functions $B_{l}(y), l=1, \cdots, m$, are continuous together with their derivatives of all orders on $(0,1)$, and the functions $E$ are uniformly bounded for all $y$ on $(0,1)$ and for all $\rho$ of sufficiently great absolute value on $S$. The functions $B_{l}(y)$ are independent of the sector $S .^{*}$

We refer to Theorem III', from which we obtain

$$
u_{i}^{(k)}(y)=\left(\rho \omega_{i}\right)^{k} e^{\rho \omega_{i y}} P_{k}\left(\rho \omega_{i}\right)+\left(\rho \omega_{i}\right)^{k} e^{\rho \omega_{i} y} \frac{E_{i k}(y, \rho)}{\rho^{m+1}}
$$

where $P_{k}(z)$ is a polynomial in $1 / z$, with coefficients which are functions of $y$ and with the constant term 1 . The denominator determinant in the defining expression for the functions $v_{1}(y), \cdots, v_{n}(y)$ is known to be independent of y. Hence we have

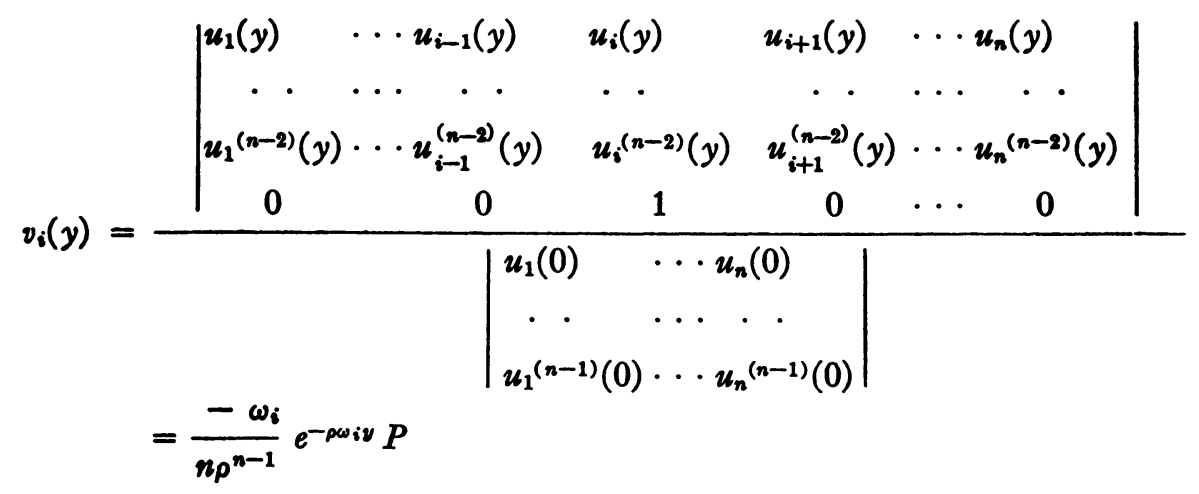

- Milne, Bulletin of the American Mathematical Society, vol. 23 (1916-17), pp. 166-169. 
where $P$ is the quotient

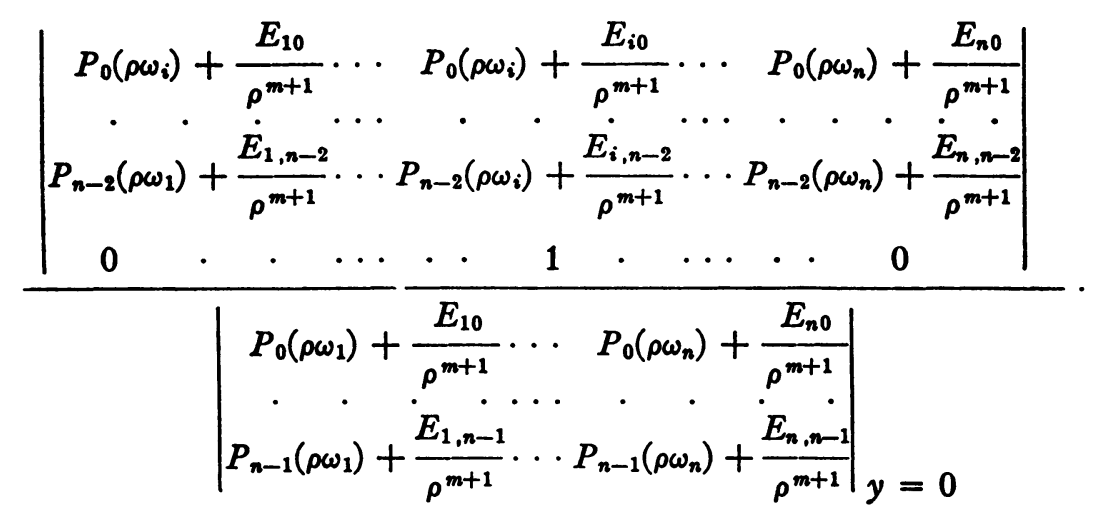

On expanding the determinants and performing the indicated division by the usual processes employed in dealing with asymptotic forms we find

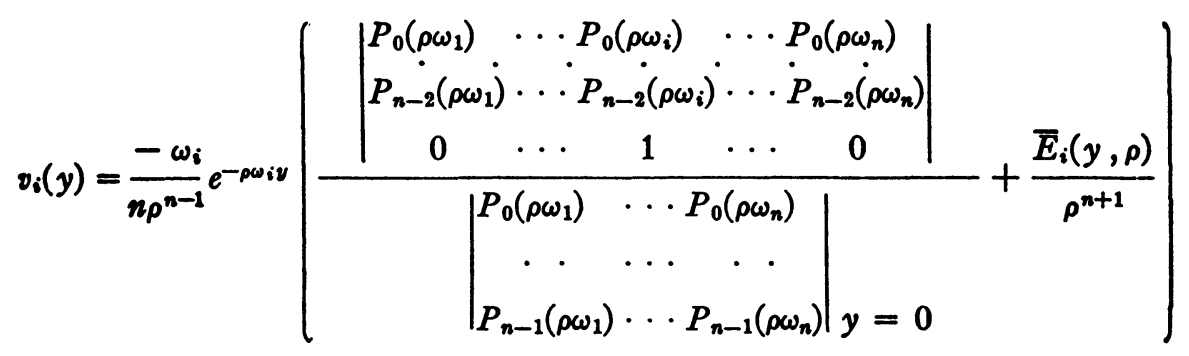

$$
\begin{aligned}
& =\bar{v}_{i}(y)+\frac{-\omega_{i}}{n \rho^{n-1}} e^{-\rho \omega_{i} y} \frac{\bar{E}_{i}(y, \rho)}{\rho^{m+1}} .
\end{aligned}
$$

Since $\omega_{i} \omega_{j} / \omega_{1}$ is an $n$th root of -1 reducing to $\omega_{i}$ for $j=1$, it is clear that $\bar{v}_{i}(y, \rho) \equiv \bar{v}_{1}\left(y, \rho \omega_{i} / \omega_{1}\right)$. For $\bar{v}_{1}(y)$ we have the development

$$
\bar{v}_{1}(y, \rho)=\frac{-\omega_{1}}{n \rho^{n-1}} e^{-\rho \omega_{1} v}\left(1+\sum_{l=1}^{l=m} \frac{B_{l}(y)}{\left(\rho \omega_{1}\right)^{l}}+\frac{A_{1}(y, \rho)}{\rho^{m+1}}\right)
$$

where $A_{1}(y, \rho)$ is analytic at infinity in the $\rho$-plane, and the functions $B_{l}(y)$ are continuous together with their derivatives of all orders on $(0,1)$ for $l=1$, $\cdots, m$. Thus

$$
\bar{v}_{i}(y, \rho)=\frac{-\omega_{i}}{n \rho^{n-1}} e^{-\rho \omega_{i} v}\left(1+\sum_{l=1}^{l=m} \frac{B_{l}(y)}{\left(\rho \omega_{i}\right)^{l}}+\frac{A_{i}(y, \rho)}{\rho^{m+1}}\right),
$$

$i=1, \cdots, n$, and

$$
\vartheta_{i}(y, \rho)=\frac{-\omega_{i}}{n \rho^{n-1}} e^{-\rho \omega_{i y}}\left(1+\sum_{l=1}^{l=m} \frac{B_{l}(y)}{\left(\rho \omega_{i}\right)^{l}}+\frac{E_{i}(y, \rho)}{\rho^{m+1}}\right),
$$


$i=1, \cdots, n$, as we were to show. Since the functions $B_{l}(y)$ are expressible in terms of the coefficients $A_{l k}(y)$ which occur in the asymptotic forms for $u_{1}{ }^{(k)}, \cdots, u_{n}{ }^{(k)}$ and which are independent of the sector $S$, they are independent of the sector $S$.

THEOREM XXXII. If $f(x)$ is summable on $(0,1)$; and if $G(x, y ; \lambda)$ is the Green's function associated with the regular differential system of order $n=2 \mu-1$ of the type described above; and if

$$
\begin{aligned}
& F_{1}{ }^{0} k(x, y, \rho) \equiv-\sum_{i=1}^{i=\mu} \sum_{s=0}^{s=k}\left(\rho \omega_{i}\right)^{s} \omega_{i} e^{\rho \omega_{i}(x-y)} \sum_{\alpha+\beta=k-8} A_{\alpha k}(x) B_{\beta}(y), \\
& F_{1}{ }^{1} k(x, y, \rho) \equiv+\sum_{i=\mu+1}^{i=n} \sum_{\delta=0}^{s=k}\left(\rho \omega_{i}\right)^{s} \omega_{i} e^{\rho \omega_{i}(x-y)} \sum_{\alpha+\beta=k=\varepsilon} A_{\alpha k}(x) B_{\beta}(y) \text {, } \\
& F_{2}{ }^{0} k(x, y, \rho) \equiv-\sum_{i=1}^{i=\mu-1} \sum_{s=0}^{s=k}\left(\rho \omega_{i}\right)^{s} \omega_{i} e^{\rho \omega_{i}(x-y)} \sum_{\alpha+\beta=k-s} A_{\alpha k}(x) B_{\beta}(y), \\
& F_{2}{ }^{1} k(x, y, \rho) \equiv+\sum_{i=\mu}^{i=n} \sum_{s=0}^{s=k}\left(\rho \omega_{i}\right)^{s} \omega_{i} e^{\rho \omega_{i}(x-y)} \sum_{\alpha+\beta=k-\varepsilon} A_{\alpha k}(x) B_{\beta}(y),
\end{aligned}
$$

where $A_{\alpha k}(x)$ is the coefficient of $\left(\rho \omega_{i}\right)^{-\alpha}$ in the asymptotic form for $u_{i}^{(k)}(x)$ on $S$, $B_{\beta}(y)$ the coefficient of $\left(\rho \omega_{i}\right)^{-\beta}$ in the form for $v_{i}(y)$, then

$$
\begin{aligned}
& \lim _{R \rightarrow \infty} \int_{0}^{1} f(y) \int_{\gamma_{1}}\left(1-\frac{\rho^{4 n}}{R^{4 n}}\right)^{k+l}\left(n \rho^{n-1}\left\{\frac{\partial^{k} G}{\partial x^{k}} ; \frac{\partial^{k} G}{\partial x^{k}}\right\}-\left\{F_{1{ }^{0}} ; F_{1}{ }^{1} k\right\}\right) d \rho d y=0, \\
& \lim _{R \rightarrow \infty} \int_{0}^{1} f(y) \int_{\gamma_{2}}\left(1-\frac{\rho^{4 n}}{R^{4 n}}\right)^{k+l}\left(n \rho^{n-1}\left\{\frac{\partial^{k} G}{\partial x^{k}} ; \frac{\partial^{k} G}{\partial x^{k}}\right\}-\left\{F_{2}{ }^{0}{ }_{k} ; F_{2}{ }^{1}{ }_{k}\right\}\right) d \rho d y=0,
\end{aligned}
$$

uniformly, $0<a \leqq x \leqq b<1, l \geqq 0$. The expression

$$
\frac{1}{2 \pi i} \frac{\partial^{k}}{\partial x^{k}} \int_{0}^{1} f(y) \int_{C}\left(1-\frac{\lambda^{4}}{\Lambda^{4}}\right)^{k+l} G(x, y ; \lambda) d \lambda d y
$$

is equivalent on any interval $(a, b)$ completely interior to $(0,1)$ to a linear combination with coefficients $A_{\alpha k}(x)$ of means of order $k+l, l \geqq 0$, formed from the Fourier series and their derived series to order $k$ for the functions $f(x) B_{0}(x)$ $\equiv f(x), f(x) B_{1}(x), \cdots, f(x) B_{k}(x)$. On any interval $(a, b)$ the problem of the derived series of Birkhoff of order $n=2 \mu-1$ is reduced to a problem in the theory of derived Fourier series.

Case I. $\rho$ on $S_{1}{ }^{\prime}$. We take $m=k$ in the asymptotic forms of Theorem II' and Lemma XIV. We then compute the asymptotic form of 


$$
n \rho^{n-1}\left\{\frac{\partial^{k}}{\partial x^{k}} G\left(x, y ; \rho^{n}\right) ; \frac{\partial^{k}}{\partial x^{k}} G\left(x, y ; \rho^{n}\right)\right\}
$$

on $S_{\mathbf{1}}{ }^{\prime}$ by methods like those used in Theorem VII. We have

$$
\begin{aligned}
& g(x, y)=\left\{\frac{1}{2} \sum_{i=1}^{i=n} u_{i}(x) v_{i}(y) ;-\frac{1}{2} \sum_{i=1}^{i=n} u_{i}(x) v_{i}(y)\right\},
\end{aligned}
$$

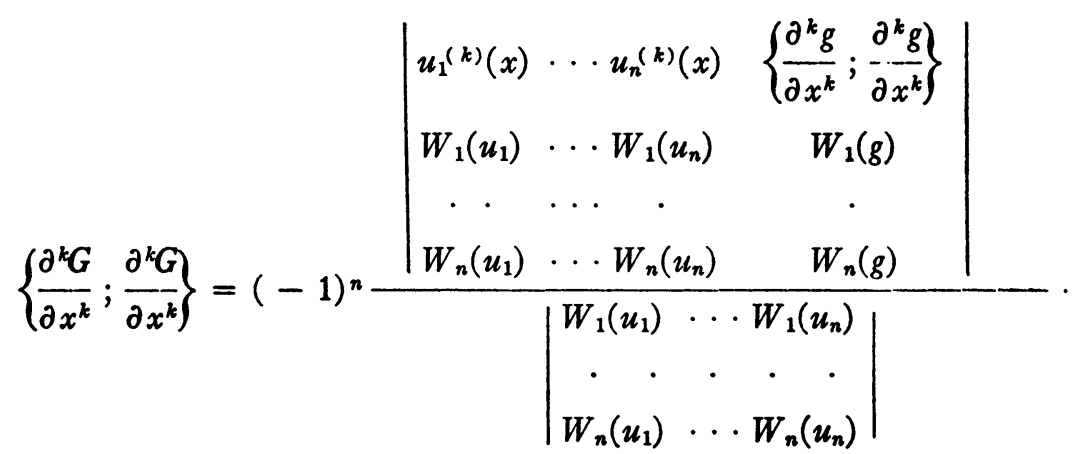

We multiply the first $\mu$ columns in the numerator by $\frac{1}{2} v_{1}(y), \cdots, \frac{1}{2} v_{\mu}(y)$ respectively, the next $\mu-1$ by $-\frac{1}{2} v_{\mu+1}(y), \cdots, \frac{1}{2} v_{n}(y)$, and add to the last. We then take the first term of the new last column outside the determinant. The result of substituting the asymptotic forms for $u_{1}, \cdots, u_{n}, v_{1}, \cdots, v_{n}$ is

$$
\begin{aligned}
n \rho^{n-1}\left\{\frac{\partial^{k} G}{\partial x^{k}} ; \frac{\partial G^{k}}{\partial x^{k}}\right\}= & \left\{F_{1^{0} k}-\sum_{i=1}^{i=\mu} e^{\rho \omega_{i}(x-y)} \frac{m_{i}(x, y, \rho)}{\rho} ;\right. \\
& \left.F_{1}{ }^{1}{ }_{k}+\sum_{i=\mu+1}^{i=n} e^{\rho \omega_{i}(x-y)} \frac{m_{i}(x, y, \rho)}{\rho}\right\}+\frac{\Delta_{1}(k)}{\left[\theta_{0}\right]+e^{\rho \omega_{i}}\left[\theta_{1}\right]}
\end{aligned}
$$

where $\Delta_{1}{ }^{(k)}$ is a determinant which differs from the determinant $\Delta_{1}$ of Theorem VII only in having the elements of the first row changed to

$$
\begin{array}{rlrl}
a_{1 j} & =\left(\rho \omega_{j}\right)^{k} e^{\rho \omega_{j} x}[1], & j=1, \cdots, \mu ; \\
a_{1 j} & =\left(\rho \omega_{j}\right)^{k} e^{\rho \omega_{j}(x-1)}[1], & j=\mu+1, \cdots, n ; \\
a_{1, n+1} & =0 .
\end{array}
$$

Thus we find

$$
\begin{aligned}
& \left(1-\frac{\rho^{4 n}}{R^{4 n}}\right)^{k+l}\left(n \rho^{n-1}\left\{\frac{\partial^{k} G}{\partial x^{k}} ; \frac{\partial^{k} G}{\partial x^{k}}\right\}-\left\{F_{1^{0} k} ; F_{1}{ }^{1} k\right\}\right)=\left\{-\sum_{i=1}^{i=\mu} e^{\rho \omega_{i}(x-y)} \frac{m_{i}}{\rho}\right. \\
& \left.+\sum_{i=\mu+1}^{i=n} e^{\rho \omega_{i}(x-y)} \frac{m_{i}}{\rho}\right\}+\left(1-\frac{\rho^{4 n}}{R^{4 n}}\right)^{k} \rho^{k}\left(\sum_{i=1}^{i=\mu} e^{\rho \omega_{i} x} m_{n+i}+\sum_{i=\mu+1}^{i=n} e^{\rho \omega_{i}(x-1)} m_{n+i}\right)
\end{aligned}
$$


where the functions $m$ are uniformly bounded for all $x$ and $y$ on $(0,1)$ and for all $\rho$ on $S_{1}{ }^{\prime}$. Consequently, by Lemmas III, IV, V,

$$
\int_{\gamma_{1}}\left(1-\frac{\rho^{4 n}}{R^{4 n}}\right)^{k+l}\left(n \rho^{n-1}\left\{\frac{\partial^{k} G}{\partial x^{k}} ; \frac{\partial^{k} G}{\partial x^{k}}\right\}-\left\{F_{1 k}{ }^{k} ; F_{1 k}{ }^{k}\right\}\right) d \rho
$$

is uniformly bounded, $0<a \leqq x \leqq b<1,0 \leqq y \leqq 1$, for all $\rho$ on $S_{1}{ }^{\prime}$. Next, on integrating over $(\alpha, x)$ with respect to $y$ just as we did in Theorem VIII, we find

$$
\begin{aligned}
\int_{\alpha}^{x} \int_{\gamma_{1}} & \left(1-\frac{\rho^{4 n}}{R^{4 n}}\right)^{k+l}\left(n \rho^{n-1}\left\{\frac{\partial^{k} G}{\partial x^{k}} ; \frac{\partial^{k} G}{\partial x^{k}}\right\}-\left\{F_{1^{0} k} ; F_{1}{ }^{1} k\right\}\right) d \rho d y \\
= & \int_{\alpha}^{x} \int_{\gamma_{1}}\left\{-\sum_{i=1}^{i=\mu} e^{\rho \omega_{i}(x-y)} \frac{m_{i}}{\rho} ;+\sum_{i=\mu+1}^{i=n} e^{\rho \omega_{i}(x-y)} \frac{m_{i}}{\rho}\right\} d \rho d y \\
& +\int_{\gamma_{1}}\left(1-\frac{\rho^{4 n}}{R^{4 n}}\right)^{(k-1)+(l+1)} \rho^{k-1}\left(\sum_{i=1}^{i=\mu} e^{\rho \omega_{i} x} m_{n+i}+\sum_{i=\mu+1}^{i=n} e^{\rho \omega_{i}(x-1) m_{n+i}}\right) d \rho .
\end{aligned}
$$

The expression on the right approaches zero uniformly, $0<a \leqq x \leqq b<1$, as $R \rightarrow \infty$ by Lemmas IV, V, VI. Thus

$$
\lim _{R \rightarrow \infty} \int_{\alpha}^{\rho} \int_{\gamma_{1}}\left(1-\frac{\rho^{4 n}}{K^{4 n}}\right)^{k+l}\left(n \rho^{n-1}\left\{\frac{\partial^{k} G}{\partial x^{k}} ; \frac{\partial^{k} G}{\partial x^{k}}\right\}-\left\{F_{1^{i} k} ; F_{1 k}{ }^{1}\right\}\right) d \rho d y=0,
$$

uniformly, $0<a \leqq x \leqq b<1$.

With the aid of Theorem IV the present theorem is established in the case of the first integral.

Case II. $\rho$ on $S_{2}{ }^{\prime}$. We compute the asymptotic form for

$$
n \rho^{n-1}\left\{\frac{\partial^{k} G}{\partial x^{k}} ; \frac{\partial^{k} G}{\partial x^{k}}\right\}
$$

in a manner analogous to that used in Case II of Theorm VII. We then follow our usual line of attack to validate the assertion concerning the second integral of this theorem.

We notice that in the case of Fourier series, as given in the differential system of order $n=2 \mu-1$ analysed in \$III,

$$
\begin{aligned}
& \bar{F}_{1}{ }^{0} \equiv-\sum_{i=1}^{i=\mu}\left(\rho \omega_{i}\right)^{8} \omega_{i} e^{\rho \omega_{i}(x-y)}, \\
& \bar{F}_{1,}^{1} \equiv+\sum_{i=\mu+1}^{i=n}\left(\rho \omega_{i}\right)^{8} \omega_{i} e^{\rho \omega_{i}(x-y)}, \\
& \bar{F}_{2}^{0} \equiv-\sum_{i=1}^{i=\mu-1}\left(\rho \omega_{i}\right)^{8} \omega_{i} e^{\rho \omega_{i}(x-y)}, \\
& \bar{F}_{2 ⿺} \equiv+\sum_{i=\mu}^{i=n}\left(\rho \omega_{i}\right)^{8} \omega_{i} e^{\rho \omega_{i}(x-y)} .
\end{aligned}
$$


Thus

$$
F_{i k} \equiv \sum_{i=0}^{:-k} \bar{F}_{i} ; \sum_{\alpha+\beta=k-\epsilon} A_{\alpha k}(x) B_{\beta}(y), \quad i=0,1, \quad j=1,2 .
$$

Since the functions $A$ and $B$ are independent of the sector $S$, the important relation between the derived series of Birkhoff and the derived series of Fourier, whose complete statement appears above, is now demonstrated.

It is now proposed to investigate a few of the leading properties of the derived series of Fourier series; some of them can be extended to Birkhoff series. The results which we shall present have all been obtained by W. $\mathrm{H}$. Young,* who used methods different from ours.

Theorem XXXIII. If $\phi(x)$ is a k-fold integral in the sense of Lebesgue on $(0,1)$, and if $G(x, y ; \lambda)$ is the Green's function associated with the differential system $u^{\prime}+\lambda u=0, u(0)-u(1)=0$, then

$$
\begin{aligned}
& \lim _{p \rightarrow \infty}\left(\frac{1}{2 \pi i} \int_{0}^{1} \phi^{(k)}(y) \int_{C_{\nu}}\left(1-\frac{\lambda^{4}}{\Lambda_{i}^{4}}\right)^{k+l} G(x, y ; \lambda) d \lambda d y\right. \\
& \left.\quad-\frac{1}{2 \pi i} \frac{\partial^{k}}{\partial x^{k}} \int_{0}^{1} \phi(y) \int_{C_{\nu}}\left(1-\frac{\lambda^{4}}{\Lambda_{y}^{4}}\right)^{k+l} G(x, y ; \lambda) d \lambda d y\right)=0,
\end{aligned}
$$

uniformly, $0<a \leqq x \leqq b<1$, for $l \geqq 0$. In other words, the Cesdro sums of order $k+l, l \geqq 0$, for the kth derived Fourier series of $\phi(x)$ and for the Fourier series of $\phi^{(k)}(x)$ are equivalent on $(a, b)$.

By Theorems IX and XXXII we need consider merely the differences

$$
\begin{aligned}
& \int_{0}^{x} \int_{\gamma_{1}}\left(1-\frac{\lambda^{4}}{\Lambda^{4}}\right)^{k+l}\left(\phi(y) \lambda^{k}(-1)^{k} e^{\lambda(y-x)}-\phi^{(k)}(y) e^{\lambda(y-x)}\right) d \lambda d y \\
& +\int_{1}^{x} \int_{\gamma_{2}}\left(1-\frac{\lambda^{4}}{\Lambda^{4}}\right)^{k+l}\left(\phi(y) \lambda^{k}(-1)^{k} e^{\lambda(y-x)}-\phi^{(k)}(y) e^{\lambda(y-x)}\right) d \lambda d y .
\end{aligned}
$$

By integration by parts we can show

$$
\begin{aligned}
\int_{0}^{x} \phi(y)(-1)^{k} \lambda^{k} e^{\lambda(y-x)} d y= & \sum_{i=1}^{i-k}(-1)^{k-i+1} \lambda^{k-i}\left(\phi^{(i-1)}(x)-\phi^{(i-1)}(0) e^{-\lambda x}\right) \\
& +\int_{0}^{x} \phi^{(k)}(y) e^{\lambda(y-x)} d y,
\end{aligned}
$$

W. H. Young, Proceedings of the London Mathematical Society, ser. 2, vol. 17 (1918), pp. 195-236. 


$$
\begin{aligned}
\int_{1}^{x} \phi(y)(-1)^{k} \lambda^{k} e^{\lambda(y-x)} d y= & \sum_{i=1}^{i=k}(-1)^{k-i+1} \lambda^{(k-i)}\left(\phi^{(i-1)}(x)-\phi^{(i-1)}(1) e^{\lambda(1-x)}\right) \\
& +\int_{1}^{x} \phi^{(k)}(y) e^{\lambda(y-x)} d y .
\end{aligned}
$$

On substituting these expressions we find

$$
\begin{aligned}
\sum_{i=1}^{i=k}( & -1)^{k-i+1} \phi^{(i-1)}(x) \int_{C} \lambda^{k-i}\left(1-\frac{\lambda^{4}}{\Lambda^{4}}\right)^{k+l} d \lambda \\
& +\sum_{i=1}^{i=k}(-1)^{k-i} \phi^{(i-1)}(0) \int_{\gamma_{1}} \lambda^{k-i}\left(1-\frac{\lambda^{4}}{\Lambda^{4}}\right)^{(k-i)+(l+i)} e^{-\lambda x} d \lambda \\
& +\sum_{i=1}^{i=k}(-1)^{k-i} \phi^{(i-1)}(1) \int_{\gamma_{2}} \lambda^{k-i}\left(1-\frac{\lambda^{4}}{\Lambda^{4}}\right)^{(k-i)+(l+i)} e^{\lambda(1-x)} d \lambda
\end{aligned}
$$

The integrals over $C$ vanish identically by Cauchy's theorem. The integrals in the remaining terms approach zero uniformly, $0<a \leqq x \leqq b<1$ as $\Lambda \rightarrow \infty$. $l \geqq 0$, by the application of Lemma $\mathrm{V}$.

By Theorem VI, Corollary I, the type of mean employed in summing the two series may be replaced by a Cesàro sum of the same order, $k+l$.

CoROllary I. The $(k+l)$ th Cesàro sum of the kth derived Fourier series for $\phi(x)$ converges almost everywhere to $\phi^{(k)}(x)$; and converges uniformly on $(a, b)$ if $\phi^{(k)}(x)$ is continuous.

Corollary II. We have, if $\psi(x)$ is bounded and summable on $(0,1)$,

$$
\begin{gathered}
\lim _{\nu \rightarrow \infty} \int_{x_{1}}^{x_{2}} \psi(\xi) \frac{1}{2 \pi i} \frac{\partial^{k}}{\partial \xi^{k}} \int_{0}^{1} \phi(y) \int_{C_{\nu}}\left(1-\frac{\lambda^{4}}{\Lambda_{\nu}^{4}}\right)^{k+l} G(\xi, y ; \lambda) d \lambda d y d \xi \\
=\int_{x_{1}}^{x_{2}} \psi(\xi) \phi^{(k)}(\xi) d \xi
\end{gathered}
$$

for all $x_{1}$ and $x_{2}$ interior to $(0,1)$.

On applying the preceding theorem and its two corollaries to Theorem XXXII we find

THEOREM XXXIV. If $\phi(x)$ is a k-fold integral in the sense of Lebesgue, $0 \leqq x \leqq 1$, then

$$
\lim _{l \rightarrow \infty} \frac{1}{2 \pi i} \frac{\partial^{k}}{\partial x^{k}} \int_{0}^{1} \phi(y) \int_{C_{\nu}}\left(1-\frac{\lambda^{4}}{\Lambda_{\nu}^{4}}\right)^{k+l} G(x, y ; \lambda) d \lambda d y=\phi^{(k)}(x)
$$

almost everywhere, $0<x<1$; and if $\phi^{(k)}(x)$ is continuous, the convergence to the limit is uniform, $0<a \leqq x \leqq b<1$. 
By Corollary I of Theorem XXXIII and by the last part of Theorem XXXII, we see that

$$
\lim _{\nu \rightarrow \infty} \frac{1}{2 \pi i} \frac{\partial^{k}}{\partial x^{k}} \int_{0}^{1} \phi(y) \int_{C_{\nu}}\left(1-\frac{\lambda^{4}}{\Lambda_{\nu}^{4}}\right)^{k+l} G(x, y ; \lambda) d \lambda d y=\Phi_{k}(x)
$$

almost everywhere, $0<x<1$, the convergence being uniform on $(a, b)$ if $\phi^{(k)}(x)$ is continuous. By Corollary II of 'Theorem XXXIII we can compute the integral of $\Phi_{k}(x)$ by integrating under the limit sign on the left. We have

$$
\Phi_{k-1}(x)-\Phi_{k-1}\left(x_{1}\right)=\int_{x_{1}}^{x} \Phi_{k}(\xi) d \xi, \Phi_{k}(x)=\Phi_{k-1}^{\prime}(x)=\cdots=\Phi_{0}^{(k)}(x) .
$$

Since $\Phi_{0}(x) \equiv \phi(x)$, it follows that $\Phi_{k}(x)=\phi^{(k-1)}(x)$ and the theorem is proved.

THEOREM XXXV. If $f(x)$ is summable on $(0,1)$, then the convergence at a point $x_{0}$ interior to $(0,1)$, of the $(k+l)$ th Cesaro mean, $l \geqq 0$, of the kth derived series of the Fourier series for $f(x)$ is independent of the nature of the function outside an arbitrarily small neighborhood of the point $x_{0}{ }^{*}$

We consider the differential system $u^{\prime}+\lambda u=0, u(0)-u(1)=0$, starting from Theorem XXXII just as we did from Theorem XIX in proving Theorem XIV. The study of the $(k+l)$ th mean of the $k$ th derived Fourier series reduces to that of the expression

$$
\begin{aligned}
& \frac{1}{2 \pi} \int_{0}^{x_{0}} f\left(-z+x_{0}\right) \int_{-1}^{+1}\left(1-\phi^{4}\right)^{k+l}(-i)^{k} \phi^{k} \Lambda^{k+1} e^{-\Lambda i \phi z} d \phi d z \\
& \quad+\frac{1}{2 \pi} \int_{0}^{1-x_{0}} f\left(z+x_{0}\right) \int_{-1}^{+1}\left(1-\phi^{4}\right)^{k+l}(-i) \phi^{k} \Lambda^{k+1} e^{\Delta i \phi z} d \phi d z
\end{aligned}
$$

where the integrals with respect to $\phi$ are taken over the real axis. We let $A$ be an arbitrarily small fixed positive quantity, which we may suppose less than $x_{0}$ and $1-x_{0}$; we denote by $h$ the function

$$
\frac{d^{k+1}}{d \phi^{k+1}}\left[\left(1-\phi^{4}\right)^{k+l} \phi^{k}\right]
$$

which is summable on $(-1,1)$ for $l \geqq 0$. Then on integrating by parts $k+1$ times we find

$$
\int_{-1}^{+1}\left(1-\phi^{4}\right)^{k+l} \phi^{k} \Lambda^{k+1} e^{ \pm \Lambda i \phi z} d \phi=\frac{(\mp 1)^{k+1}}{z^{k+1}} \int_{-1}^{+1} h e^{ \pm \Delta i \phi z} d \phi,
$$

-W. H. Young, Proceedings of the London Mathematical Society, ser. 2, vol. 17 (1918) pp. 195-236. 
an expression which is uniformly bounded for $z \geqq A, l \geqq 0$, and which approaches zero with $1 / \Lambda$ for each positive value of $z$, by the theorem of Riemann-Lebesgue. It follows that in the expression above the integrals over $\left(A, x_{0}\right)$ and $\left(A, 1-x_{0}\right)$ have the limit zero as $\Lambda \rightarrow \infty$. Therefore the convergence of the $(k+l)$ th mean, $l \geqq 0$, at $x=x_{0}$ depends on the behavior of the sum

$$
\begin{aligned}
& \frac{1}{2 \pi} \int_{0}^{\Lambda} f\left(-z+x_{0}\right) \int_{-1}^{+1}\left(1-\phi^{4}\right)^{k+l}(-i)^{k} \phi^{k} \Lambda^{k+1} e^{-\Lambda i \phi z} d \phi d z \\
& +\frac{1}{2 \pi} \int_{0}^{\Lambda} f\left(z+x_{0}\right) \int_{-1}^{+1}\left(1-\phi^{4}\right)^{k+l}(-i)^{k} \phi^{k} \Lambda^{k+1} e^{\Lambda i \phi z} d \phi d z .
\end{aligned}
$$

This establishes the theorem.

An immediate consequence of this result is

TheOREM XXXVI. If $f(x)$ is summable on $(0,1)$, the behavior of the expression

$$
\frac{1}{2 \pi i} \frac{\partial^{k}}{\partial x^{k}} \int_{0}^{1} f(y) \int_{C_{y}}\left(1-\frac{\lambda^{4}}{\Lambda_{y}^{4}}\right)^{k+l} G(x, y ; \lambda) d \lambda d y, \quad l \geqq 0,
$$

at the point $x=x_{0}, 0<x_{0}<1$, as $\nu \rightarrow \infty$ is independent of the nature of $f(x)$ outside an arbitrarily small neighborhood of $x_{0}$.

We now proceed to two other theorems of more general character, with a view to showing the effectiveness of the method here considered in discussing the derived series of Fourier series.

TheOREM XXXVII. If $f(x)$ is summable on $(0,1)$; and if

$$
F(x)=\frac{f(x)-f\left(2 x_{0}-x\right)}{2\left(x-x_{0}\right)}
$$

is summable on $(0,1)$ when $x_{0}$ is interior to $(0,1)$ and $f(x)$ is defined for all values of $x$ by the identity $f(x) \equiv f(x+1)$; and if $S_{\Lambda}^{(D)}, \Sigma_{\Lambda}^{(D)}$ denote the $[\Lambda]$ th Cesdro sums of order $l$ for a Fourier series and its first derived series respectively, then

$$
\lim _{\Delta \rightarrow \infty}\left(\Sigma_{\Delta}^{(l+1)}(f)-5 S_{\Lambda}^{(l+1)}(F)+4 S_{\Delta}^{(l)}(F)\right)=0, \quad x=x_{0}, \quad l \geqq 0 .
$$

In particular, if $f(x)$ is continuous and $\lim _{x \rightarrow x_{0}} F(x)=A$, then

$$
\lim _{\Delta \rightarrow \infty} \Sigma_{\Delta}^{(l+1)}(f)=A, \quad x=x_{0}, \quad l>0 . *
$$

-W. H. Young, Proceedings of the London Mathematical Society, ser. 2, vol. 17 (1918) pp. 195-236. 
It is sufficient to demonstrate that

$$
\lim _{\Delta \rightarrow \infty}\left(\Sigma_{\Delta}^{(1)}(f)-5 S_{\Delta}^{(1)}(F)+4 S_{\Delta}^{(0)}(F)\right)=0, \quad x=x_{0} .
$$

By taking $k=1, l=0$, in the final expression of Theorem XXXV and noticing that

$$
\int_{-1}^{+1}\left(1-\phi^{4}\right) \phi \cos \Lambda \phi z d \phi=0
$$

we find that we can replace $\Sigma_{\Lambda}^{(1)}(f)$ by the expression

$$
\frac{1}{2 \pi} \int_{0}^{\Lambda}\left(f\left(z+x_{0}\right)-f\left(-z+x_{0}\right)\right) \int_{-1}^{+1}\left(1-\phi^{4}\right) \phi \Lambda^{2} \sin \Lambda \phi z d \phi d z,
$$

in the sense that this expression has the same behavior as $\Lambda \rightarrow \infty$. On integrating by parts we find

$$
\int_{-1}^{+1}\left(1-\phi^{4}\right) \phi \Lambda^{2} \sin \Lambda \phi z d \phi=\frac{1}{z} \int_{-1}^{+1}\left(1-5 \phi^{4}\right) \Lambda \cos \Lambda \phi z d \phi .
$$

Hence we can replace $\Sigma_{\Lambda}^{(1)}(f)$ by

$$
\frac{1}{2 \pi} \int_{0}^{\Lambda} \frac{f\left(z+x_{0}\right)-f\left(-z+x_{0}\right)}{z} \int_{-1}^{+1}\left(1-5 \phi^{4}\right) \Lambda \cos \Lambda \phi z d \phi d z .
$$

We note that $F\left(-z+x_{0}\right)+F\left(z+x_{0}\right)=\left[f\left(z+x_{0}\right)-f\left(-z+x_{0}\right)\right] / z$. Thus we can replace $S_{\Lambda}^{(1)}(F)$ and $S_{\Lambda}^{(0)}(F)$ by

$$
\frac{1}{2 \pi} \int_{0}^{\Lambda} \frac{f\left(z+x_{0}\right)-f\left(-z+x_{0}\right)}{z} \int_{-1}^{+1}\left(1-\phi^{4}\right) \Lambda \cos \Lambda \phi z d \phi d z
$$

and

$$
\frac{1}{2 \pi} \int_{0}^{\Lambda} \frac{f\left(z+x_{0}\right)-f\left(-z+x_{0}\right)}{z} \int_{-1}^{+1} \Lambda \cos \Lambda \phi z d \phi d z
$$

respectively. When we make the three replacements in the expression

$$
\Sigma_{\Delta}^{(1)}(f)-5 S_{\Lambda}^{(1)}(F)+4 S_{\Delta}^{(0)}(F)
$$

we find that it then reduces to zero; that is to say, for $x=x_{0}$,

$$
\lim _{\Delta \rightarrow \infty}\left(\Sigma_{\Delta}^{(1)}(f)-5 S_{\Delta}^{(1)}(F)+4 S_{\Delta}^{(0)}(F)\right)=0 .
$$

The remainder of the theorem follows at once. 
TheORem XXXVIII. The first derived series of the Fourier series for a function of bounded variation, $\chi(x)$, is summable $(C, l), l>0$, to $\chi^{\prime}(x)$ almost everywhere on $(0,1) .^{*}$

We employ here the concept of integration with respect to a function of bounded variation. We let $x_{0}$ be a point interior to $(0,1)$ for which the function

$$
X(z)=\frac{\chi\left(z+x_{0}\right)-\chi\left(-z+x_{0}\right)}{2}-z \chi^{\prime}\left(x_{0}\right)
$$

is defined and satisfies the conditions

$$
X(+0)=0, \quad \lim _{z \rightarrow 0} \frac{1}{z} \int_{0}^{z}|d X|=0 .
$$

Young has shown that the set of points thus defined is of measure 1.

Taking $G(x, y ; \lambda)$ as the Green's function for the system $u^{\prime}+\lambda u=0$, $u(0)-u(1)=0$, we find for $x=x_{0}$

$$
\begin{gathered}
\frac{\partial}{\partial x} \int_{0}^{1} x(y) \int_{c_{\nu}}\left(1-\frac{\lambda^{4}}{\Lambda_{v}^{4}}\right)^{l} G(x, y ; \lambda) d \lambda d y \\
=\int_{0}^{x_{0}} x(y) \int_{\gamma_{1}}\left(1-\frac{\lambda^{4}}{\Lambda_{v}^{4}}\right)^{l} \lambda e^{\lambda\left(y-x_{0}\right)} d \lambda d y \\
\quad+\int_{x_{0}}^{1} x(y) \int_{\gamma_{2}}\left(1-\frac{\lambda^{4}}{\Lambda_{\nu}^{4}}\right)^{l} \lambda e^{\lambda\left(y-x_{0}\right)} d \lambda d y \\
+\int_{0}^{1} x(y) \int_{\gamma_{1}}\left(1-\frac{\lambda^{4}}{\Lambda_{v}^{4}}\right)^{l} \frac{-\lambda e^{-\lambda x_{0} e^{\lambda(y-1)}}}{1-e^{-\lambda}} d \lambda d y \\
+\int_{0}^{1} x(y) \int_{\gamma_{2}}\left(1-\frac{\lambda^{4}}{\Lambda_{v}^{4}}\right)^{l} \frac{\lambda e^{\lambda\left(1-x_{0}\right)} e^{\lambda y}}{e^{\lambda}-1} d \lambda d y .
\end{gathered}
$$

In the third integral we have

$$
\begin{aligned}
\left|\int_{0}^{1} \chi(y) \lambda e^{\lambda(y-1)} d y\right| & =\left|\chi(1)-\chi(0) e^{-\lambda}-\int_{0}^{1} e^{\lambda(y-1)} d \chi\right| \\
& \leqq|\chi(1)|+|\chi(0)|+\int_{0}^{1}|d \chi| \leqq M,
\end{aligned}
$$

-W. H. Young, Proceedings of the London Mathematical Society, ser. 2, vol. 13 (1914) pp. 13-28. 
so that for $l>0$ it approaches zero with $1 / \Lambda$ in accord with Lemma V. By a similar argument we can dispose of the fourth integral. The two remaining integrals can be changed into the form

$$
\begin{aligned}
& \frac{1}{2 \pi} \int_{0}^{x_{0}} \chi\left(-z+x_{0}\right) \int_{-1}^{+1}\left(1-\phi^{4}\right)^{l}(-i \phi) \Lambda^{2} e^{-\Delta^{i} \phi z} d \phi d z \\
& \quad+\frac{1}{2 \pi} \int_{0}^{1-x_{0}} \chi\left(z+x_{0}\right) \int_{-1}^{+1}\left(1-\phi^{4}\right)^{l}(-i \phi) \Lambda^{2} e^{\Delta i \phi z} d \phi d z
\end{aligned}
$$

as was done in Theorems XIV and XXXV. We take $A$ positive but less than $x_{0}$ and $1-x_{0}$. Then we show the integrals over $\left(A, x_{0}\right)$ and $\left(A, 1-x_{0}\right)$ negligible, in the following manner: we integrate by parts with respect to $z$ in each of them and apply the inequalities of Lemma VII to the resulting expressions. The discussion has many points of similarity with the first part of that given in Theorem XIV. Thus we consider

$$
\frac{1}{\pi} \int_{0}^{\Lambda}\left(X(z)+z \chi^{\prime}\left(x_{0}\right)\right) \int_{-1}^{+1}\left(1-\phi^{4}\right) \phi \Lambda^{2} \sin \Lambda \phi z d \phi d z, \quad l>0 .
$$

It is readily shown that

$$
\lim _{\Delta \rightarrow \infty} \frac{1}{\pi} \int_{0}^{\Lambda} z \int_{-1}^{+1}\left(1-\phi^{4}\right)^{l} \phi \Lambda^{2} \sin \Lambda \phi z d \phi d z=1
$$

To prove the present theorem, then, it remains to show that

$$
\lim _{\Lambda \rightarrow \infty} \int_{0}^{\Lambda} X(z) \int_{-1}^{+1}\left(1-\phi^{4}\right)^{l} \phi \Lambda^{2} \sin \Lambda \phi z d \phi d z=0 .
$$

This is easily accomplished after an integration by parts with the aid of the inequalities of Lemma VII; the method is that used by Hardy for the proof of the last step in Theorem XIV and by Young for the present theorem. This finishes the proof.

We leave the reader to apply the last two theorems to the study of the derived series of Birkhoff of order $n=2 \mu-1$.

\section{THE SERIES FOR $n=2 \mu$}

The regular differential system

$$
\begin{aligned}
& u^{(n)}+\star+p_{2} u^{(n-2)}+\cdots+\left(p_{n}+\lambda\right) u=0, \\
& W_{1}(u)=0, \cdots, W_{n}(u)=0, \quad n=2 \mu,
\end{aligned}
$$

where

$$
\begin{aligned}
& W_{i}(u) \equiv \alpha_{i} u^{\left(k_{i}\right)}(0)+\beta_{i} u^{\left(k_{i}\right)}(1)+\cdots, \\
& n-1 \geqq k_{1} \geqq k_{2} \geqq \cdots \geqq k_{n}, \quad k_{i+2}>k_{i},
\end{aligned}
$$


presents certain salient differences from the case $n=2 \mu-1$; but these are differences of form which do not necessitate any essential alteration in the spirit of the method used.

We shall write $\lambda=\rho^{n}$, considering instead of the whole $\lambda$-plane two adjacent sectors $S: R\left(\rho \omega_{1}\right) \leqq R\left(\rho \omega_{2}\right) \leqq \cdots \leqq R\left(\rho \omega_{n}\right)$ in the $\rho$-plane. This region we shall call $\Sigma$. The sector $S$ is a region $T$ of $\S I$ for $c=0$. On employing the asymptotic forms for solutions $u_{1}, \cdots, u_{n}$ on a region $T$ including $S$ in its interior we ascertain the distribution of the characteristic values in $S .^{*}$ On one of the bounding rays of a sector $S, R\left(\rho \omega_{\mu}\right)=R\left(\rho \omega_{\mu+1}\right)=0$; we arrange the two adjacent sectors $S$ forming $\Sigma$ so that they have such a bounding ray in common. Then the characteristic values of $\rho$ on $\Sigma$ are distributed in two series, asymptotically near points equally spaced at distance $2 \pi$ along each of two lines parallel to the bisecting ray of $\Sigma$; these are simple characteristic values except in the case of a value common to the two series, which is always double.

We now prepare the regions $\Sigma^{\prime}, S^{\prime}$ in a manner entirely analogous to that employed in the case $n=2 \mu-1$. The arcs $\Gamma, \gamma$ and the circles $C$ can then be defined. The notations $\sigma, R$ will be carried over from our preceding work.

On any region $S$ we have

$$
\begin{aligned}
& R\left(\rho \omega_{1}\right) \leqq R\left(\rho \omega_{2}\right) \leqq \cdots \leqq R\left(\rho \omega_{\mu-1}\right) \leqq-\beta<0 ; \\
& R\left(\rho \omega_{\mu}\right)=R\left(\rho \omega_{\mu+1}\right)=0, \text { along one bounding ray; } \\
& \omega_{\mu}=-\omega_{\mu+1} ; \\
& R\left(\rho \omega_{n}\right) \geqq R\left(\rho \omega_{n-1}\right) \geqq \cdots \geqq R\left(\rho \omega_{n-2}\right) \geqq \beta>0 .
\end{aligned}
$$

Hence we can consider the whole sector $S^{\prime}$ at once; but we have two roots of -1 , namely $\omega_{\mu}, \omega_{\mu+1}$, which have to receive special attention. This is the reverse of the situation in the case $n=2 \mu-1$, where the sector $S^{\prime}$ had to be divided and where there was on each sector $S_{1}{ }^{\prime}, S_{2}{ }^{\prime}$ a single root of -1 which required separate consideration.

In the present discussion we have to replace Lemmas IV, V, VI. We have

LEMMA IV'. If $m(x, \rho)$ is a function of $x$ and $\rho$ bounded for $0<a \leqq x \leqq b<1$ and for all $\rho$ on $S^{\prime}$, then

$$
\lim _{R \rightarrow \infty} \int_{\gamma} \rho^{k} e^{\rho \omega_{i} x} m d \rho=0 \quad(i=1, \cdots, \mu-1),
$$

- Birkhoff, Rendiconti del Circolo Matematico di Palermo, vol. 36 (1913), p. 117.

$\dagger$ Birkhoff, these Transactions, vol. 9 (1908), p. 386. 


$$
\lim _{R \rightarrow \infty} \int_{\gamma} \rho^{k} e^{\rho \omega_{i}(x-1)} m d \rho=0 \quad(i=\mu+2, \cdots, n),
$$

for any $k$, uniformly, $0<a \leqq x \leqq b<1$.

LEMMA $V^{\prime}$. If $m(x, y, \rho)$ is a function of $x, y$, and $\rho$ bounded for $0<a \leqq x$ $\leqq b<1,0 \leqq y \leqq 1$, and for all $\rho$ on $S^{\prime}$, then

$$
\begin{aligned}
& \int_{\gamma} \rho^{k}\left(1-\frac{\rho^{4 n}}{R^{4 n}}\right) e^{\rho \omega_{\mu} x} m d \rho, \\
& \int_{\gamma} \rho^{k}\left(1-\frac{\rho^{4 n}}{R^{4 n}}\right)^{k+l} e^{\rho \omega_{\mu+1}(x-1)} m d \rho,
\end{aligned}
$$

for any $k \geqq 0$, (1) are uniformly bounded, $0<a \leqq x \leqq b<1,0 \leqq y \leqq 1$, for all $\gamma$ on $S^{\prime}$ when $l=0$, and (2) approach zero uniformly with $1 / R$ for $0<a \leqq x \leqq b$ $<1,0 \leqq y \leqq 1$, when $l>0$.

LEMMA VI'. If $m(x, y, \rho)$ is uniformly bounded, $0 \leqq x \leqq 1,0 \leqq y \leqq 1$, for all $\rho$ on $S^{\prime}$, then

$$
\lim _{R \rightarrow \infty} \int_{\alpha}^{x} \int_{\gamma} e^{\rho \omega_{i}(x-y)} \frac{m}{\rho} d \rho d y=0 \quad(i=1, \cdots, \mu),
$$

uniformly, $0 \leqq \alpha \leqq x \leqq 1 ;$ and

$$
\lim _{R \rightarrow \infty} \int_{\alpha}^{x} \int_{\gamma} e^{\rho \omega_{i}(x-y)} \frac{m}{\rho} d \rho d y=0 \quad(i=\mu+1, \cdots, n),
$$

unıformly, $0 \leqq x \leqq \alpha \leqq 1$.

The proofs are entirely analogous to those used for the preceding lemmas. Lemma III does not need to be replaced.

The Green's function for a system of order $n=2 \mu$ is given by the explicit formula written down in Theorem VII. We proceed to put this expression in a more useful form by multiplying the first $\mu$ columns in the numerator by $\frac{1}{2} v_{1}(y), \cdots, \frac{1}{2} v_{\mu}(y)$ respectively, the next $\mu$ by $-\frac{1}{2} v_{\mu+1}(y), \cdots,-\frac{1}{2} v_{n}(y)$ respectively, and adding to the last. We then take the first term in the new last columu outside the determinant. The result of substituting the asymptotic forms of $u_{1}, \cdots, u_{n}, v_{1}, \cdots, v_{n}$ is then*

$$
\begin{aligned}
n \rho^{n-1} G=\left\{-\sum_{i=1}^{i=\mu} e^{\rho \omega_{i}(x-y)}\left[\omega_{i}\right] ;\right. & \left.+\sum_{i=\mu+1}^{i=n} e^{\rho \omega_{i}(x-y)}\left[\omega_{i}\right]\right\} \\
& +\frac{\Delta_{2}}{\left[\theta_{1}\right] e^{2 \rho \omega_{\mu}}+\left[\theta_{0}\right] e^{\rho \omega_{\mu}}+\left[\theta_{2}\right]}
\end{aligned}
$$

* Tamarkin, Rendiconti del Circolo Matematico di Palermo, vol. 34 (1912), p. 364. 
where $\Delta_{3}$ is a determinant of order $n+1$ in which the element $a_{h i}, h=0,1$, $\cdots, n, j=1,2, \cdots, n+1$ may described as follows :

$a_{0, j}=e^{\rho \omega j x}[1], j=1, \cdots, \mu ; a_{0, j}=e^{\rho \omega j(x-1)}[1], j=\mu+1, \cdots, n ; a_{0, n+1}=0$;

and for $h=1,2, \cdots, n$,

$$
\begin{aligned}
a_{h j} & =\left[\alpha_{h} \omega_{j}^{k_{h}}\right], j=1, \cdots, \mu-1 ; a_{h, \mu}=\left[\alpha_{h} \omega_{\mu}{ }^{k_{h}}\right]+e^{\rho \omega_{\mu}}\left[\beta_{h} \omega_{\mu}{ }^{k_{h}}\right] ; \\
a_{h, \mu+1} & =\left[\alpha_{h} \omega_{\mu+1}^{k_{h}}\right] e^{-\rho \omega_{\mu+1}}+\left[\beta_{h} \omega_{\mu+1}^{k_{h}}\right] ; a_{h j}=\left[\beta_{h} \omega_{i}^{k_{h}}\right], j=\mu+2, \cdots, n ; \\
a_{h, n+1} & =-\sum_{i=1}^{i=\mu} e^{\rho \omega_{i}(1-\nu)}\left[\beta_{h} \omega_{i}{ }^{k_{h}+1}\right]+\sum_{i=\mu+1}^{i=n} e^{-\rho \omega_{i} \nu}\left[\alpha_{h} \omega_{i}^{k_{h}+1}\right] .
\end{aligned}
$$

On $S^{\prime}, 1 /\left\{\left[\theta_{1}\right] e^{2 \rho \omega_{\mu}}+\left[\theta_{0}\right] e^{\rho \omega \mu}+\left[\theta_{2}\right]\right\}$ is bounded.* In short, we are prepared to proceed as we did in the case $n=2 \mu-1$.

We can thus establish Theorems $\mathrm{IX}^{\prime}, \mathrm{XII}^{\prime}, \mathrm{XIII}^{\prime}$, whose statements are identical with those of Theorems IX, XII, XIII, respectively, except for the change from $n=2 \mu-1$ to $n=2 \mu$. In the proof of Theorem $\mathrm{XII}^{\prime}$, essentially the same as that of Theorem XII, we must remark a slight difference. If we let $[\sigma]$ be that part of $\sigma$ on $S^{\prime}$, then

$$
\int_{0}^{1} \int_{[\sigma]} f(y) n \rho^{n-1} G\left(x, y ; \rho^{n}\right) d \rho d y
$$

approaches zero uniformly, $0 \leqq x \leqq 1$, as the center of $\sigma$ recedes indefinitely from the origin. Hence on $\Sigma$,

$$
\int_{0}^{1} \int_{\sigma} f(y) n \rho^{n-1} G\left(x, y ; \rho^{n}\right) d \rho d y
$$

approaches zero in similar fashion. Because of the distribution of the characteristic values when $n=2 \mu$ the circles $\sigma$ remote from the origin may be intersected by the bisecting ray of $\Sigma$, and it is necessary to introduce the corresponding modification of the proof. Theorem XIII', which asserts the equivalence of Birkhoff series of order $n=2 \mu$ and Fourier series on any interval $(a, b)$ completely interior to $(0,1)$, is the strongest theorem possible, in general, as we see by reference to the sine, cosine, and Fourier series in the case $n=2$.

We next introduce

LEMMA VIII'. On any region $S^{\prime}$

$$
\frac{\left[\theta_{1}\right] e^{2 \rho \omega_{\mu}}+\left[\theta_{0}\right] e^{\rho \omega_{\mu}}+\left[\theta_{2}\right]}{\left[\bar{\theta}_{1}\right] e^{2 \rho \omega \mu}+\left[\bar{\theta}_{0}\right] e^{\rho \omega_{\mu}}+\left[\bar{\theta}_{2}\right]}=[1]
$$

if $\theta_{0}=\bar{\theta}_{0}, \theta_{1}=\bar{\theta}_{1}, \theta_{2}=\bar{\theta}_{2}$.

- Birkhoff, Rendiconti del Circolo Matematico di Palermo, vol. 36 (1913), p. 120. 
LEMMA IX'. The integrals

$$
\begin{array}{lr}
\int_{\gamma} \int_{\alpha}^{x} e^{\rho_{i}(1-y)}(m / \rho) d \rho d y & (i=1, \cdots, \mu), \\
\int_{\gamma} \int_{\alpha}^{x} e^{-\rho \omega_{i} y}(m / \rho) d \rho d y & (i=\mu+1, \cdots, n),
\end{array}
$$

where $m(x, y, \rho)$ is bounded for all $x$ and $y$ on $(0,1)$ and for all $\rho$ on $S^{\prime}$, converge uniformly to zero with $1 / R, 0 \leqq x \leqq 1,0 \leqq \alpha \leqq 1$.

We can now obtain Theorems XVII' and XVIII' which may be stated merely by replacing $n=2 \mu-1$ by $n=2 \mu$ in Theorems XVII and XVIII. It is to be noticed that Theorem XVIII' includes as special cases the leading results of Haar and of Walsh cited in the introduction.

We next introduce the determinant $D(x, y, \rho)$ obtained from $\Delta_{\mathbf{s}}$ by replacing each asymptotic expression $[A]$ by its dominating constant term $A$, and deleting the terms involving $e^{\rho \omega_{\mu}}, e^{\rho \omega_{\mu+1}}$. From $\mathcal{D}(0, y, \rho)$ and $\mathscr{D}(1, y, \rho)$ we obtain new determinants $D^{0}(y, \rho), D^{1}(y, \rho)$ by replacing by zero the terms of the first rows explicitly involving the variable $\rho$. We then obtain two theorems similar to Theorems XIX and XX.

Theorem XIX'. If $f(x)$ is summable on $(0,1)$, then

$$
\begin{gathered}
\lim _{R \rightarrow \infty} \int_{0}^{1} f(y) \int_{\gamma}\left(n \rho^{n-1} G\left(x, y ; \rho^{n}\right)-\left\{-\sum_{i=1}^{i-\mu} e^{\rho \omega_{i}(x-y)} \omega_{i} ;+\sum_{i=\mu+1}^{i-n} e^{\rho \omega_{i}(x-y)} \omega_{i}\right\}\right. \\
\left.-\frac{1}{\theta_{2}} \mathcal{D}(x, y, \rho)\right) d \rho d y=0
\end{gathered}
$$

uniformly, $0 \leqq x \leqq 1$.

TheOREM XX'. If $f(x)$ is summabie on $(0,1)$ then

$$
\begin{aligned}
& \lim _{R \rightarrow \infty} \int_{0}^{1} f(y) \int_{\gamma}\left(n \rho^{n-1} G\left(0, y ; \rho^{n}\right)-\sum_{i=\mu+1}^{i=n} \omega_{i} e^{-\rho \omega_{i} \nu} \frac{1}{\theta_{2}} D^{0}(y, \rho)\right) d \rho d y=0, \\
& \lim _{R \rightarrow \infty} \int_{0}^{1} f(y) \int_{\gamma}\left(n \rho^{n-1} G\left(1, y ; \rho^{n}\right)+\sum_{i=1}^{i=\mu} \omega_{i} e^{\rho \omega_{i}(1-y)} \frac{1}{\theta_{2}} D^{1}(y, \rho)\right) d \rho d y=0 .
\end{aligned}
$$

We can next use Lemmas X and XII much as we did in §IV. In fact, we have, with the notations of Lemma $\mathrm{X}$ and Theorem XXI,

$$
\int_{0}^{1} f(y) \int_{\gamma} \omega_{k} e^{\rho \omega_{k}(1-y)} d \rho d y=\int_{0}^{1} f(y) \frac{e^{-R \lambda_{1} y}-e^{-R \lambda_{2} y}}{y} d y
$$


where $\theta_{2}-\theta_{1}=-\pi / n$, for $k=1, \cdots, n$; and

$$
\int_{0}^{1} f(y) \int_{\gamma} \omega_{k} e^{-\rho \omega_{k} y} d \rho d y=\int_{0}^{1} f(y) \frac{e^{-R \lambda_{1} y}-e^{-R \lambda_{2} y}}{y} d y
$$

where $\theta_{2}-\theta_{1}=\pi / n$, for $k=\mu+1, \cdots, n$. Hence we obtain Theorem XXI', whose statement parallels that of Theorem XXI. Likewise there results

Theorem XXII'. If $\phi(x)$ is summable on $(0,1)$, and if

$$
\Phi_{1}(x)=\frac{1}{x} \int_{0}^{x} \phi(x) d x, \quad \Phi_{2}(x)=\frac{1}{x} \int_{0}^{x} \phi(1-x) d x
$$

are of bounded variation near $x=0$, then

$$
\lim _{R \rightarrow \infty} \frac{1}{2 \pi i} \int_{0}^{1} \phi(y) \int_{\gamma} n \rho^{n-1} G\left(k, y ; \rho^{n}\right) d \rho d y=A_{k} \Phi_{1}(+0)+B_{k} \Phi_{2}(+0)
$$

where $A_{k}, B_{k}$ are constants depending only $u$ pon the boundary conditions, $k=0,1$.

For specific formulas concerning $A_{k}, B_{k}$, we refer to a paper of Birkhoff.*

In order to bring the discussion of the adjoint series of order $n=2 \mu$ under that already carried out, we employ the device of writing $\bar{x}=1-x$, $\bar{y}=1-y, \bar{f}(\bar{y})=f(1-y)$, whereby

$$
\int_{0}^{1} f(y) \int_{C} G(y, x ; \lambda) d \lambda d y=\int_{0}^{1} \bar{f}(\bar{y}) \int_{C} G(1-\bar{y}, 1-\bar{x} ; \lambda) d \lambda d y .
$$

Then on $S^{\prime}$ we find for $n \rho^{n-1} G\left(1-\bar{y}, 1-\bar{x} ; \rho^{n}\right)$ an asymptotic form which in its essentials resembles that for $n \rho^{n} G\left(\bar{x}, \bar{y} ; \rho^{n}\right)$. We are then able to prove Theorems XXIII', XXIV', XXV', XXVI', XXVII', differing from the corresponding theorems of $\S \mathrm{V}$ only in having $n=2 \mu-1$ replaced by $n=2 \mu$.

The theorems on convergence at the end points $x=0, x=1$ present certain differences. We define $\mathfrak{D}^{0}$ and $\mathfrak{D}^{1}$ from $\mathcal{D}(y, 0, \rho)$ and $\mathcal{D}(y, 1, \rho)$ by replacing by zero certain terms of the last columns, as in the corresponding situation in $\S \mathrm{V}$. Then we have

ThEOREM XXVIII'. If $f(x)$ is summable on $(0,1)$, then

$$
\begin{aligned}
\lim _{R \rightarrow \infty} \int_{0}^{1} f(y) \int_{\gamma}\left(n \rho^{n-1} G\left(y, x ; \rho^{n}\right)\right. & -\left\{\sum_{i=\mu+1}^{i=n} e^{\rho \omega_{i}(y-x)} \omega_{i} ;-\sum_{i=1}^{i=\mu} e^{\rho \omega_{i}(y-x)} \omega_{i}\right\} \\
& \left.-\frac{1}{\theta_{2}} \mathcal{D}(y, x, \rho)\right) d \rho d y=0
\end{aligned}
$$

uniformly, $0 \leqq x \leqq 1$.

* Birkhoff, Rendiconti del Circolo Matematico di Palermo, vol. 36 (1913), pp. 125-26. 
ThEOREM XXIX'. If $f(x)$ is summable on $(0,1)$, then the limit in Theorem $\mathrm{XXVIII}^{\prime}$ is valid if $x$ is set equal to 0 or 1 and $\mathcal{D}$ is replaced by the corresponding determinant $\mathfrak{D}$; for example,

$$
\lim _{R \rightarrow \infty} \int_{0}^{1} f(y) \int_{\gamma}\left(n \rho^{n-1} G\left(y, 0 ; \rho^{n}\right)+\sum_{i=1}^{i-\mu} e^{\rho \omega_{i} y} \omega_{i}-\frac{1}{\theta_{2}} D^{0}(y, \rho)\right) d \rho d y=0 .
$$

Theorem $\mathrm{XXX}^{\prime}$ is the exact analogue of Theorem $\mathrm{XXX}$.

TheOREM XXXI'. If $\phi(x)$ is summable on $(0,1)$, and if

$$
\Phi_{1}(x)=\frac{1}{x} \int_{0}^{x} \phi(x) d x, \quad \Phi_{2}(x)=\frac{1}{x} \int_{0}^{x} \phi(1-x) d x
$$

are of bounded variation near $x=0$, then

$$
\lim _{R \rightarrow \infty} \frac{1}{2 \pi i} \int_{0}^{1} \phi(y) \int_{\gamma} n \rho^{n-1} G\left(y, k ; \rho^{n}\right) d \rho d y=\mathfrak{A}_{k} \Phi_{1}(+0)+\mathfrak{B}_{k} \Phi_{2}(+0)
$$

where $\mathfrak{P}_{k}$ and $\mathfrak{B}_{k}$ are constants depending only upon the boundary conditions, $k=0,1$. For example,

$$
+\frac{1}{2 n \theta_{2}}\left|\begin{array}{ccccccc}
a_{1} & \cdots & a_{\mu} & a_{\mu+1} & \cdots & a_{n} & 0 \\
\alpha_{1} \omega_{1}^{k_{1}} & \cdots & \alpha_{1} \omega_{\mu}{ }^{k_{1}} & \beta_{1} \omega_{\mu+1}^{k_{1}} & \cdots & \beta_{1} \omega_{n}^{k_{1}} & -\beta_{1} \sum_{i=1}^{i-\mu} \omega_{i}^{k_{1}+1} \\
\cdot & \cdots & \cdot & \cdot & \cdots & . & \cdot \\
\alpha_{n} \omega_{1}{ }^{k_{n}} & \cdots & \alpha_{n} \omega_{\mu}^{k_{n}} & \beta_{n} \omega_{\mu+1}^{k_{n}} & \cdots & \beta_{n} \omega_{n}^{k_{n}} & -\beta_{n} \sum_{i=1}^{i=\mu} \omega_{i}^{k_{n+1}}
\end{array}\right|,
$$

where $a_{i}=-\Phi_{1}(+0) / \omega_{i}, i=1,2, \cdots, \mu$, and $a_{i}=\Phi_{2}(+0) / \omega_{i}, i=\mu+1, \cdots, n$.

Lastly, we pass to the consideration of the term-by-term derived series,

$$
\frac{1}{2 \pi i} \frac{\partial^{k}}{\partial x^{k}} \int_{0}^{1} f(y) \int_{C_{\nu}} G(x, y ; \lambda) d \lambda d y \quad(k=1,2, \cdots),
$$

under the assumption that the coefficients $p_{2}, \cdots, p_{n}$ of the differential equation are continuous together with their derivatives of all orders. Our theorems deal with the method of summing the series given by the expression

$$
\frac{1}{2 \pi i} \frac{\partial^{k}}{\partial x^{k}} \int_{0}^{1} f(y) \int_{C_{\nu}}\left(1-\frac{\lambda^{4}}{\Lambda_{\nu}^{4}}\right)^{k+l} G(x, y ; \lambda) d \lambda d y, \quad l \geqq 0 \text { 。 }
$$


Lemmas XIII and XIV hold for $n=2 \mu$ as well as for $n=2 \mu-1$. If we write

$$
\begin{aligned}
& F_{k}^{0}(x, y, \rho) \equiv-\sum_{i=1}^{i=\mu} \sum_{s=0}^{s=k}\left(\rho \omega_{i}\right)^{s} \omega_{i} e^{\rho \omega_{i}(x-y)} \sum_{\alpha+\beta=k-s} A_{\alpha k}(x) B_{\beta}(y), \\
& F_{k}^{1}(x, y, \rho) \equiv+\sum_{i=\mu+1}^{i=n} \sum_{i=0}^{s=k}\left(\rho \omega_{i}\right)^{s} \omega_{i} e^{\rho \omega_{i}(x-y)} \sum_{\alpha+\beta-k=i} A_{\alpha k}(x) B_{\beta}(y),
\end{aligned}
$$

we find

$$
\begin{aligned}
n \rho^{n-1}\left\{\frac{\partial^{k}}{\partial x^{k}} G ; \frac{\partial^{k}}{\partial x^{k}} G\right\} \equiv\left\{F_{k}^{0}-\sum_{i=1}^{i=\mu} e^{\rho \omega_{i}(x-y)} \frac{m_{i}}{\rho} ; F_{k}{ }^{1}+\sum_{i=\mu+1}^{i=\mu} e^{\rho \omega_{i}(x-y)} \frac{m_{i}}{\rho}\right\} \\
+\frac{\Delta_{3}^{(k)}}{\left[\theta_{1}\right] e^{2 \rho \omega_{\mu}}+\left[\theta_{0}\right] e^{\rho \omega_{\mu}}+\left[\theta_{2}\right]}
\end{aligned}
$$

where $\Delta_{3}^{(k)}$ is a determinant differing from $\Delta_{3}$ only in having the elements of the first row given by

$$
\begin{aligned}
a_{0 j} & =\left(\rho \omega_{j}\right)^{k} e^{\rho \omega j x}[1] & (j=1, \cdots, \mu) ; \\
a_{0 i} & =\left(\rho \omega_{i}\right)^{k} e^{\rho \omega j(x-1)}[1] & (j=\mu+1, \cdots, n) ; \\
a_{0, n+1} & =0, &
\end{aligned}
$$

for $\rho$ on $S^{\prime}$. The demonstration of Theorem $\mathrm{XXXII}^{\prime}$ then follows the lines of that of Theorem XXXII.

TheOREM XXXII'. If $f(x)$ is summable on $(0,1)$ and if $G(x, y ; \lambda)$ is the Green's function associated with the regular differential system of order $n=2 \mu$ of the type described above; and if $F_{k}{ }^{0}(x, y, \rho), F_{k}^{1}(x, y, \rho)$ are defined by the identities above, then

$$
\lim _{R \rightarrow \infty} \int_{0}^{1} f(y) \int_{\gamma}\left(1-\frac{\rho^{4 n}}{R^{4 n}}\right)^{k+l}\left(n \rho^{n-1}\left\{\frac{\partial^{k} G}{\partial x^{k}} ; \frac{\partial^{k} G}{\partial x^{k}}\right\}-\left\{F_{k}^{0} ; F_{k}^{1}\right\}\right) d \rho d y=0
$$

uniformly, $0<a \leqq x \leqq b<1$, for $l \geqq 0$. The expression

$$
\frac{1}{2 \pi i} \frac{\partial^{k}}{\partial x^{k}} \int_{0}^{1} f(y) \int_{C_{\nu}}\left(1-\frac{\lambda^{4}}{\Lambda_{p}^{4}}\right)^{k+l} G(x, y ; \lambda) d \lambda d y
$$

is therefore equivalent on any interval $(a, b)$ completely interior to $(0,1)$ to a linear combination with coefficients $A_{\alpha k}(x)$ of means of order $k+l, l \geqq 0$, formed from the Fourier series and their derived series up to order $k$ for the functions $f(x) B_{0}(x) \equiv f(x), f(x) B_{1}(x), \cdots, f(x) B_{k}(x)$. On any interval $(a, b)$ the problem of derived Burkhoff series of order $n=2 \mu$ is reduced to a problem in Fourier series and their derived series. 
Theorems XXXIV' and XXXVI', analogous to the correspondingly numbered theorems of $\S \mathrm{VI}$, can then be obtained without difficulty.

From our brief indications the reader will perceive that the case $n=2 \mu$ is in its main features similar to the case $n=2 \mu-1$, which we have discussed in greater detail.

Colombia UNIVERSITY, NEW YoRK, N.Y. 